University of Tennessee Health Science Center

UTHSC Digital Commons

\title{
Effect of Obesity on the Traditional and Emerging Cardiovascular Disease Risk Factors in African American Women
}

Queen Obiageli Henry-Okafor

University of Tennessee Health Science Center

Follow this and additional works at: https://dc.uthsc.edu/dissertations

Part of the Cardiovascular Diseases Commons, and the Women's Health Commons

\section{Recommended Citation}

Henry-Okafor, Queen Obiageli , "Effect of Obesity on the Traditional and Emerging Cardiovascular Disease Risk Factors in African American Women" (2009). Theses and Dissertations (ETD). Paper 341. http://dx.doi.org/10.21007/etd.cghs.2009.0133. 


\title{
Effect of Obesity on the Traditional and Emerging Cardiovascular Disease Risk Factors in African American Women
}

\begin{abstract}
Background: Obesity is a growing health care concern with cardiovascular disease (CVD) implications. African American women (AAW) have the highest prevalence rate of obesity and highest CVD morbidity and mortality rate of all ethnic groups. The traditional CVD risk factors have not been sufficient to explain this disparity in disease prevalence and outcomes. Current knowledge is limited regarding the interaction between various levels of adiposity and both traditional and emerging CVD risk factors, particularly in AAW. This study sought to explore these interactions.

Methods: The study design was a cross-sectional, descriptive, correlational analysis of 48 AAW ages 18 to 45 who had no known history of CVD. Participants completed a demographic/health history questionnaire and 7-Day Physical Activity Recall Questionnaire. Anthropometric assessment of height, weight, BMI, waist circumference, and waist-hip-ratio were determined. An average of two resting blood pressures was taken. Hypertension was defined as a systolic blood pressure $\geq 140 \mathrm{mmHg}$ or diastolic blood pressure $\geq 90 \mathrm{mmHg}$. Fasting blood levels were drawn of traditional (glucose, total cholesterol, low density lipoprotein cholesterol, high density lipoprotein cholesterol, triglycerides) and emerging risk factors (fibrinogen, high density C-reactive protein [hs-CRP], plasminogen activator inhibitor-1 [PAI-1], Eselectin, quantitative insulin sensitivity check index [QUICKI], and soluble intercellular adhesion molecule-1 [sICAM-1]). Prediabetes and diabetes were determined based on the American Diabetes Association criteria. Normal values for lipids were based on Adult Treatment Panel (ATP) III guidelines. Reference lab norms were used for fibrinogen ( $<350 \mathrm{mg} / \mathrm{L})$, hs-CRP $(<2 \mathrm{mg} / \mathrm{L})$, and PAl- $1(<28 \mathrm{mg} / \mathrm{mL})$. Normative values for QUICKI, sICAM-1, and E-selectin have not yet been established, thus cut-points used for these tests were from previous research studies. Analyses included descriptive statistics, t-test, chisquare, and Spearman's correlation analysis. An alpha level of 0.05 was set a priori for statistical significance.
\end{abstract}

Results: Only $4.17 \%$ of our participants reported a history of diabetes and hypertension. The most common traditional risk factor identified was physical inactivity $(72.92 \%)$, followed by a positive family history of CVD (58.3\%). Obesity, defined as a measured BMI $\geq 30 \mathrm{~kg} / \mathrm{m}^{2}$, was present in $56.25 \%$. All of our participants had insulin resistance, and $56.25 \%$ had elevated fibrinogen levels. Our findings indicated that the obese weight group had a higher systolic blood pressure $(p=0.0002)$ and diastolic blood pressure $(p$ $=0.0007)$, and lower HDL-c $(p=0.01)$ and higher triglyceride levels $(p=0.02)$ than the normal weight group. The obese group had significantly higher levels of hs-CRP $(p=0.002)$ and fibrinogen $(p=0.01)$ compared to the normal weight groups. Compared to the normal weight group, the obese group had significantly more CVD risk factors (6.9 vs. $4.1, \mathrm{p}<0.05)$.

Conclusion: Obesity is associated with a higher prevalence of both the emerging and traditional cardiovascular risk factors in AAW without a previous history of CVD. Physical inactivity is the most prevalent modifiable risk factor in this population. Focused intervention on obesity and physical inactivity could provide substantial reduction in CVD morbidity and mortality among AAW

\author{
Document Type \\ Dissertation \\ Degree Name \\ Doctor of Philosophy (PhD)
}




\section{Program}

Nursing

Research Advisor

Patricia A. Cowan, Ph.D.

\section{Keywords}

African American Women, Cardiovascular Disease, Obesity, Physical Activity, Prevalence, Risk Factors

\section{Subject Categories}

Cardiovascular Diseases | Diseases | Medicine and Health Sciences | Women's Health 


\title{
EFFECT OF OBESITY ON THE TRADITIONAL AND EMERGING CARDIOVASCULAR DISEASE RISK FACTORS IN AFRICAN AMERICAN WOMEN
}

\author{
A Dissertation \\ Presented for \\ The Graduate Studies Council \\ The University of Tennessee \\ Health Science Center
}

\begin{abstract}
In Partial Fulfillment
Of the Requirements for the Degree

Doctor of Philosophy

From The University of Tennessee
\end{abstract}

By

Queen Obiageli Henry-Okafor

May 2009 
Copyright (C) 2009 by Queen Henry-Okafor All rights reserved 


\section{DEDICATION}

This dissertation is dedicated to the Almighty God who has done more than I could ask or imagine (Ephesians 3:20).

And to my dear husband, Dr. Henry E. Okafor, without whose support this endeavor could not have been accomplished. And to the greatest gift of all, our son, Ebenezer Akachukwu Henry-Okafor, who showed much grace all through this process;

I remain greatly indebted. 


\section{ACKNOWLEDGEMENTS}

I would like to express heartfelt gratitude to my dissertation chair, Dr. Patricia Cowan, for her support, encouragement, and guidance. I would also like to thank my dissertation committee members, Drs. Mona Wicks, Muriel Rice, Sharon Husch, and Michelle Khoo for their support and insightful contributions to my dissertation.

Additionally, I would like to thank my husband Dr. Henry E. Okafor for his insight and invaluable support throughout this project. I would also like to thank my family members (Joan, Comfort, Kingsley, Chukwuemeka, Uche) for their continued love and encouragement. Special thanks to my parents (late Stephen and Eugenia Agomoh) for a firm foundation. Also thanks to Mrs. Stella Nwokeji for her support and assistance with recruitment of subjects for the study. I would like to thank all the beautiful women who participated in my study. Their willingness made this possible.

I would also like to acknowledge the support provided by the Southern Regional Education Board for the dissertation year fellowship award, the University of Tennessee Health Science Center College of Nursing for assisting with laboratory costs, and the College of Nursing and the College of Graduate Health Sciences for travel support to present research findings. 


\begin{abstract}
Background: Obesity is a growing health care concern with cardiovascular disease (CVD) implications. African American women (AAW) have the highest prevalence rate of obesity and highest CVD morbidity and mortality rate of all ethnic groups. The traditional CVD risk factors have not been sufficient to explain this disparity in disease prevalence and outcomes. Current knowledge is limited regarding the interaction between various levels of adiposity and both traditional and emerging CVD risk factors, particularly in AAW. This study sought to explore these interactions.
\end{abstract}

Methods: The study design was a cross-sectional, descriptive, correlational analysis of 48 AAW ages 18 to 45 who had no known history of CVD. Participants completed a demographic/health history questionnaire and 7-Day Physical Activity Recall Questionnaire. Anthropometric assessment of height, weight, BMI, waist circumference, and waist-hip-ratio were determined. An average of two resting blood pressures was taken. Hypertension was defined as a systolic blood pressure $\geq 140 \mathrm{mmHg}$ or diastolic blood pressure $\geq 90 \mathrm{mmHg}$. Fasting blood levels were drawn of traditional (glucose, total cholesterol, low density lipoprotein cholesterol, high density lipoprotein cholesterol, triglycerides) and emerging risk factors (fibrinogen, high density C-reactive protein [hsCRP], plasminogen activator inhibitor-1 [PAI-1], E-selectin, quantitative insulin sensitivity check index [QUICKI], and soluble intercellular adhesion molecule-1 [sICAM-1]). Prediabetes and diabetes were determined based on the American Diabetes Association criteria. Normal values for lipids were based on Adult Treatment Panel (ATP) III guidelines. Reference lab norms were used for fibrinogen $(<350 \mathrm{mg} / \mathrm{L})$, hsCRP $(<2 \mathrm{mg} / \mathrm{L})$, and PAI-1 $(<28 \mathrm{mg} / \mathrm{mL})$. Normative values for QUICKI, sICAM-1, and E-selectin have not yet been established, thus cut-points used for these tests were from previous research studies. Analyses included descriptive statistics, t-test, chi-square, and Spearman's correlation analysis. An alpha level of 0.05 was set a priori for statistical significance.

Results: Only 4.17\% of our participants reported a history of diabetes and hypertension. The most common traditional risk factor identified was physical inactivity $(72.92 \%)$, followed by a positive family history of CVD (58.3\%). Obesity, defined as a measured $\mathrm{BMI} \geq 30 \mathrm{~kg} / \mathrm{m}^{2}$, was present in $56.25 \%$. All of our participants had insulin resistance, and $56.25 \%$ had elevated fibrinogen levels. Our findings indicated that the obese weight group had a higher systolic blood pressure $(\mathrm{p}=0.0002)$ and diastolic blood pressure $(\mathrm{p}=$ $0.0007)$, and lower HDL-c $(p=0.01)$ and higher triglyceride levels $(p=0.02)$ than the normal weight group. The obese group had significantly higher levels of hs-CRP ( $p=$ $0.002)$ and fibrinogen $(p=0.01)$ compared to the normal weight groups. Compared to the normal weight group, the obese group had significantly more CVD risk factors (6.9 vs. $4.1, \mathrm{p}<0.05)$.

Conclusion: Obesity is associated with a higher prevalence of both the emerging and traditional cardiovascular risk factors in AAW without a previous history of CVD. Physical inactivity is the most prevalent modifiable risk factor in this population. Focused 
intervention on obesity and physical inactivity could provide substantial reduction in CVD morbidity and mortality among AAW. 


\section{TABLE OF CONTENTS}

CHAPTER 1. INTRODUCTION .....................................................................................1

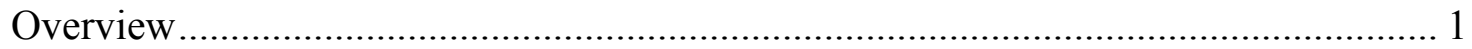

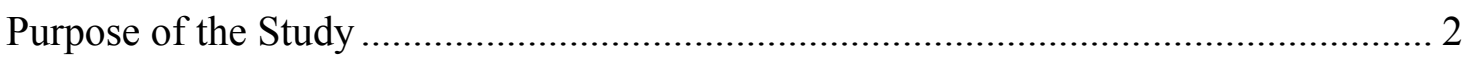

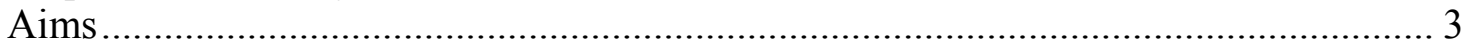

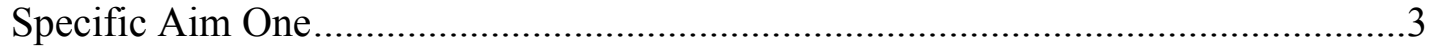

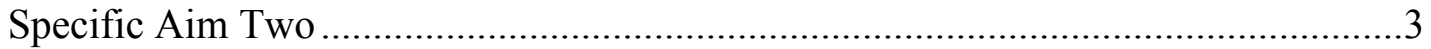





Definitions of Major Concepts......................................................................... 7

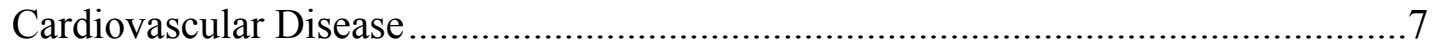

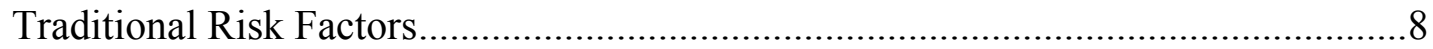

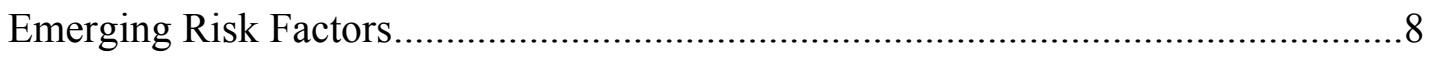

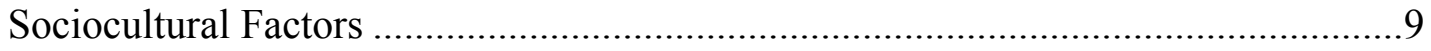

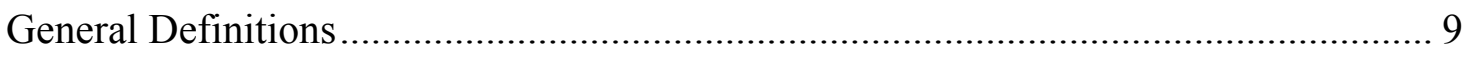

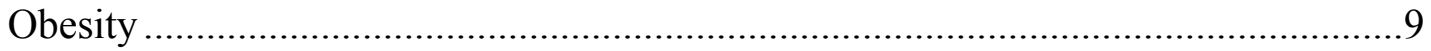

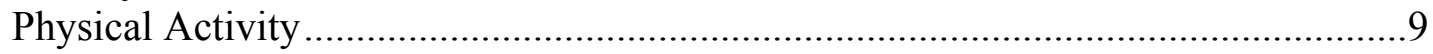

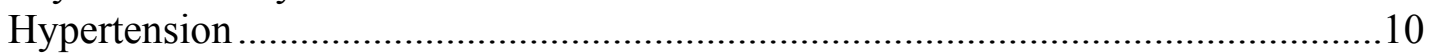

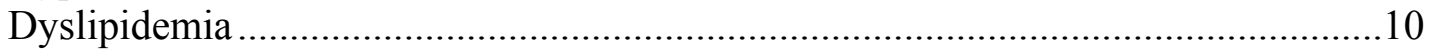

Diabetes Mellitus and Impaired Glucose Tolerance .......................................... 11

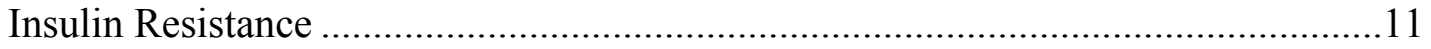

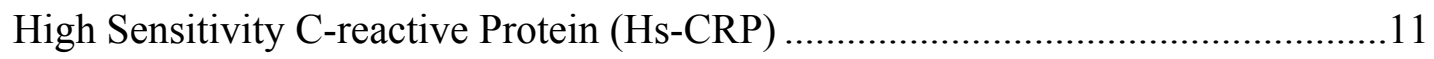

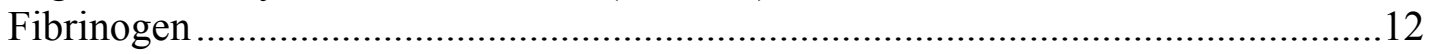

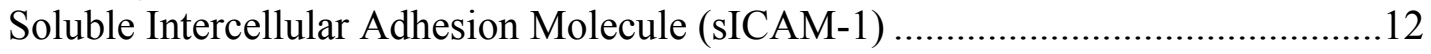

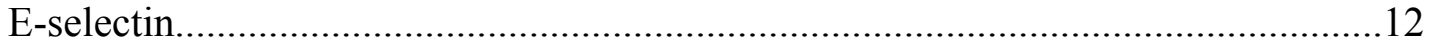

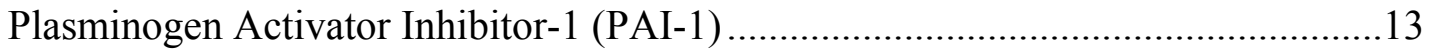

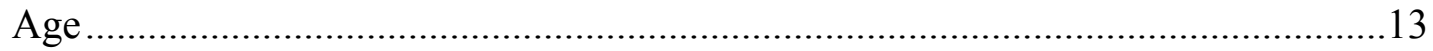

African American Woman...............................................................................13

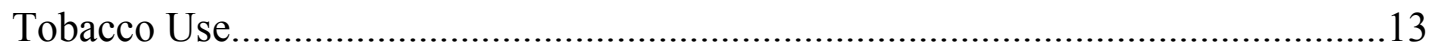

Family History of Cardiovascular Disease .....................................................13

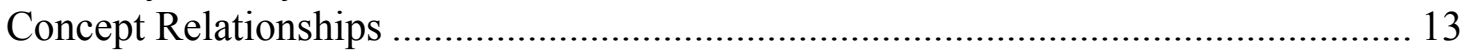

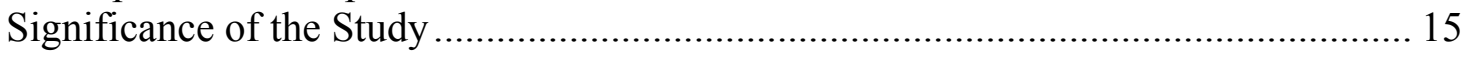

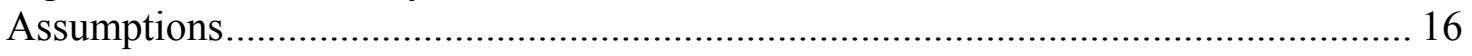

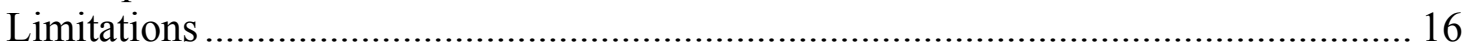

CHAPTER 2. REVIEW OF LITERATURE ...................................................18

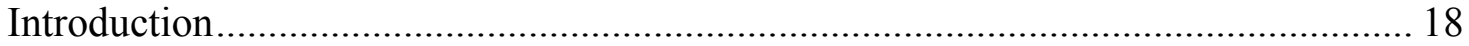



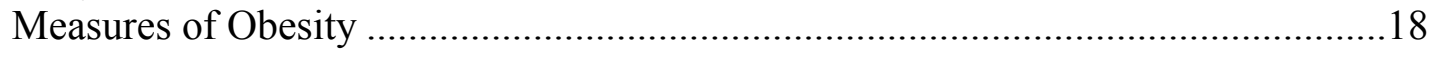

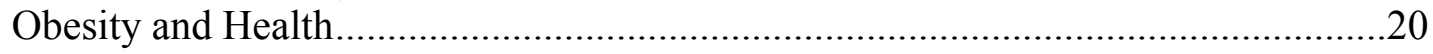

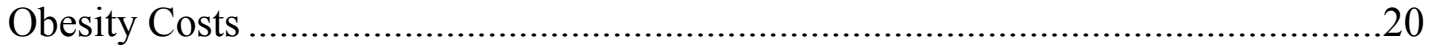

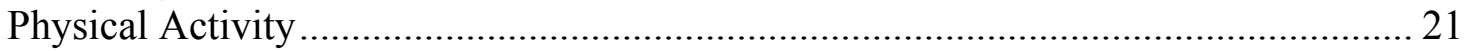

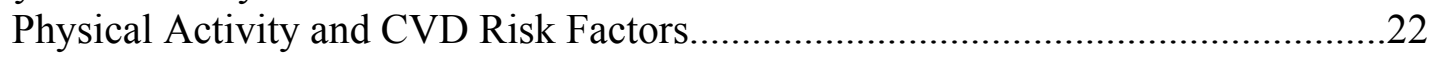

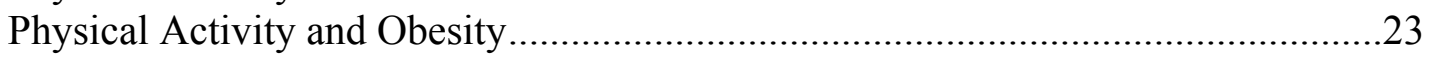


Types of Physical Activity …………………………..........................................23

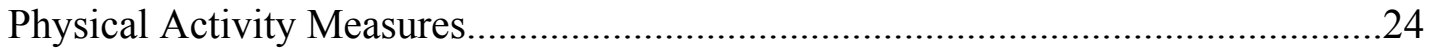

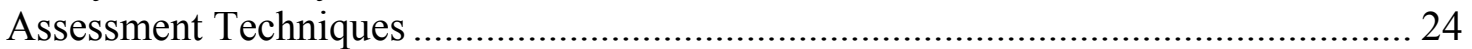



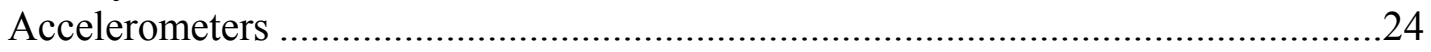



Physical Activity Questionnaires, Recalls, and Records ..........................................25

Seven-Day Physical Activity Recall Questionnaire ..............................................26

Traditional Cardiovascular Disease Risk Factors ……….............................................. 27

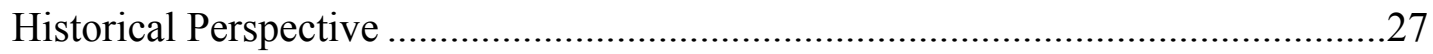

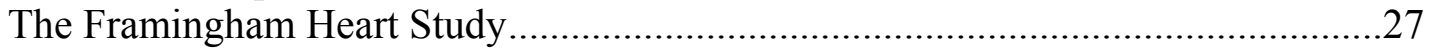

Emerging Cardiovascular Disease Risk Factors ………............................................ 29

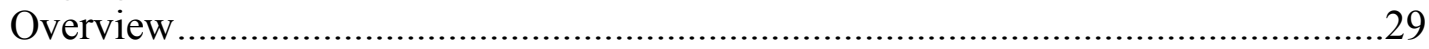

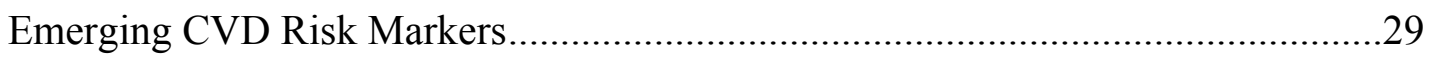

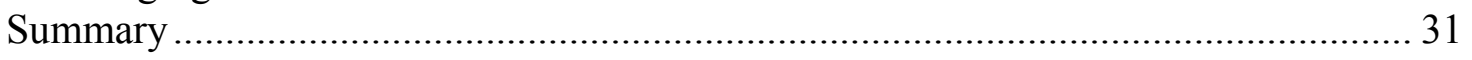

CHAPTER 3. METHODOLOGY ..............................................................................32

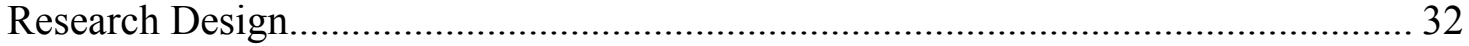

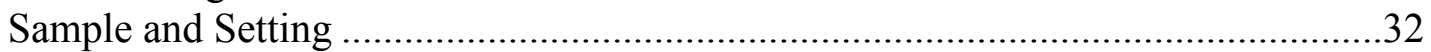

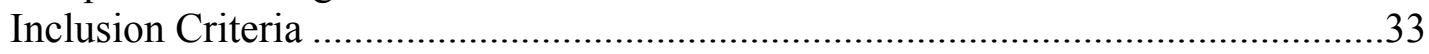

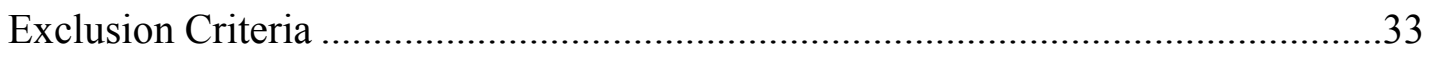

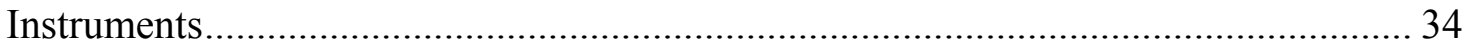



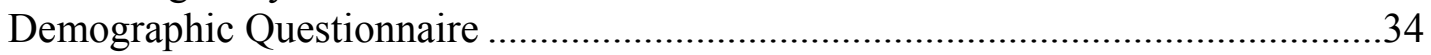

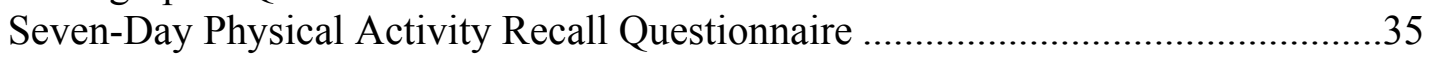

Blood Pressure and Heart Rate Screening .............................................................35

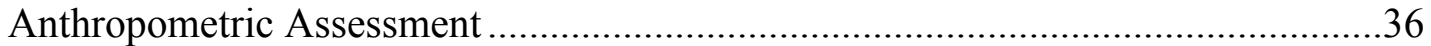

Blood Collection for Traditional and Emerging Risk Factors .....................................36

Traditional Cardiovascular Risk Factors ……………............................................ 37

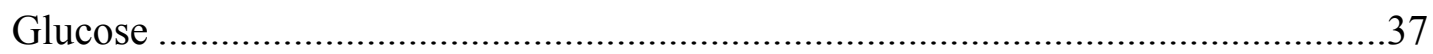



Emerging Cardiovascular Risk Factors Screening ………...................................... 38



High Sensitivity C-reactive Protein (hs-CRP) .........................................................39

Plasminogen Activator Inhibitor-1 (PAI-1), E-selectin, and Soluble ICAM-1 .........39

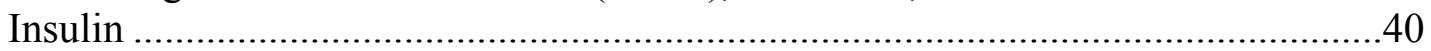

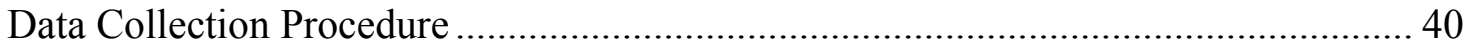

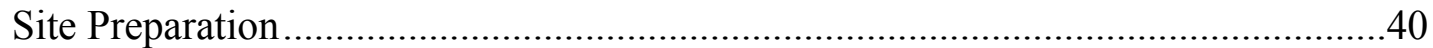

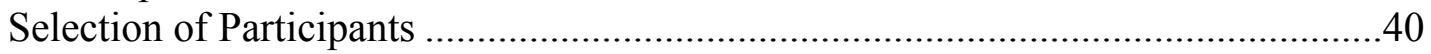

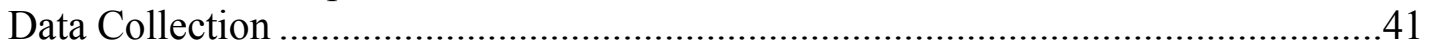

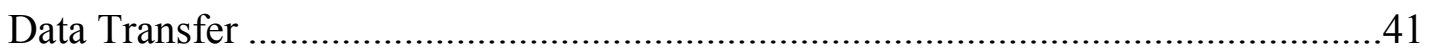

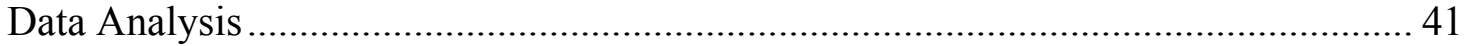

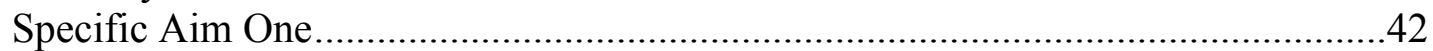

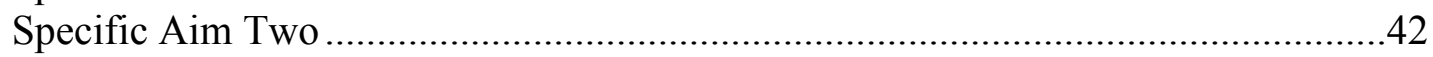

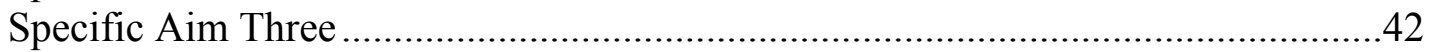

Consideration of Human Subjects ........................................................................ 43 


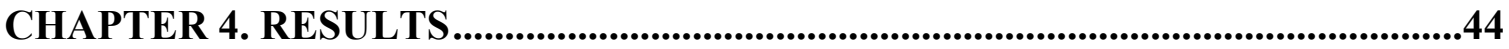

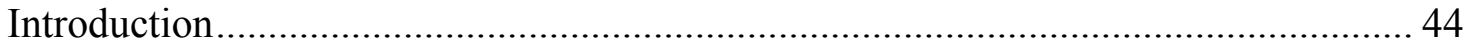

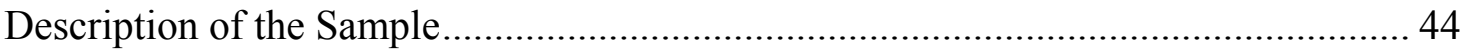

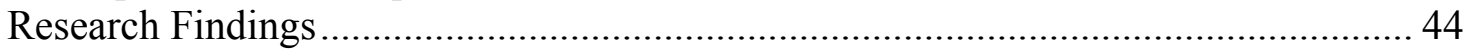



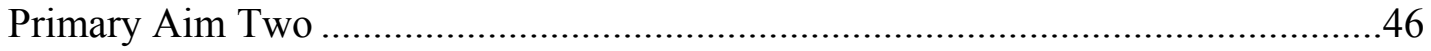

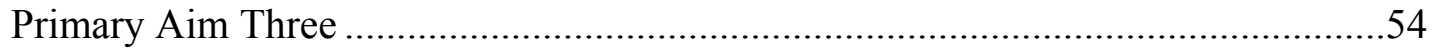

Average Number of Risk Factors by Weight Group ..............................................58

Average Number of Risk Factors by Physical Activity .............................................58

CHAPTER 5. DISCUSSION AND IMPLICATIONS..............................................60

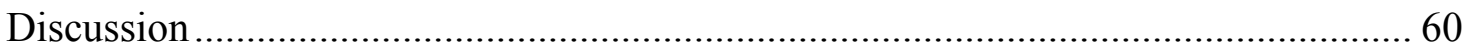

Prevalence of Traditional and Emerging Cardiovascular Risk Factors ......................60

Relationships between Cardiovascular Risk Factors ..................................................62

Relationships between BMI, Physical Activity, and Blood Pressure .........................62

Correlations between BMI, Physical Activity, and Diabetes ......................................62

Relationships between BMI, Physical Activity, and Insulin Resistance ....................63

Correlations between BMI, Physical Activity, and Lipid Levels ................................63

Relationships between BMI, Physical Activity, and Tobacco Use ............................64

Correlations between BMI, Physical Activity, and Family History of CVD .............64

Relationships between BMI, Physical Activity, and Inflammation............................65

Correlations between Obesity, Physical Activity, and Thrombotic and

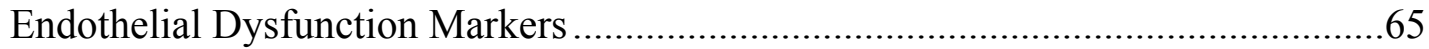

Differences in Cardiovascular Risk Factors by Weight Groups .................................67

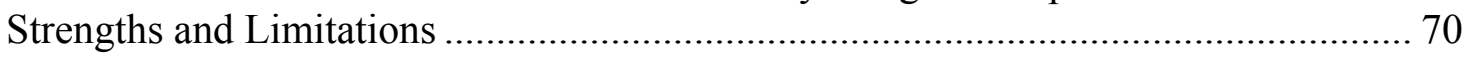

Relation of Study Results to Conceptual Framework ................................................... 71

Practice and Policy Implications......................................................................... 72

Recommendations for Future Research ……………............................................. 72



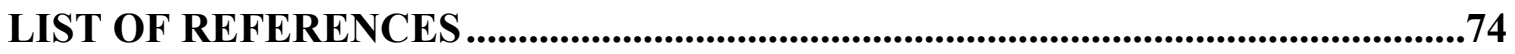

APPENDIX A. DEMOGRAPHIC QUESTIONNAIRE....................................................94

APPENDIX B. 7-DAY PHYSICAL ACTIVITY RECALL QUESTIONNAIRE ......96

APPENDIX C. UNIVERSITY OF TENNESSEE HEALTH SCIENCE

CENTER INSTITUTIONAL REVIEW BOARD APPROVAL ...................................99

APPENDIX D. RECRUITMENT FLIER AND ANNOUNCEMENT .......................100

APPENDIX E. CONSENT FORM..........................................................................102

VITA....................................................................................................................................107 


\section{LIST OF TABLES}

Table 4.1 Subjects' Demographic Characteristics ...............................................45

Table 4.2 Prevalence of Traditional Cardiovascular Disease Risk Factors $(\mathrm{N}=$

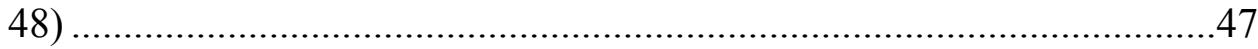

Table 4.3 Prevalence Rates of Emerging Cardiovascular Disease Risk Factors (N



Table 4.4 Descriptive Statistics of Traditional and Emerging Cardiovascular Risk Factors ...............................................................................49

Table 4.5 Relationship among BMI, Physical Activity, and CVD Risk Factors (N $=48)$

Table 4.6 Demographic Characteristics by Weight Groups ..................................55

Table 4.7 Comparison of Means of the Traditional and Emerging Cardiovascular Risk Factors among Normal Weight and Obese African American

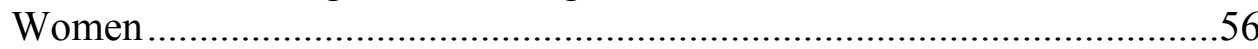

Table 4.8 Comparison of Traditional and Emerging Cardiovascular Risk Factors among Normal Weight and Obese African American Women .....

Table 4.9 Differences in Frequency of Cardiovascular Risk Factors between Weight Groups 


\section{LIST OF ABBREVIATIONS}

$\begin{array}{ll}\text { AAMC } & \text { Association of American Medical Colleges } \\ \text { ACE } & \text { American Council on Exercise } \\ \text { AHA } & \text { American Heart Association } \\ \text { AOA } & \text { American Obesity Association } \\ \text { ARIC } & \text { Atherosclerosis Risk in Communities } \\ \text { ATP } & \text { Adult Treatment Panel } \\ \text { BMI } & \text { Body Mass Index } \\ \text { CARDIA } & \text { Coronary Artery Risk Development in Young Adults } \\ \text { CDC } & \text { Centers for Disease Control and Prevention } \\ \text { CVD } & \text { Cardiovascular Disease } \\ \text { Hs-CRP } & \text { High Sensitivity C-reactive Protein } \\ \text { HTN } & \text { Hypertension } \\ \text { JNC 7 } & \text { Seventh Joint National Committee } \\ \text { JUPITER } & \text { Justification for the Use of Statins in Prevention: An } \\ & \text { Intervention Trial Evaluating Rosuvastin } \\ \text { MESA } & \text { Multi-Ethnic Study of Atherosclerosis } \\ \text { MET } & \text { Metabolic Equivalent } \\ \text { MONICA } & \text { Monitoring Trends and Determinants in } \\ \text { NCEP } & \text { Cardiovascular Disease } \\ \text { PAI-1 } & \text { National Cholesterol Education Program } \\ \text { QUICKI } & \text { Plasminogen Activator Inhibitor-1 } \\ \text { SICAM-1 } & \text { Quantitative Insulin Sensitivity Check Index } \\ \text { WC } & \text { Soluble Intercellular Adhesion Molecule-1 } \\ \text { WHO } & \text { Waist Circumference } \\ \text { WHR } & \text { World Health Organization } \\ & \text { Waist-Hip-Ratio } \\ \end{array}$




\section{CHAPTER 1. INTRODUCTION}

\section{Overview}

Obesity affects a large proportion of the United States population and has become a significant public health problem. Among the United States adult population aged 20 years or older, the prevalence of obesity doubled in the last two decades with over one third of women being obese (American Obesity Association [AOA], 2002). African American women have the highest rate of obesity compared with other women in the United States with more than $50 \%$ of them disproportionately affected (Flegal, Carroll, Ogden, \& Johnson, 2002). Additionally, at similar levels of adiposity, African American women have more obesity related diseases compared to other ethnic minorities (Ogden et al., 2006; Poirier \& Eckel, 2002; Rao, Donahue, Pi-Sunyer, \& Fuster, 2001). This increasing obesity prevalence raises concern because of its public health implications.

Obesity is a major cause of preventable deaths in the United States as well as a potent risk factor for cardiovascular disease with atherosclerotic cardiovascular disease as its foremost physical consequence (Grundy, Hansen, Smith, Cleeman, \& Kahn, 2004). Obesity affects cardiovascular disease morbidity and mortality mainly by promoting the occurrence of cardiovascular disease risk factors such as insulin resistance, type 2 diabetes, dyslipidemia, hypertension, and reportedly has an impact on markers of inflammation, thrombosis, and endothelial function (Poirier \& Eckel, 2002).

Racial and ethnic minorities in the United States have a risk of cardiovascular disease that is at least equivalent to that of the Caucasian majority (Ferdinand, 2006). African Americans, especially women, have the highest overall cardiovascular disease mortality of any ethnic group in the United States (Ford, Giles, \& Dietz, 2002).

According to the American Obesity Association (2002), African American women have a higher cardiovascular disease prevalence rate (44.7\%) compared to Caucasian women (32.4\%). The reason for this disparity is not well understood. Several risk factors increase the risk of developing cardiovascular disease. One potential reason for the increased cardiovascular disease risk in African American women may be the higher prevalence of obesity which has produced a greater clustering of risk factors known as the metabolic syndrome in addition to inadequate screening and a lack of evidence-based treatment of this group in clinical practice (Ferdinand, 2007; Grundy, Hansen, Smith, Jr., Cleeman, \& Kahn, 2004). This makes obesity prevention and management a major public health concern within this population.

The Framingham Heart Study was the landmark study that identified the currently accepted risk factors for cardiovascular disease which includes: hypertension, diabetes, hyperlipidemia, advancing age, gender, physical inactivity, obesity, and a family history of heart disease (Dawber, Meadors, \& Moore Jr., 1951). Even though these traditional risk factors have served some useful purpose in cardiovascular risk stratification, it is debatable how generalizable the findings from this study are given the lack of heterogeneity of the study sample. To bridge this gap, recent clinical studies are 
beginning to include women; notwithstanding, African American women remain grossly underrepresented in most clinical trials.

It is therefore not surprising that despite improvements in cardiovascular risk identification, diagnosis, and management, cardiovascular disease remains the leading cause of death among women in the United States, with African American women excessively affected. It has been previously accepted that cardiovascular disease occurs as a result of lipid abnormalities (Goff, Labarthe, Howard, \& Russell, 2002; Haffner \& Taegtmeyer, 2003; Ross, 1999). An elevated LDL-cholesterol was found in most people with high serum cholesterol mainly because much of the serum cholesterol is transported in LDL-cholesterol. As a result of this association, the evidence suggested that high LDLcholesterol concentrations initiate atherogenesis and promote atherosclerosis. Similarly, a high HDL-cholesterol level has been described as cardioprotective (Cossrow \& Faulkner, 2004). African American women have been shown to have low LDL-cholesterol and high HDL-cholesterol concentrations (Ferdinand, 2007; Gardner, Winkleby, \& Fortmann, 2000; Hall et al., 2006), despite the higher incidence of cardiovascular disease among this population. Traditional cardiovascular risk factors alone have not been sufficient to explain the growing rate of cardiovascular disease morbidity and mortality among African American women (Ridker, Stampfer, \& Rifai, 2001; Rifai \& Ridker, 2001).

There is currently a growing body of evidence linking inflammation to atherosclerosis (Danesh et al., 2000; Ross, 1999). Elevation in markers of inflammation, thrombosis, and endothelial dysfunction (such as hs-CRP, PAI-1, sICAM-1, E-selectin, QUICKI, and fibrinogen) is thought to predict cardiovascular disease risk and outcomes in otherwise healthy individuals (Libby \& Ridker, 2004). These markers of inflammation, thrombosis, and endothelial dysfunction, which complement the traditional risk factors, are now considered the emerging cardiovascular disease risk factors. Their utility in clinical practice and implication to African American women's cardiovascular disease risk stratification is still under investigation.

It is now known that cardiovascular disease starts early in the teenage years but often does not manifest until women are in their late forties or in the perimenopausal years (Nguyen \& McLaughlin, 2002). Early detection of the risk markers for cardiovascular disease will no doubt enhance the development of interventions that could prevent progression of the disease in later years. This research study examined the role of obesity on the traditional and emerging cardiovascular disease risk factors in African American women 18 to 45 years of age.

\section{Purpose of the Study}

Obesity has reached epidemic proportions in the United States - affecting approximately 97 million adults (American Heart Association, 2009a). The increased incidence of cardiovascular disease has been linked directly and indirectly to obesity with its attendant multiple co-morbidities. Obesity is a major cause of preventable deaths in the United States and has been classified by the American Heart Association as a major modifiable risk factor for cardiovascular disease. Billions of dollars in health care costs 
have been attributed to the rising incidence of obesity, making obesity a major public health concern (Finkelstein, Fiebelkorn, \& Wang, 2004; Flegal, Carroll, Ogden, \& Johnson, 2002). African American women have the highest incidence of obesity of all women in the United States, and they suffer enormously from the consequences of obesity-associated diseases. Moreover, there is a paucity of knowledge regarding the association between obesity and the emerging cardiovascular disease risk factors in general - and particularly in African American women. This presents an increasing need for researchers to study the impact of obesity on the traditional and emerging cardiovascular disease risk factors in this understudied population.

This study seeks to describe the prevalence of the traditional and emerging cardiovascular disease risk factors in African American women who are 18 to 45 years of age and to examine the relationship between body mass index (BMI) and the traditional (diabetes, dyslipidemia, hypertension, physical inactivity) and emerging (hs-CRP, PAI-1, E-selectin, QUICKI, fibrinogen, sICAM-1) cardiovascular disease risk factors. The principal hypothesis of this study was that higher BMI is associated with higher levels of the traditional and emerging cardiovascular disease risk factors.

\begin{abstract}
Aims
This study was guided by specific aims that were derived from the literature. The following aims were explored in the course of this study:

\section{Specific Aim One}

Describe the prevalence of traditional and emerging cardiovascular disease risk factors in African American women ages 18 to 45 without a known history of cardiovascular disease.
\end{abstract}

\title{
Specific Aim Two
}

Examine the relationship between BMI, physical activity levels, and other traditional (insulin resistance, diabetes, dyslipidemia, hypertension) and emerging (hsCRP, PAI-1, E-selectin, fibrinogen, sICAM-1, insulin resistance [QUICKI]) cardiovascular disease risk factors in African American women.

\section{Specific Aim Three}

Compare traditional and emerging cardiovascular disease risk factors among normal weight and obese African American women. 


\section{Conceptual Framework}

Although several conceptual frameworks have been utilized in the literature to discuss cardio-metabolic issues, the ideas they discussed, though helpful, were not sufficient to guide the methodological decisions and to aid in the interpretation of our study results. A conceptual framework is a drawing that brings together the concepts and/or theories that guide the investigator in the conduct and analysis of a study (LoBiondo-Wood \& Haber, 1990). The conceptual framework in the current study (Figure 1.1) was developed by the investigators and focuses on the risk factors that contribute to cardiovascular health and their interactions with each other.

The Framingham Heart Study and other subsequent clinical studies have identified several modifiable and non-modifiable risk factors that increase the risk of cardiovascular disease. Modifiable risk factors differ from non-modifiable risk factors because they can be prevented, treated, or controlled (American Heart Association [AHA], 2009d). Examples of the modifiable risk factors include tobacco use, high blood cholesterol, high blood pressure, physical inactivity, obesity, and diabetes mellitus; increasing age, male gender, and heredity are some non-modifiable risk factors. Individuals with the non-modifiable risk factors are not only predisposed to cardiovascular disease but also tend to have more abnormal levels of the modifiable traditional risk factors.

Recent literature shows that the emerging cardiovascular disease risk factors independently contribute to cardiovascular disease even in those with normal levels of the traditional risk factors (Ridker, 2008; Ridker et al., 2008). Additionally, several studies have also implicated multiple social and cultural factors such as racism, literacy levels, psychosocial stress, access to health care, socio-economic status, and health belief systems in the higher prevalence of cardiovascular risk factors among minorities and the development of cardiovascular disease (Albert, Glynn, Buring, \& Ridker, 2006; Griffith, Moy, Reischl, \& Dayton, 2006).

Although the prevalence rate of cardiovascular disease risk factors and mortality has declined over the past few decades, cardiovascular disease remains the leading cause of illness, disability, death, and medical costs in the United States (Brownson, Smith, Jorge, Dean, \& DePrima, 1992; Winkleby, Kraemer, Ahn, \& Varady, 1998). The prevalence rates of modifiable cardiovascular disease risk factors such as smoking, physical inactivity, obesity, dyslipidemia, and hypertension vary across population subgroups. Previous knowledge indicated that cardiovascular disease risk factors are highest among males and older individuals. Current literature suggests that younger African American women do have a clustering of cardiovascular disease risk factors (Diez-Roux, 1999) and have been shown to experience a higher prevalence of cardiovascular disease compared to Caucasian women ( $44.75 \%$ vs. $32.4 \%$, respectively). National data indicates that more women die from coronary heart disease than men, with African American women having the highest morbidity and mortality rate (AHA, 2006).

Cardiovascular disease risk factors are more prevalent among African American women. Several studies, such as the Bogalusa Heart Study, the Coronary Artery Risk 


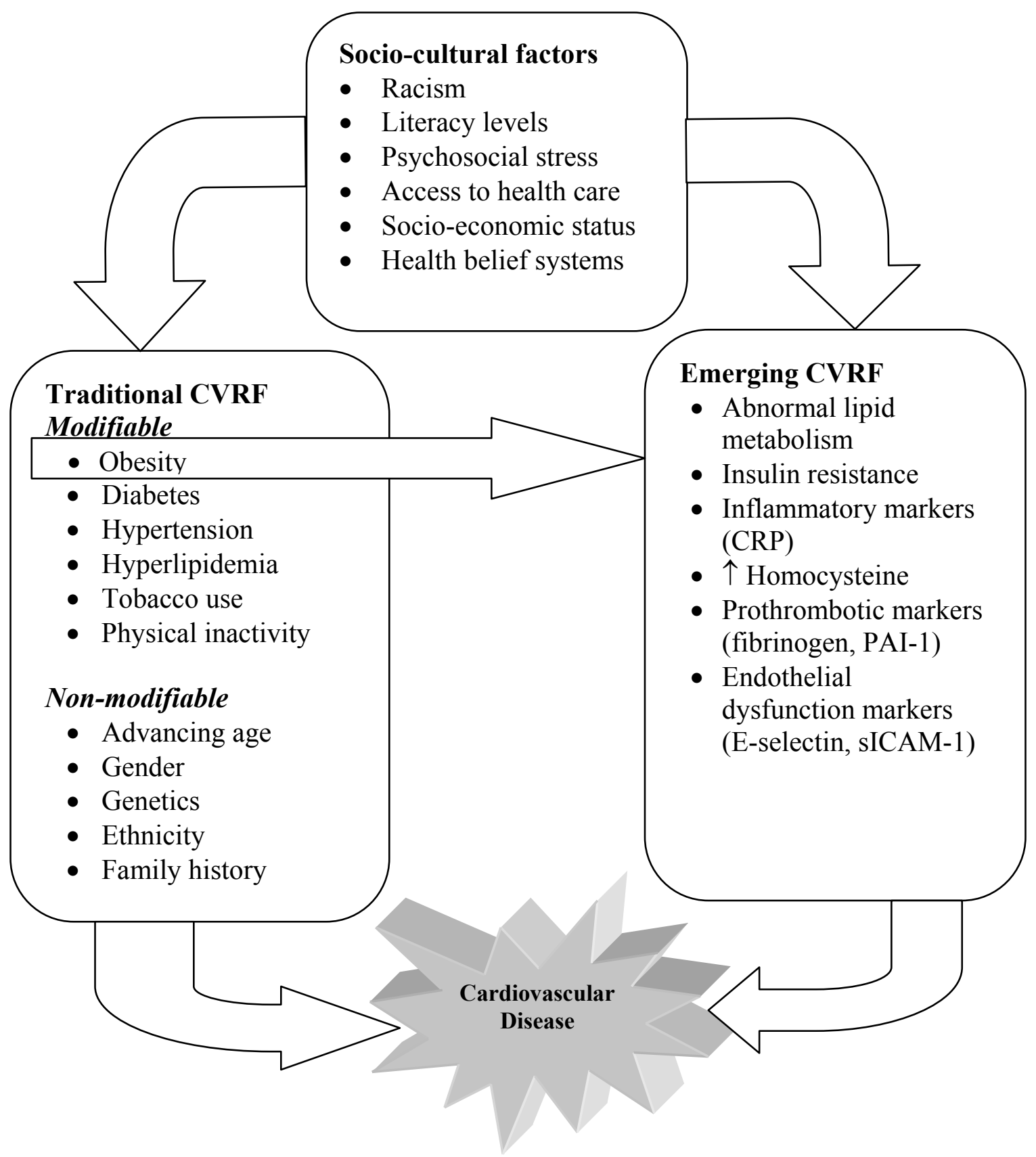

Figure 1.1 Factors Contributing to Cardiovascular Disease

*CVRF $=$ cardiovascular risk factor; $\mathrm{CRP}=\mathrm{C}$-reactive protein; $\mathrm{PAI}-1=$ plasminogen activator inhibitor-1; sICAM-1 = soluble intercellular activator molecule-1 
Development in Young Adults (CARDIA) Study, the Atherosclerosis Risk in Communities (ARIC) Study and the Multi-Ethnic Study of Atherosclerosis (MESA), and most recently the Justification for the Use of Statins in Prevention: An Intervention Trial Evaluating Rosuvastin (JUPITER) trial have all identified cardiovascular disease risk factors in African American women across their lifespan (Adeniyi et al., 2002; Lynch, Liu, Kiefe, \& Greenland, 2006; Nasir et al., 2007; Ridker et al., 2008; Srinivasan, Bao, Wattigney, \& Berenson, 1996). Data from the American Heart Association reveals that $33.9 \%$ of African American women ages 18 and older are physically inactive compared to $21.6 \%$ of white females. Similarly, $77.2 \%$ of African American women ages 20 and older are obese, $41.6 \%$ have cholesterol abnormalities, while an estimated $45.4 \%$ of the women within this age group have high blood pressure. The same data further indicates that $17.2 \%$ of African American women who are 18 years of age and older smoke cigarettes further putting them at risk for cardiovascular disease (AHA, 2006).

Socio-cultural factors such as racism, literacy levels, psychosocial stress, access to health care, socio-economic status, and health belief systems have been reported in the literature as playing a significant role in cardiovascular risk and outcome. Albert, Glynn, Buring, and Ridker's (2006) report indicated that persons of lower socioeconomic status have greater cardiovascular risk than those of higher socioeconomic status. They further noted that educated women were less likely to be smokers, had a lower prevalence of hypertension, diabetes and obesity, and were more likely to participate in vigorous physical activity than less educated women. Similarly, levels of the emerging risk factors such as hs-CRP, sICAM-1, fibrinogen, and homocysteine decreased progressively with increasing education and income categories. Compared to other racial groups, African American women are least aware of their cholesterol status and the least likely to monitor their blood pressure levels (Ayanian, Landon, Landrum, Grana, \& McNeil, 2002). Medical racism both in clinical practice and research has created a perennial distrust of the system that continues to persist till this day (Jacobs, Rolle, Ferrans, Whitaker, \& Warnecke, 2006; LaVeist, Nickerson, \& Bowie, 2000). Despite the clearly positive trends in cardiovascular disease morbidity and mortality, African American women still have higher mortality rates. Socioeconomic status (SES) greatly influences access to health services. Studies reveal that attitudes of young African American women toward food, body image, and physical activity often affect their weight and overall health status (Yanovski et al., 2000). While the validity of such reports are still being debated, there is consensus that the structure of the health system affects screening and treatment of cardiovascular disease risk factors by affecting both clinician and patient behavior (McSweeney, Lefler, Fischer, Naylor Jr., \& Evans, 2007). Persons who are poor, unemployed, have less education, or live in rural or inner-city communities are more likely to experience difficulties obtaining and or affording needed care (Blustein \& Weitzman, 1995). African American females are disproportionately represented among persons of low SES and are more likely to experience problems accessing the health care system (Philbin et al., 2000).

Previous studies have shown a clustering of cardiovascular disease risk factors among poor African Americans. There is a high prevalence of hypertension, physical inactivity, obesity, and smoking among low income African Americans, with education and income level inversely related to hypertension, smoking, physical inactivity, and 
obesity (Diez-Roux, 1999). Similarly, an elevation in some markers of inflammation such as CRP has been reported among socioeconomically disadvantaged persons like African American women (Zhang et al., 2008). Psychosocial work characteristics have been noted to predict cardiovascular disease risk factors and health functioning in women with low SES (Chikani, Reding, Gunderson, \& McCarty, 2004).

Research into emerging cardiovascular disease risk markers such as $\mathrm{C}$-reactive protein, fibrinogen, sICAM-1, PAI-1, and E-selectin is growing, though its influence in clinical practice is still very limited. Homocysteine and abnormal lipid metabolism, while being identified as emerging risk factors, were not studied in the population of this study due to previous studies suggesting that these risk factors were less prevalent in African Americans. Endothelial dysfunction has been identified as an early marker of cardiovascular disease and has been shown to predict future coronary artery disease before atherosclerotic changes appear in arteries. Serum markers of endothelial function such as E-selectin and intercellular adhesion molecule-1 are associated with either increased mortality from or increased prevalence of cardiovascular disease (Abdu, Elhadd, Pfeifer, \& Clayton, 2001) similar to obesity and diabetes. Thrombus formation underpins the development of cardiovascular diseases, including acute coronary syndromes and ischemic stroke. Howes, Keen, Findlay, and Carter (2008) reported that individuals with type 2 diabetes may have a cluster of hemostatic abnormalities including elevated levels of plasminogen activator inhibitor-1 (PAI-1) and fibrinogen that contribute to acute thrombotic events. Elevations in markers of inflammation and thrombosis, such as high sensitivity C-reactive protein (hs-CRP), soluble intercellular adhesion molecule-(sICAM-1), homocystein and fibrinogen, are also associated with increased cardiovascular disease risk. Research has shown that lower levels of physical activity and higher levels of BMI were independently associated ( $\mathrm{P}$ for trend $<.001$ ) with adverse levels of nearly all lipid and inflammatory biomarkers (Mora, Lee, Buring, \& Ridker, 2006).

Thus, socio-cultural factors of racism, low SES, low literacy, limited access to health care, psychosocial stress, and health belief systems provide a milieu conducive to the development of cardiovascular risk factors. The extent of traditional cardiovascular risk factors does not explain the greater cardiovascular disease morbidity and mortality seen in African American women. Within this milieu, these emerging cardiovascular risk factors, regardless of the presence of traditional cardiovascular risk factors, may provide a context for the development of cardiovascular disease in African American women.

\section{Definitions of Major Concepts}

\section{Cardiovascular Disease}

Cardiovascular disease refers to any disease that affects the cardiovascular system. Most of it results from an atherothrombotic pathologic process in the body's arterial beds and is largely preventable through risk factor reduction. Cardiovascular disease is the leading cause of death in women in the United States. Several risk factors 
that have the greatest impact on the incidence of cardiovascular disease in men and women have been identified. Risk factors are diseases, physiologic states, biologic markers, or other identifiable factors associated with increased incidence of cardiovascular disease. These risk factors are categorized as traditional, emerging, and other possible factors that increase the risk for cardiovascular disease.

\section{Traditional Risk Factors}

Traditional cardiovascular disease risk factors are those risk factors that were identified through the Framingham Heart Study. The classic risk factors for cardiovascular disease are hyperlipidemia, diabetes, hypertension, smoking, obesity, physical inactivity, advancing age, male gender, and family history of coronary artery disease. These risk factors are further classified into modifiable and non-modifiable risk factors. The modifiable risk factors refer to those traditional risk factors that can be prevented or treated while the non-modifiable risk factors are nature-imposed. The validity of these risk factors in clinical practice is well established.

\section{Emerging Risk Factors}

Emerging or novel cardiovascular disease risk factors refer to those markers of inflammation, thrombosis, and endothelial dysfunction that could assist in cardiovascular risk stratification for prevention of cardiovascular disease. A growing body of evidence supports recommendations to test asymptomatic individuals deemed to be at risk for cardiovascular disease. Various emerging cardiovascular disease risk factors appear to be important indicators for vascular disease. They include markers of inflammation (high sensitivity C-reactive protein, insulin sensitivity), thrombosis (Plasminogen Activator Inhibitor-1, fibrinogen), and endothelial dysfunction (soluble intercellular adhesion molecule-1, E-selectin). Additionally, insulin resistance, in combination with obesity, has been suggested as the underlying mechanisms for the clustering of cardiovascular disease risk factors that have been identified in the metabolic syndrome

National data indicate that African American women have the highest ageadjusted death rate from cardiovascular disease. The underlying basis for this disparity in cardiovascular disease morbidity and mortality is multifactorial. Although hyperlipidemia screening and treatment has proven to be one of the most effective strategies for reducing cardiovascular disease burden in the U.S. population, it often fails to identify a substantial proportion of persons at high risk for cardiovascular disease-related events. Elevations in markers of inflammation, thrombosis, and endothelial dysfunction are also associated with increased risk. Little data is available relating these emerging risk factors with increased cardiovascular disease risk in racial and ethnic populations. An understanding of the impact of obesity on these emerging risk factors in African American women will enhance our understanding of the disparities in disease prevalence and outcome. 


\section{Sociocultural Factors}

Sociocultural factors are those societal and environmental factors that affect the individual and have far reaching consequences in cardiovascular disease risk factors and cardiovascular disease. They include such factors as racism, socioeconomic status indicators like education and income levels, psychosocial stress, access to health care, and health belief systems.

\section{General Definitions}

\section{Obesity}

Obesity refers to having an abnormally high proportion of body fat —usually $20 \%$ or more over an individual's ideal body weight (National Institutes of Health, National Heart, Lung, and Blood Institute, 1998). The National Institute of Health (NIH) has recommended body mass index (BMI) as a method for determining obesity. BMI correlates with the amount of total body fat and is calculated as weight in kilograms divided by height in meters squared. The Centers for Disease Control and Prevention (CDC, 2007a) and the World Health Organization (WHO, 2004) define obesity in adults as a body mass index (BMI) of $30 \mathrm{~kg} / \mathrm{m}^{2}$ or higher and normal weight as a BMI of 18.5 to $24.9 \mathrm{~kg} / \mathrm{m}^{2}$. This study used BMI to classify participants as obese or normal weight.

\section{Physical Activity}

Physical activity consists of any movement of the body that results in energy expenditure (Caspersen, Powell, \& Christenson, 1985). This study utilized the physical activity recommendations of the U.S. Surgeon General. The recommendation is for people of all ages to engage in a minimum of 30 minutes of moderate physical activity (such as brisk walking) on most, if not all, days of the week (CDC, 1999). Conversely, physical inactivity refers to engaging in less than 30 minutes of moderate or more intense physical activity fewer than 5 days per week. Physical activity levels were quantified using the Seven-Day Physical Activity Recall (7-Day PAR) Questionnaire. The 7-Day PAR Questionnaire is a self-report recall instrument to assess physical activity. Information regarding the duration, intensity, and volume of physical activity engaged in by the individual is used to calculate total daily energy expenditure. For this study, physical activity was defined as total daily energy expenditure as measured by the 7-Day PAR instrument, and physical inactivity was defined as less than 30 minutes of moderate or greater intensity activity on fewer than 5 days per week. 


\section{Hypertension}

Hypertension is often used synonymously with high blood pressure and is a health condition that occurs when there is elevated pressure in the arteries. Hypertension is a silent disorder and requires accurate measurement for diagnosis. Hypertension can be diagnosed using the automatic or auscultatory method of blood pressure measurement with a properly calibrated and validated instrument in a single clinic visit using two readings, 5 minutes apart, sitting in a chair with feet on the floor and arm supported at heart level (Bakris et al., 2007). The terms systolic pressure and diastolic pressure refer to the two numbers that measure blood pressure. Systolic pressure measures cardiac output and refers to the pressure in the arterial system at its highest, while diastolic pressure measures peripheral resistance and refers to arterial pressure at its lowest (Onusko, 2003).

The following categories have been established for blood pressure classification by the Seventh Report of the Joint National Committee on Prevention, Detection, Evaluation, and Treatment of High Blood Pressure (JNC 7, 2003): normal blood pressure is a systolic blood pressure less than $120 \mathrm{mmHg}$ and diastolic blood pressure less than 80 $\mathrm{mmHg}$. The diagnosis of prehypertension is with a systolic blood pressure of 120-139 $\mathrm{mmHg}$ or diastolic blood pressure of $80-90 \mathrm{mmHg}$. An individual is said to have stage 1 hypertension if he/she has a systolic blood pressure of $140-159 \mathrm{mmHg}$ or a diastolic blood pressure of 90-99 mmHg. Stage 2 hypertension occurs with a systolic blood pressure that is $\geq 160 \mathrm{mmHg}$ or a diastolic blood pressure that is $\geq 100 \mathrm{mmHg}$. This study classified blood pressure using the above JNC 7 guideline.

\section{Dyslipidemia}

For the purposes of this study, atherogenic dyslipidemia was based on the standard lipid profile, which includes fasting serum levels of total cholesterol, triglycerides, low-density lipoprotein cholesterol (LDL-c), and high density lipoprotein cholesterol (HDL-c). Normal values for total cholesterol $(<200 \mathrm{mg} / \mathrm{dl}$, LDL-cholesterol $(<130 \mathrm{mg} / \mathrm{dl})$, HDL-cholesterol $(\geq 50 \mathrm{mg} / \mathrm{dl})$, and triglyceride levels $(<150 \mathrm{mg} / \mathrm{dl})$ are based on the criteria established by the National Cholesterol Education Program (Cleeman \& Lenfant, 1998).

The lipid profile or cholesterol tests are performed to reveal information about the types, amount, and distribution of the various types of lipids in the blood stream. An elevated cholesterol level, or excess cholesterol, is deposited on the inside walls of arteries in the form of a plaque. Plaque buildup eventually narrows the arteries causing a condition known as atherosclerosis, which results in cardiovascular disease (Grundy, 1997). Cholesterol and triglycerides levels of study participants were obtained by measuring the following lipoproteins: (a) high density lipoproteins (HDL), which are stable and do not stick to artery walls and (b) low density lipoproteins (LDL), which are unstable and contain more fat and less protein. Unlike HDL, LDL does stick to the cell lining and inside of artery walls. Our study screened for triglycerides, total cholesterol, 
and LDL and HDL cholesterol. Triglyceride is a type of fat in the blood stream; elevated levels are also associated with risk for cardiovascular disease.

\section{Diabetes Mellitus and Impaired Glucose Tolerance}

Diabetes mellitus is a syndrome characterized by disordered metabolism and abnormally high blood sugar resulting from insufficient levels of the hormone insulin (Alberti \& Zimmet, 1998). This study will utilize the fasting plasma glucose level of $\geq$ $126 \mathrm{mg} / \mathrm{dl}$ as the criteria for the diagnosis of diabetes or the prescribed use of antidiabetic therapy in keeping with the Expert Committee on the Diagnosis and Classification of Diabetes Mellitus guidelines. Participants with fasting plasma glucose of 100 to $125 \mathrm{mg} / \mathrm{dl}$ will be categorized as having pre-diabetes consistent with the American Diabetes Association (2007) recommendations.

\section{Insulin Resistance}

Insulin resistance is a component of several health disorders, most notably impaired glucose tolerance and type 2 diabetes mellitus. The cells of insulin resistant individuals are less responsive to the effect of insulin; as a result, higher concentrations of insulin are needed to promote adequate glucose uptake into the insulin-resistant cells (Cefalu, 2001). A significantly reduced level of insulin sensitivity is called insulin resistance. Several methods have been used to evaluate insulin sensitivity. They include: the Euglycemic Hyperinsulinemic Clamp, frequently sampled intravenous glucose tolerance test (FSIGT), Minimal Model Analysis, Oral Glucose Tolerance Test (OGTT), the Homeostasis Model Assessment (HOMA), and the quantitative insulin sensitivity check index model (QUICKI). This study utilized the QUICKI method, which is defined by Katz et al. (2000) with the following formula: $I / \log 1_{0}+\log G_{0}$ where $I_{0}=$ fasting plasma insulin value in $(\mu \mathrm{U} / \mathrm{ml})$ and $\mathrm{G}_{0}=$ fasting plasma glucose value in $(\mathrm{mg} / \mathrm{dl})$. The major advantage of the QUICKI method is that it requires only one blood draw from a fasting individual.

\section{High Sensitivity C-reactive Protein (Hs-CRP)}

C-reactive protein is one of the acute-phase proteins that increase during systemic inflammation and has been suggested as an additional way to assess cardiovascular disease risk (Danesh et al., 2000). High sensitivity CRP (hs-CRP) is a more sensitive CRP test used to determine heart disease risk (American Heart Association, 2009b). HsCRP appears to decrease the synthesis of endothelial nitrous oxide, thus making the endothelium more prone to injury. The following interpretation of hs-CRP results has been recommended by the American Heart Association (2009c): An individual with a hsCRP level $<1.0 \mathrm{mg} / \mathrm{L}$ has a low risk of developing cardiovascular disease, while an individual with a hs-CRP level between 1.0 and $3.0 \mathrm{mg} / \mathrm{L}$ has an average risk of 
developing heart disease. Individuals with a hs-CRP level $>3.0 \mathrm{mg} / \mathrm{L}$ are said to be at high risk for developing cardiovascular disease.

\section{Fibrinogen}

Fibrinogen is an acute phase reactant as well as a plasma protein that plays an important role in thrombosis. Fibrinogen promotes atherosclerosis and reliably predicts prospective risk of initial and recurrent coronary heart disease and stroke (Carty et al., 2008). Fibrinogen degrades into fibrin degradation products, which stimulates smoothmuscle proliferation. Fibrinogen levels appear to be associated with age, body mass, cigarette smoking, diabetes mellitus, total cholesterol, HDL-cholesterol, and triglycerides. Values above $350 \mathrm{mg} / \mathrm{dl}$ were considered elevated (Di Napoli, Papa, \& Bocola, 2001).

\section{Soluble Intercellular Adhesion Molecule (sICAM-1)}

sICAM-1 is a type of intercellular adhesion molecule that presents in low concentrations in the membranes of leukocytes and endothelial cells, a factor crucial to atherosclerosis. ICAM-1 can be induced by interleukin-1 and tumor necrosis factor alpha and is expressed by the vascular endothelium, macrophages, and lymphocytes. ICAM- is either a transmembrane protein (mICAM-1) or soluble (sICAM-1) and has been reported in serum, cerebrospinal fluid, and bronchoalveolar lavage (Wu, McGrath, \& Death, 2005). Circulating sICAM-1 is a marker of cardiovascular disease progression and plays a role in the initiation of atherosclerosis. SICAM-1 predicts the risk for cardiovascular disease or diabetes mellitus in healthy individuals and has been associated with cardiovascular disease death in women (Jenny, Arnold, Kuller, Tracy, \& Psaty, 2007).

\section{E-selectin}

Selectins are a family of cell adhesion molecules. There are three subsets of selectins: E-selectin (in endothelial cells), L-selectin (in leukocytes), and P-selectin (in platelets and endothelial cells). This paper focused on E-selectin, which is a $115 \mathrm{kDa}$, type-1 transmembrane glycoprotein expressed only on endothelial cells and only after activation by inflammatory cytokines (interleukin-1, tumor necrosis factor alpha) or endotoxin (Freestone et al., 2005). Several biological markers have been used as indicators of endothelial dysfunction. Endothelial dysfunction is a key event in cardiovascular disease (Bonora, 2006). Soluble E-selectin is found in the blood of healthy individuals and is increased in individuals with cardiovascular disease and diabetes mellitus. 


\section{Plasminogen Activator Inhibitor-1 (PAI-1)}

Plasminogen activator inhibitor-1 is an important component of the plasminogen/plasmin system, as it is the major physiologic inhibitor of tissue-type and urokinase-type plasminogen activator (Taeye, Smith, \& Vaughan, 2005). PAI-1 is mainly produced by the endothelium (cells lining blood vessels) but is also secreted by other tissue types, such as adipose tissue. Elevations in PAI-1 occur in a variety of clinical situations that are associated with increased risk of ischemic cardiovascular events such as in obesity and metabolic syndrome (Janand-Delenne et al., 1998). PAI-1 has been linked to the increased occurrence of thrombosis in patients and plays an important role in cardiovascular diseases mainly through inhibition of tissue-type plasminogen activator (Gils, 2006). Angiotensin II increases synthesis of PAI-1 and so accelerates the development of atherosclerosis.

\section{Age}

Age was defined as the length of time a person has lived in years. Age was determined by participants' self-report.

\section{African American Woman}

African American woman is a term used to describe females who are citizens or residents of the United States with African ancestry. The participants in this study selfidentified as African American woman.

\section{Tobacco Use}

Tobacco use was defined as self-reported current use of tobacco products including cigarette, cigar, pipe, or spit tobacco.

\section{Family History of Cardiovascular Disease}

The participants in this study self-reported whether a first degree relative had been diagnosed with cardiovascular disease. Specifically, participants identified whether a family member had ever been diagnosed with a heart attack, stroke, or congestive heart failure.

\section{Concept Relationships}

African American women have the highest incidence of cardiovascular disease risk factors such as hypertension, obesity, physical inactivity, smoking, diabetes, and 
dyslipidemia when compared with incidences in the Caucasian and Hispanic populations (Mosca, Ferris, Fabunmi, \& Robertson, 2004). These modifiable risk factors were once thought to be more prevalent in males and aggravated by advancing age. Recent knowledge suggests that these risk factors are equally prevalent in perimenopausal women, although the incidence of coronary heart disease in women increases after menopause (Nguyen \& McLaughlin, 2002). Genetic studies, though evolving, suggest a familial or ethnic inclination to some of these risk factors. Obesity has become a major epidemic in the United States. Previous studies have reported a relationship between obesity and risk factors associated with the development of cardiovascular disease (Folsom et al., 1991). Obese women are four times as likely to develop type 2 diabetes, hyperlipidemia, hypertension, and cardiovascular disease (AOA, 2002) as normal weight women. African American women are twice as likely to be obese as Caucasian women (Flegal et al., 2002). Genetics as a non-modifiable risk factor was not assessed, while age, gender, and ethnicity were controlled for in the study design.

Several factors have been posited in the literature for the increased incidence of the traditional and even the emerging cardiovascular disease risk factors in African American women. African American women are members of an ethnic minority group, which often predisposes them to poor health status, including the risk factors for cardiovascular disease. They often have low income and less education, and studies show that persons of lower socioeconomic status have greater cardiovascular risk than those of higher socioeconomic status (Albert, Glynn, Buring, \& Ridker, 2006). Obesity appears to have a strong inverse relationship with socioeconomic status among women in the United States (AOA, 2002). Although sociocultural factors were not the focus of this study, it is noteworthy that African American women often suffer form psychosocial stress due to longstanding effects of racism and the prevailing lack of access to health care.

Emerging cardiovascular disease risk factors are those markers of inflammation, thrombosis, and endothelial dysfunction that show utility for cardiovascular risk stratification in otherwise healthy persons. Data is limited regarding the impact of these emerging risk factors on cardiovascular health particularly in African American women. Available data show that gender, race, smoking, and BMI were associated with elevated CRP in socioeconomically disadvantaged individuals such as African American women (Zhang et al., 2008).

Hemostatic factors and endothelial markers are said to play some role in racial/ethnic differences in cardiovascular disease rates. Elevated concentrations of haemostatic variables such as fibrinogen and PAI-1 have been found in both overweight and obese African American and Caucasian women (Perry, Wang, Goldberg, Ross, \& Jackson, 2007). African American women have some of the highest levels of fibrinogen and E-selectin but among the lowest levels of intercellular adhesion molecule-1 and PAI1, while Caucasians and Hispanics tend to have intermediate levels of these factors and markers (Lutsey et al., 2006). Similarly, insulin resistance has been associated with increased cardiovascular risk including dyslipidemia, hypertension, and impaired fibrinolysis, particularly in nondiabetic persons. 


\section{Significance of the Study}

Despite growing awareness and availability of intervention efforts, the prevalence of obesity is increasing in virtually all populations and age groups worldwide. This increase is most evident in the United States, with one in four American adults reportedly obese (AOA, 2002). Data from the National Health and Nutrition Examination Survey (NHANES III) 1988-1994 and 1999-2000 reported an increase in the prevalence of obesity across racial and gender groups (National Institutes of Health, National Heart, Lung, and Blood Institute, 1998). Longitudinal data on the development of obesity indicates that the risk of higher obesity rates in women has been greatest in African American women than any other ethnic group (CDC, 2007a). One out of two African American women is obese representing the highest prevalence of obesity compared with Mexican and Caucasian American women (McTigue, Garrett, \& Popkin, 2002).

The annual estimated cost of obesity in the United States is $\$ 117$ billion. This estimate includes $\$ 61$ billion in direct medical costs for treatment of related diseases plus $\$ 56$ billion in indirect costs, such as lost productivity (Finkelstein, Fiebelkorn, \& Wang, 2003). Generally speaking, obese persons incur higher healthcare and medication costs (36\% and $77 \%$, respectively) than normal weight individuals. Emerging evidence about the economic and human costs of obesity has galvanized interest in greater governmental involvement to regulate private behavior in order to promote public health (Mello, Studdert, \& Brennan, 2006). Obesity has become a major focus of public health law because of its growing epidemic and devastating consequences (Nestle \& Jacobson, 2000) which cause more deaths every year than AIDS, all cancers, and all accidents combined.

Several studies have documented the association between obesity and cardiovascular disease. The Framingham data documented that obesity is an independent risk factor for cardiovascular disease for both sexes (Castelli, 1984). Different patterns of obesity appear to correlate to cardiovascular disease incidence. The Nurses' Health Study showed that higher waist/hip ratio and greater waist circumference, measures of abdominal adiposity, were associated with increased risk for cardiovascular disease. Abdominal obesity, when combined with high body mass index, was found to be an important risk factor for cardiovascular disease mortality (Zhang et al., 2008). However, minorities were underrepresented in these studies; thus further work is needed to determine whether these findings can be translated to minority populations.

Current research suggests an association between obesity and the emerging cardiovascular disease risk factors (Prineas, Folsom, \& Kaye, 1993). However, knowledge is limited regarding the impact of obesity on the risk factors for cardiovascular disease in African American women. Consequently, this study examined the association between obesity and the traditional and emerging cardiovascular disease risk factors in self-identified healthy African American women with no clinically apparent cardiovascular disease. 


\section{Assumptions}

The following assumptions were made for this study:

- The study participants honestly and conscientiously completed the demographic and physical activity recall questionnaires.

- There were little or no variability between the various instruments/equipments utilized to obtain subject data for this study.

- Subjects had fasted for 8-10 hours prior to having blood drawn for cardiovascular disease risk markers. Elevations in certain laboratory results, such as lipid profile and insulin and glucose levels, may have occurred if subjects did not adhere to the instructions regarding fasting prior to the blood being drawn.

- Concepts embedded within the conceptual model were relevant for examining the relationship between body weight and cardiovascular disease risk factors in African American women.

\section{Limitations}

There are certain inherent limitations to this pilot study. The convenience sample size was small $(\mathrm{N}=48)$ and subjects were recruited from three cities in the mid-south-a region known to have one of the highest prevalence rates of obesity in the nation. It is unclear that this sample was representative of other African American women in the other regions of the United States and thus findings may not be generalizable.

The 7-Day PAR Questionnaire was utilized to quantify the activity level of participants. This instrument has an inherent recall bias and is subject to errors of recall attributable to memory, omission, and intrusion (Giles-Corti et al., 2006; Richardson, Ainsworth, Jacobs, \& Leon, 2001). Moreover, the utilization of a one-week time frame to evaluate the study participants' physical activity duration, intensity, and volume may not be truly representative of their typical activity patterns.

This study utilized the standard, nationally recognized cut-points to define BMI. The current use of the BMI as the primary index of obesity is not considered optimal, given the fact that it measures only total body weight rather than the amount of fat. There is some concern over the accuracy of the BMI for setting obesity standards since it uses a standard weight against height formula without taking into account whether the weight is fat or muscle (Vazquez, Duval, Jacobs Jr., \& Silventoinen, 2007). Another limitation of the BMI as a method of assessing weight is that it does not account for the individual's body frame, age, gender, race/ethnicity, and nationality. This is because the distribution of fat may have more impact on the individual's cardiovascular risk profile regardless of a "normal" BMI. Likewise, women often have a higher percentage of body fat than men (Gelber et al., 2008). These limitations notwithstanding, there is, however, no better universally agreed index for setting obesity standards and monitoring trends in the 
population at this time other than the BMI. Hence it is used in this study with the understanding of the potential limitations.

Finally, normative values for the emerging risk factors of PAI-1, E-selectin, sICAM-1, and QUICKI have not yet been established. Values used were from previous studies. This limitation can lead to threats to internal validity and could potentially decrease external validity of the study findings. 


\section{CHAPTER 2. REVIEW OF LITERATURE}

\section{Introduction}

Cardiovascular disease morbidity and mortality are higher among African American women compared to Caucasian women, yet awareness of the threat posed by heart disease is low within this population. Lipid abnormality was characterized by the Framingham Heart Study as the main cause of atherosclerotic disease. African American women generally have a favorable lipid profile, implying that there could be other contributing factors as to why they continue to be disproportionately affected by cardiovascular disease compared to all ethnic groups in the United States. Similarly, African American women have higher prevalence rates of high blood pressure, obesity, and physical inactivity than Caucasian women. Obesity is associated with a constellation of risk factors for cardiovascular disease such as hypertension, type 2 diabetes, C-reactive protein, and fibrinogen. Increased physical activity has been shown to ameliorate the levels of these risk factors as well as help to curb the increasing prevalence of cardiovascular disease. This chapter will present a review of relevant literature on the traditional and emerging risk factors for cardiovascular disease.

\section{Obesity}

Several years ago, obesity was viewed mainly as a lifestyle issue but over the past four decades, the United States has witnessed an increase in the prevalence of obesity across every cadre of the population. The problem of obesity is not limited to the United States; the epidemic is evident throughout the Western world. As a result, the World Health Organization (WHO) has declared obesity a major public health concern (WHO, 2004). The Third National Health and Nutrition Examination Survey (NHANES III) tracked obesity rates in the years 1984 to 1992 and 1999 to 2000 and found that obesity rate had grown from $55.9 \%$ to $64.5 \%$. Among United States adults who are 20 years old or more, an estimated $66.3 \%$ are overweight or obese. Women and ethnic minority populations are disproportionately affected by obesity. About $34 \%$ of women in the United States are classified as obese (Ogden et al., 2006), with one in two African American women unduly affected.

\section{Measures of Obesity}

Obesity refers to an excessive amount of body fat in relation to lean body mass. Available literature has described several measures of adiposity which includes: computed tomography $[\mathrm{CT}]$ (measures abdominal fat distribution), magnetic resonance imaging [MRI] (evaluates distribution of abdominal fat), and electrical impedance (quick method of measuring percentage of body fat). These methods are expensive and impractical for routine clinical use. Depending on the purpose, most researchers, endocrinologists and other healthcare professionals utilize the body mass index (BMI), 
waist-hip ratio (WHR), or waist circumference (WC) measures of adiposity (Gelber et al., 2008).

The BMI is widely accepted by the National Institutes of Health (NIH), Centers for Disease Control and Prevention (CDC) and the World Health Organization (WHO) as the primary tool for measuring obesity and is useful for classifying obesity and disease severity. The BMI measures weight in proportion to height and is calculated with the formula: weight in kilograms divided by height in meters squared. Obesity is classified by a BMI of $30 \mathrm{~kg} / \mathrm{m}^{2}$ or above. This refers to a body weight that is at least 30 percent over the ideal weight of a specified height. The BMI was utilized in this study to categorize adiposity levels. Normal or ideal body weight is represented by a BMI of 18.5 $\mathrm{kg} / \mathrm{m}^{2}$ to $24.9 \mathrm{~kg} / \mathrm{m}^{2}$. The BMI as a measure of adiposity has been criticized because it does not take into account the individual's age, gender, body frame, or ethnic origin (Vazquez et al., 2007).

Waist circumference measures abdominal fat and has been associated with increased risk of cardiovascular disease (Lean, Han, \& Morrison, 1995). Cardiometabolic risk clustering sometimes referred to as the metabolic syndrome has been linked to abdominal adiposity. Significant metabolic abnormalities such as insulin resistance, hyperinsulinemia, and elevated triglyceride levels as well as an increased incidence of hypertension, and diabetes have been associated with abdominal obesity. The U.S. Department of Health and Human Services (2000) reported that overweight or obese women who have a waist circumference above 35 inches $(>88 \mathrm{~cm})$ have a high risk for hypertension, type 2 diabetes, and cardiovascular disease. Similar risk applies to men who have a waist circumference measurement above 40 inches $(>102 \mathrm{~cm})$. Similarly, central fat distribution and total body size are equally important determinants of insulin resistance. Sumner et al. (2008) studied the BMI and waist circumference thresholds in African Americans that best predict insulin resistance. They reported that in African Americans, insulin resistance in men was best predicted by a WC $\geq 102 \mathrm{~cm}$ or a BMI $\geq$ $30 \mathrm{~kg} / \mathrm{m}^{2}$ and in women by a $\mathrm{WC} \geq 98 \mathrm{~cm}$ or a BMI $\geq 32 \mathrm{~kg} / \mathrm{m} 2$. Waist circumference measures the distance around the abdomen. Evidence report from the National Heart Lung and Blood Institute [NHLBI] (2006) suggests that WC may be a better health indicator than BMI of $25 \mathrm{~kg} / \mathrm{m}^{2}$ to $34.9 \mathrm{~kg} / \mathrm{m}^{2}$. For individuals with a BMI above 35 $\mathrm{kg} / \mathrm{m}^{2}$, the use of WC for disease risk classification is less pronounced.

Waist-hip-ratio (WHR) is a measure of abdominal adiposity and refers to the ratio of the circumference of the waist to that of the hips. Several studies suggest that abdominal adiposity as measured by WHR is an independent risk factor for cardiovascular disease in men and women (Lapidus et al., 1984; Larsson et al., 1984; Prineas, Folsom, \& Kaye, 1993; Rimm et al., 1995). Individuals with more weight around their waist are at a higher health risk for diabetes and cardiovascular diseases than those with more weight around the hip. Women with a WHR $\geq 0.8$ often referred to as "apple shape," are at an increased health risk of hypertension, diabetes, and cardiovascular (NHLBI). Women with WHR under 0.8, "pear shape," have less risk. Similarly, men with WHR $>1.0$ have a greater risk of these conditions. 
The literature indicates that these three measures are useful in adiposity measurement and health risk stratification, though the BMI has been said to be limited because it does not account for variation in body fat distribution and abdominal fat mass which differ across populations (WHO, 2000). WC closely correlates with BMI and WHR and has been shown to significantly predict risk for cardiovascular disease. Ho et al. (2001) in their study of a Hong Kong population found that BMI and WC proved most effective for men while WC and WHR were preferable for women in predicting cardiovascular disease. There is some debate among researchers regarding which of the measures of central adiposity had a higher health risk predictive value. While some argue for the use of WHR, others prefer the WC as a better measure of central adiposity (Hill et al., 1999; Molarius \& Seidell, 1998). Dalton et al., (2003) evaluated the correlation between WC, WHR, and BMI with cardiovascular disease risk factors in Australian adults. They concluded that any of these three obesity measures adequately predicted cardiovascular disease risk factors but WHR was noted to be the most useful measure of obesity in identifying individuals with cardiovascular disease risk factors.

\section{Obesity and Health}

It is well established that the medical complications of obesity affect nearly every major organ system in the body. Research studies have documented that obesity promotes the occurrence of risk factors for cardiovascular disease such as hypertension, insulin resistance, type 2 diabetes, dyslipidemia, proinflammation and prothrombotic states (Cassano, Rosner, Vokonas, \& Weiss, 1992; Despres et al., 1990). By engendering comorbid conditions, obesity also increases the risk of premature cardiovascular disease morbidity and mortality.

Obesity through its impact on the cardiovascular system has been associated with numerous cardiac complications such as coronary heart disease (CHD), heart failure and sudden death. The American Heart Association (2009c) has described obesity as a major cause of preventable death and an independent risk factor for cardiovascular disease. However, the mechanism for increased cardiovascular risk in obesity is still unclear.

\section{Obesity Costs}

The obesity epidemic has been expensive to manage either by the public or private sectors of the economy. Costs stemming from the management of obesity related diseases have been astronomical. The United States spent about \$75 million dollars in medical costs in 2003 through Medicare and Medicaid benefits for the treatment of obesity related conditions. The price of coping with obesity goes beyond cost of healthcare. Burkhauser \& Cawley (2008) reported that obesity can lead to low earning potential, low workforce participation, unfavorable employment outcomes, and increased utilization of healthcare resources.

Obesity is growing in epidemic proportions in US adults with numerous comorbidities. As a risk factor for cardiovascular disease, obesity is associated with 
numerous cardiac complications through its impact on the cardiovascular system. When adipose tissue accumulates in excess amount, it has implications not only in the individual's health but also weighs heavily on the healthcare costs. Several tools are used to measure adiposity but the BMI in spite of its limitations is a universally accepted measure of adiposity.

\section{Physical Activity}

Physical activity includes anything that makes people move their bodies including household chores, walking, gardening, and dancing (AHA, 2009c). As the prevalence of obesity and overweight in the United States continues to rise, cardiovascular disease consequences are expected to concurrently increase as well. To combat these problems, the United States first launched a national effort in the 1950s to encourage Americans to lead a more active lifestyle. This effort has continued and in the 1970s the emphasis shifted to the cardiovascular benefits of vigorous physical activity such as running, jogging, aerobics, heavy yard work and competitive sport. Physical activity, in contemporary times, joins the front ranks of essential health objectives which include: good nutrition, the use of seat belts, and the prevention of adverse health effects of tobacco (NIH, NHLBI, 1998).

Despite the campaign to engage the public in physical activity, more than $60 \%$ of adults in the United States do not engage in the recommended amount of physical activity, while an estimated $25 \%$ of the adult populations do not engage in any form of physical activity (CDC, 2007b). Furthermore ethnic differences exist in the prevalence of physical inactivity. African American women are less physically active than other population groups (Felton, Boyd, Bartoces, \& Tavakoli, 2002). Fifty-five percent of African American women are physically inactive and perform no leisure time physical activity (U.S. Department of Health and Human Services, 2002). Consequently, African American women have the highest rate of being overweight and obese compared to other groups in the United States with about four out of five African American women classified as either overweight or obese (CDC, 2007b). The lack of routine physical activity among African American women places them at risk for negative health outcomes including type 2 diabetes and cardiovascular disease. Increasing physical activity has become a formidable public health challenge given the need to prevent premature death, unnecessary illness and disability, controlling health care costs, and maintaining a high quality of life with advancing age.

Health benefits appear to be proportional to the amount of activity. Physical activity decreases with age and is less common among women than men and among those with lower income and less education. Furthermore, there are racial and ethnic differences in physical activity rates, particularly among women. According to the U.S. Department of Health and Human Services, in 2007, five southern states ranked the lowest in attaining recommended physical activity rates with African Americans being less likely to achieve physical activity recommendations than Caucasians (CDC, 2008). Additionally, this report indicated that $34.9 \%$ of African Americans in Tennessee were physically inactive compared to $26.3 \%$ of whites. Rates were slightly better in the other 
states (Mississippi: African Americans 26.6\% vs, whites 15.4\%; Alabama: African Americans 21.6\% vs. whites 16.7\%; Louisiana African Americans 29.7\% vs. whites 19.1\%; and North Carolina: African Americans 19.9\% vs. whites 11.3\%). Health consequences of physical inactivity include 13.5 million people with coronary heart disease, 1.5 million suffer from heart attack in a given year, 250,000 suffer from hip fractures each year, over 60 million people (a third of the population) are overweight, and 50 million have high blood pressure.

Given the numerous health benefits of physical activity and the apparent hazards of being inactive, the need for individuals to adopt a physically active lifestyle has been echoed by all healthcare agencies. Physical inactivity is a serious, nationwide problem. Its scope poses a public health challenge for reducing the national burden of unnecessary illness and premature death.

\section{Physical Activity and CVD Risk Factors}

There is increasing evidence linking physical activity to numerous health improvements. A large number of cohort studies have found that physical activity significantly reduces the risk of cardiovascular disease in the United States. Several studies (Blair et al., 1998; Lee, Blair, \& Jackson, 1999; Stevens, Cai, Evenson, \& Thomas, 2002) have evaluated the relationship between physical activity and BMI with the risk of cardiovascular disease and concluded that physical activity had a strong independent association with cardiovascular disease after adjusting for obesity. Blair and colleagues (1998) noted that increased physical activity predicts lower cardiovascular disease risk independent of obesity.

Physical activity also decreases insulin resistance and clinical evidence supports its role in the prevention and management of type 2 diabetes (Knowler et al., 2002; Tuomilehto et al., 2001). Cardiovascular disease, manifesting as coronary artery disease, is the leading cause of morbidity and mortality among patients with type 2 diabetes. There is an inverse relationship between physical activity and insulin resistance which subsequently leads to the development of type 2 diabetes (Eriksson \& Lindgarde, 1996; Helmrich, Ragland, Leung, \& Paffenbarger, 1991). Type 2 diabetes is a costly and serious disease that affects over eight percent of adults in the United States (Erkelens, 2001). Prevalence of type 2 diabetes continues to rise in the United States with 16 million people affected annually. An estimated $11.8 \%$ of African American women over age 20 are afflicted with type 2 diabetes (CDC, 2007a).

Physical activity also reduces the risk of developing hypertension and is equally useful in the management of the disease (Bassett, Fitzhugh, Crespo, King, \& McLaughlin, 2002). Increased physical activity improves endothelial nitric oxide release and insulin mediated vasodilation and substantially lowers elevated blood pressure (Cleland, Petrie, Ueda, Elliott, \& Connell, 1998; Ritz, 2003). Hypertension is an established risk factor for cardiovascular disease (Castelli, 1984), increased physical activity helps lower blood pressure levels thereby decreasing the risk of cardiovascular disease. 
Studies have shown that increased physical activity have profound effects on improving dyslipidemia and other lipid disorders. Clinical studies involving multiethnic groups have found a decrease in LDL-cholesterol, VLDL-cholesterol and triglyceride levels and an increase in HDL-cholesterol levels in individuals who engaged in regular physical activity (Tran \& Weltman, 1985; Wood, Stefanick, Williams, \& Haskell, 1991). The National Cholesterol Education Panel, Adult Treatment Panel III (NCEP ATP III) (2002) has emphasized that physical activity be introduced to hyperlipidemic patients because of its benefits. The purpose of promoting physical activity to patients with hypercholesterolemia is to maintain a healthy body weight, reduce the risk of metabolic syndrome and independently reduce the risk of cardiovascular disease which is prevalent in dyslipidemia given its link to atherosclerosis.

\section{Physical Activity and Obesity}

Physical activity plays an important role in weight loss and weight maintenance. Obesity is recognized as a major public health issue given its rising prevalence and impact on many chronic diseases. Physical inactivity has been blamed for this epidemic. Obesity causes an increased physical strain on the myocardium as well as other comorbid conditions which may contribute to cardiovascular disease. Studies reveal that body weight has a greater influence on diabetes, and physical activity plays a greater role in cardiovascular disease (Chandrashekhar \& Anand, 1991). Simply put, obesity increases the risk of major chronic diseases such as diabetes and cardiovascular disease while physical activity reduces that risk. Physical activity has also been found to be useful in individuals who are physically active and yet obese. A number of studies have evaluated the relative merits of physical activity and obesity (Blair \& Brodney, 1999; Hu et al., 2001). They found that physical activity lowers the risk of being overweight and obese and those who are physically active and are overweight and obese have a lower mortality rate than individuals who were normal weight and sedentary.

Physical activity has several other benefits which include protection against the development of colon cancer, osteoporosis and depression. Generally speaking, physical activity has been shown to improve quality of life by promoting general well being. Despite available information and increased infrastructure, physical activity levels of most Americans remain low. Increased physical activity is directly related to preventing disease, premature death and to maintaining a high quality of life.

\section{Types of Physical Activity}

There is an emerging consensus among epidemiologists, health professionals and experts in exercise science that physical activity need not be vigorous for it to improve health (NIH, NHLBI, 1998). This notion emphasizes the amount rather than the intensity of physical activity making it easier to incorporate into daily lives. Daily activities such as brisk walks, lawn mowing and other household chores that adapt to the individuals lifestyle are sustainable. The U.S. Department of Health and Human Services (CDC, 
2007b) recommendations for physical activity includes moderate-intensity activities of at least 30 minutes per day, 5 or more days per week; or vigorous-intensity activities for at least 20 minutes per day, at least 3 days per week or both. Anything less than these recommended levels of daily physical activity amounts to physical inactivity.

\section{Physical Activity Measures}

The World Health Organization (WHO, 2004) had recommended that energy requirements be based on the assessments of energy expenditure rather than energy intake. The challenge posed by this proposal was due to the difficulty associated with measuring physical activity, a variable component of total energy expenditure, because of the potential to overestimate or underestimate physical activity levels. Several techniques have been developed for use in measuring physical activity such as doubly-labeled water method (DLW), heart rate monitoring, accelerometers, and physical activity questionnaires.

\section{Assessment Techniques}

\section{Doubly-labeled Water}

Doubly-labeled water (DLW) technique was developed in the 1950s by Nathan Lifson (Visser, Boon, \& Meijer, 2000). This method involves taking a load of water labeled with deuterium and oxygen-18 $\left(\mathrm{O}-18\right.$ or $\left.{ }^{18} \mathrm{O}\right)$. The deuterium washes out of the body as water while the ${ }^{18} \mathrm{O}$ washes out of the body as water and carbon dioxide. The difference between the two elimination rates is a measure of carbon dioxide output (Schoeller, 1988). Energy expenditure is then calculated from the carbon dioxide using standard equations. DLW may be administered by injection or orally. The loss of deuterium and ${ }^{18} \mathrm{O}$ in the subject is tracked by sampling the saliva, urine, or blood (Schoeller, Taylor, \& Shay, 1995). The reliability and validity of this instrument has long been established. This technique is very useful for measuring metabolic rates over a long period of time. It has been used extensively in animals and humans including infants and pregnant women. DLW is regarded as the gold standard for measuring energy expenditure under living conditions. This method is however expensive and requires technical expertise.

\section{Accelerometers}

Accelerometers are devices used to measure acceleration and the impact of gravity on the acceleration (Westerterp, 1999). Accelerometers are widely accepted as a physical activity measure because it offers an objective measurement and is equally inexpensive (Meijer, Westerterp, Verhoeven, Koper, \& ten Hoor, 1991). The accelerometer is not burdensome on the individual and is able to assess the intensity and 
duration of movement. There are two basic forms of accelerometers those that measure acceleration in three dimensions (triaxial) and those that measure acceleration in one dimension (uniaxial). Accelerometers are typically placed at the pelvic girdle and are capable of monitoring, storing and downloading data for relatively small time intervals over long period of time to capture the net forces acting on the whole body (Westerterp, 1999). Accelerometers have the capability to assess the intensity of physical activity as well as to detect vertical movement. Pedometers are unlikely to do both. Examples of uniaxial accelerometers include the Computer Science and Applications (CSA) and Actigraph while triaxial accelerometers include Triatrac-R3D, and Tracmor. The validity and reliability of some of these models are well established. Validation studies have yielded correlations ranging $\mathrm{r}=0.25-0.91$, for CSA $\mathrm{r}=0.89$, for Tracmor $\mathrm{r}=0.89$, for Actigraph $r=0.46-0.73$ (Wong, Webster, Montoye, \& Washburn, 1981). Although accelerators are well accepted as a technique for measuring energy expenditure, some fall backs have been reported such as inability to record static movement, lack of sensitivity to incline and to changes involving resistances.

\section{Heart Rate Monitor}

Heart rate monitor is a device that allows the user to measure their heart rate. An increase in heart rate is reflective of an increase in the intensity of physical activity. This can be affected by varying emotional states and environmental stimuli. The heart rate can also be monitored by checking the radial or carotid pulse using two fingers of one hand to locate the artery. Accurate pulse count is essential. Heart rate monitoring (not amount of perspiration produced) is usually the preferred way to monitor the intensity of physical activity given that there is a range that is safe and effective for promoting cardiovascular benefits (American Council on Exercise [ACE], 2001). To determine this range, the individual would have to estimate their maximal heart rate by subtracting their age from the number 220. This number will guide them to determine the number of beats per minute at which their heart should be beating during aerobic exercise. For most healthy individuals, this range is $50 \%$ to $80 \%$ of their maximal heart rate often referred to as the target heart rate zone. These numbers then serve as guidelines on the intensity of physical activity the individual should engage in.

\section{Physical Activity Questionnaires, Recalls, and Records}

Another common technique for assessing an individual's energy expenditure is physical activity record keeping in a diary. This is a self-report instrument that details patterns of physical activity performed by the individual. This method is cost effective; however, its greatest limitation is the demand it places on the individual and the burden it places on study staff that have to sort through large volumes of information. Recalls usually adopt an interview format and are useful to assess physical activity over a given period of time. The limitation of this technique lies in the ability of the individual to remember and report physical activity patterns with accuracy. Questionnaires are used to determine the frequency, intensity, duration, context, and type of activity performed by 
the participant. Although questionnaires may limit the individual's ability to give detailed information, their utility in physical activity assessment is well established. The 7-Day PAR Questionnaire is an example of a tool that utilizes this technique.

\section{Seven-Day Physical Activity Recall Questionnaire}

The Seven-Day Physical Activity Recall (7-Day PAR) Questionnaire assesses the individual's habitual physical activity pattern utilizing a one-week time frame (Blair et al., 1998; Sallis et al., 1985). This self-report instrument utilizes both the recall and questionnaire format to obtain information from the participant. The PAR has shown utility in exercise, epidemiological studies and a variety of scientific applications through the information it provides regarding activity volume, intensity, and duration.

To facilitate recall, the instrument is broken down into segments of morning, afternoon, and evening, and for each segment the participant is asked to report the amount in minutes of categories of moderate, hard, and very hard activity they performed. They can only report activities that took not less than 10 minutes to perform. The time spent sleeping is classified as "light activity." The PAR is scored by averaging the number of minutes spent in moderate, hard, and very hard activities. The time spent in each category is further multiplied by body weight and a caloric value to yield the total daily energy expenditure. The total energy expenditure is estimated by multiplying each category by an established metabolic equivalent, or MET value. For instance, sleep $=1$ MET, light activity $=1.5 \mathrm{MET}$, moderate activity $=4 \mathrm{METs}$, hard activity $=6 \mathrm{METs}$, and very hard activity $=10$ METs.

The validity and reliability of the 7-Day PAR instrument within the adult population has been long established. Johnson-Kozlow, Rock, Gilpin, Hollenbach, and Pierce (2007) found that the Women's Health initiative had comparable validity, sensitivity, and measurement bias compared to the widely accepted PAR (correlation = 0.73). Similarly, the validity of the Stanford 7-Day PAR in young adults was evaluated by Washburn, Jacobsen, Sonko, Hill, and Donnelly (2003). They noted no significant differences between the 7-Day PAR and the doubly-labeled water technique.

Daily physical activity has tremendous health benefits throughout a lifespan. Besides its impact in weight reduction, sustained physical activity enhances cardiovascular health in many ways, including improving endothelial function, improving lipid profile, and reducing the effects of obesity, diabetes, and hypertension. Physical activity benefits extend to both normal weight and obese persons irrespective of ethnicity. 


\section{Traditional Cardiovascular Disease Risk Factors}

\section{Historical Perspective}

Several years ago, high blood pressure was thought to be a normal part of the aging process and clinicians accepted the notion that atherosclerosis was inevitable. During those times, most clinicians were unaware of the association between hypercholesterolemia and heart attacks, and the concepts of risk stratification or modification of risk factors for cardiovascular disease were unknown. It was over half a century ago, when the Framingham Heart Study was conducted in Framingham, Massachusetts, that remarkable advances began in the prevention of cardiovascular disease not only in the United States but around the world. The Framingham Heart Study was a large scale public health service investigation examining why heart disease had become the leading cause of death in the 1940s. The quest to learn the biologic and environmental factors contributing to cardiovascular disability and death gave rise to the epidemiological study that was the Framingham Heart Study (Wilson et al., 1998).

\section{The Framingham Heart Study}

The first cohort of participants was 5,209 healthy male and female residents between 30 and 60 years of age from the town of Framingham. Later, in the early 1970s, children and their spouses from the original cohort group (5,124 persons) were recruited for a second study, the "Offspring Study" (Rutter, Meigs, Sullivan, D'Agostino, \& Wilson, 2004). Currently, about 500 members of the minority community at Framingham are being recruited for the "Omni Study" to see if these latest participants have similar or different risk factors associated with cardiovascular disease when compared with the other two cohorts, since participants in the original study were mostly white males.

Needless to say, the Framingham Heart Study was the landmark study that first identified the major risk factors for cardiovascular disease (Dawber, Meadors, \& Moore Jr., 1951). The study established the relationship between the levels of cholesterol and risk of disease. It further established a strong, positive association of LDL-cholesterol with cardiovascular disease as well as a powerful inverse and protective effect of HDLcholesterol levels.

Prior to the Framingham Heart Study, there was a widely held notion that women and the elderly tolerated higher blood pressures well. The study found no justification that the elderly coped better with hypertension than younger persons; neither did it find gender differences in cardiovascular disease risk in the presence of high blood pressure. The study further established the association between cholesterol levels, high blood pressure, diabetes, and heart disease. The Framingham Study also looked at lifestyle issues that contributed to the high rates of cardiovascular disease. The study researchers found that a lifestyle characterized by sedentary behavior, unhealthy diet, and uncontrolled weight gain exacerbated the risk factors leading to adverse cardiovascular conditions. The Framingham Heart Study identified high blood pressure, high blood 
cholesterol, smoking, obesity, diabetes, and physical inactivity as the major risk factors for cardiovascular disease (Wilson, D'Agostino, Sullivan, Parise, \& Kannel, 2002). The role of associated factors such as blood triglyceride, HDL-cholesterol, age, gender, and psychosocial issues was also highlighted.

The findings of the Framingham Heart Study have been validated in multiple clinical trials both in the United States and other countries around the world. The VERIFICA Study of Spain assessed the reliability and accuracy of the Framingham coronary heart disease risk prediction model and determined that it reliably predicted the 5 -year coronary heart disease risk for patients aged 35 to 74 years in their population (Marrugat et al., 2007). Likewise, Japanese investigators used the Framingham risk model to accurately evaluate more than 5,000 male subjects and confirmed that it provided useful information on future coronary heart disease events in Japanese men (Suka, Sugimori, \& Yoshida, 2002). Other investigators, such as D'Agostino, Grundy, Sullivan, and Wilson (2001), evaluated the applicability of the Framingham risk model to multiple ethnic groups in six different prospective studies and confirmed that it was applicable to other ethnic groups after recalibration for differences in the prevalence of risk factors and underlying rates of coronary heart disease events.

The Framingham Heart Study has been helpful in identifying traditional cardiovascular risk factors; studies such as the Jackson Heart Study (JHS) have confirmed its utility in African Americans also (Taylor et al., 2008). The JHS found that $40 \%$ of African American women, as opposed to $29 \%$ of African American men, had the traditional cardiovascular risk factors (Association of American Medical Colleges [AAMC], 2008). The JHS study was an outgrowth of the Atherosclerosis Risk in Communities (ARIC) study, which is an ongoing all-African American study of men and women ages 35 to 84 that seeks to investigate the causes of cardiovascular disease in African Americans in order to learn how to best prevent this group of diseases in the future. To the best of our knowledge, the findings of this study are yet to be published, though information about the study's methodology is discussed in available literature. Our study's strength lies in its population, age, and the novelty of our topic of investigation.

African American women continue to bear a disproportionate burden of cardiovascular disease mortality and morbidity despite increased awareness of cardiovascular risk and growing intervention efforts. While hypertension is more prevalent among African Americans, at similar levels of adiposity, African Americans have a more protective lipid profile than Caucasians (Kullo, Jan, Bailey, Mosley, \& Turner, 2007). It has become obvious that the traditional cardiovascular disease risk factors (hypertension, dyslipidemia, diabetes, smoking, and physical inactivity) are inadequate to explain the increased incidence of cardiovascular disease in African American women. 


\section{Emerging Cardiovascular Disease Risk Factors}

\section{Overview}

Although the Framingham coronary risk score has facilitated the identification of individuals at high risk for cardiovascular disease, researchers now argue that the classical risk factors used in these algorithms do not adequately differentiate individuals at moderate risk (Ferns, 2008). The death toll from atherosclerotic cardiovascular disease continues to rise (WHO, 2004) despite major advances in treatment of ischemic heart disease. Unfortunately, a large number of victims of the disease who are apparently healthy die suddenly without prior symptoms (Kanjilal et al., 2008). Available screening and diagnostic methods are insufficient to identify the victims before the event occurs. Current knowledge portend that atherosclerosis is not only a lipid disorder but an inflammatory one as well (Libby, Ridker, \& Maseri, 2002). The additional use of inflammatory biomarkers such as high sensitivity C-reactive protein (hs-CRP), Plasminogen Activator Inhibitor-1 (PAI-1), soluble Intercellular Adhesion Molecule-1 (ICAM-1), E-selectin, QUICKI, and fibrinogen can improve methods of global cardiovascular risk assessment (Albert \& Ridker, 2006).

\section{Emerging CVD Risk Markers}

Several investigators have examined the utility of these emerging markers in cardiovascular disease stratification. Mora, Rifai, Buring, \& Ridker (2006) studied associations of baseline immunoassay fibrinogen and hs-CRP measurements with incident cardiovascular disease. This prospective study of almost 28,000 initially healthy women who were followed for 10 years found that baseline fibrinogen level provides additive value to hs-CRP and traditional risk factors in predicting incident cardiovascular disease. Similarly, Smith et al. (2005) followed over 2,000 men for a median of 13 years to see whether hemostatic markers contribute to risk of coronary disease and ischemic stroke independently of conventional risk factors. Univariate analysis revealed that fibrinogen, hs-CRP, D-dimer, tissue plasminogen activator (tPA) and plasminogen activator inhibitor-1(PAI-1) were associated significantly with risk of cardiovascular disease while factor VIIc showed an inverse association $(\mathrm{p}=0.001)$.

Another study examined cross-sectional relationships between the concentrations of soluble adhesion molecules (sICAM-1, sVCAM-1, sP-selectin, and sE-selectin) and smoking behavior, body composition, blood pressure, serum lipids, and physical activity in a large sample of about 600 healthy men and women, with special emphasis on interactions between smoking and other cardiovascular disease risk factors (Demerath, Towne, Blangero, \& Siervogel, 2001). The findings indicated no gender differences in the concentrations of sICAM-1, sVCAM-1, and sP-selectin, but men had higher sEselectin levels than women $(\mathrm{p}<0.0001)$. Male and female smokers had higher sICAM- 1 and sE-selectin levels than non-smokers, and soluble cell adhesion molecules (CAMs) were correlated with pack-years of cigarette smoking $(r=0.3-0.4, p<0.0001$, 
significant in women only). Significant independent associations were found between soluble CAMs and smoking, waist-hip-ratio (WHR), blood pressure, HDL-cholesterol, and total cholesterol. This result showed that concentration of soluble CAMs, particularly sICAM-1 and sE-selectin, reflect the level of established cardiovascular disease risk factors in apparently healthy men and women, thus adding to the evidence that these factors contribute to cardiovascular disease through their inflammatory effects on the vascular endothelium.

Ridker, Hennekens, Buring, \& Rifai (2000) conducted a prospective case-control study among over 28,000 apparently healthy postmenopausal women. These subjects were followed for about 3 years to assess the risk of cardiovascular events associated with base-line levels of hs-CRP, serum amyloid A, interleukin-6, and soluble intercellular adhesion molecule-1 (sICAM-1), all markers of inflammation. The results indicated that hs-CRP was the strongest univariate predictor of the risk of cardiovascular events. Other markers significantly associated with the risk of cardiovascular events were serum amyloid A, sICAM-1, and interleukin-6. They also noted that prediction models that incorporated markers of inflammation in addition to lipids were significantly better at predicting risk than models based on lipid levels alone $(\mathrm{p}<0.001)$.

Thus, these emerging risk factors may play a role in the racial/ethnic differences in cardiovascular disease rates. Elevated concentrations of hemostatic variables such as fibrinogen and PAI-1 have been found in both African American and Caucasian overweight/obese women (Perry et al., 2007). African American women have some of the highest levels of fibrinogen and E-selectin but among the lowest levels of intercellular adhesion molecule-1 and PAI-1, while Caucasians and Hispanics tended to have intermediate levels of these factors and markers. Similarly, insulin resistance has been associated with increased cardiovascular risk, including dyslipidemia, hypertension, and impaired fibrinolysis - particularly in nondiabetic persons. Additionally, a multiethnic study of adults (Howard et al., 1998) found greater insulin resistance was associated with lipid abnormalities, including elevated LDL and low HDL cholesterol, as well as LDL size and higher triglycerides. It is recognized that obesity is associated with higher insulin levels and greater insulin resistance regardless of race (Gower, 1999). However, racial differences in hyperinsulinemia and insulin resistance are present independent of adiposity (Velasquez-Mieyer et al., 2000). Lean and obese African Americans exhibiting higher fasting and stimulated insulin levels than Caucasians (Dowling, Fried, \& PiSunyer, 1995; Haffner et al., 1996; Jiang, Srinivasan, Radhakrishnamurthy, Dalferes, \& Berenson, 1996) and greater insulin resistance (Arslanian, Suprasongsin, \& Janosky, 1997). These higher insulin levels and greater insulin resistance may be underlying mechanisms for the higher morbidity and mortality seen in African Americans.

Literature is still sparse regarding the role of inflammation in concert with obesity in predicting cardiovascular risk in African American women. The POWIRS (Profiles of Obese Women with the Insulin Resistance Syndrome) Study of South Africa examined the relationship between inflammation, obesity, and cardiovascular disease in apparently healthy African and Caucasian women (Schutte et al., 2005). The results showed that the levels of leptin, hs-CRP, and fibrinogen were significantly higher $(p<0.05)$ in African American women. However, except for leptin levels, no other cardiovascular parameters 
explained the variation in the inflammatory markers. There is a growing need to examine the role of these emerging markers in African American women.

\section{Summary}

The United States and many other countries are experiencing an epidemic of obesity. Physical inactivity, which is more prevalent in women and ethnic minority population's particularly African American women, and poor nutrition have been some of the reasons behind the weight gain. Epidemiological studies have documented a strong associated between obesity and traditional cardiovascular disease risk factors (Freedman, Khan, Serdula, Galuska, \& Dietz, 2002; Nasir et al., 2007). In recent years, literature has begun to accumulate regarding the role of inflammation in atherosclerosis formerly thought to be a lipid disease. Compelling evidence for the importance of inflammation in atherosclerosis has continued to evolve. Cross-sectional studies have reported associations between obesity and different inflammatory markers (Festa et al., 2001; Ford, 1999; Yudkin, Stehouwer, Emeis, \& Coppack, 1999). It is possible that the relation between inflammation and incidence of cardiovascular diseases is mediated through an increased incidence of the traditional risk factors. However, there is a paucity of data regarding the impact of obesity on traditional and emerging risk factors for cardiovascular disease in African American women. This pilot study will provide insight into the relationship between obesity and cardiovascular disease risk factors in this at-risk group of women. 


\section{CHAPTER 3. METHODOLOGY}

There is an increasing body of evidence that the traditional cardiovascular disease risk factors alone are inadequate to explain the high rate of cardiovascular disease morbidity and mortality among African American women (Libby et al., 2002; Ridker, Hennekens, Buring, \& Rifai, 2000). Current research suggests that inflammatory, thrombotic, and endothelial dysfunction markers such as high sensitivity C-reactive protein (hs-CRP), plasminogen activator inhibitor-1 (PAI-1), fibrinogen, soluble intercellular adhesion molecule-1 (sICAM-1), and E-selectin may provide additional prognostic information about risk of future coronary events in apparently healthy subjects (Ridker, 1999; Ross, 1999) . This chapter presents a description of the research design, sample and setting, instruments, procedures, statistical analyses, and human subject considerations used in this study.

\section{Research Design}

This study utilized a cross-sectional, descriptive, correlational design to examine the impact of obesity on the traditional and emerging cardiovascular disease risk factors in adult African American women. A cross-sectional design was employed in the current study because it provides information about lifestyle practices and to examine the relationships among study variables, such as physical activity levels, illicit drug use, smoking habits, age, education, marital status, and income levels of a select group of African American women. The correlational design was used to describe the direction and magnitude of the relationship between body weight and cardiovascular disease risk factors. Descriptive statistics were used to describe the basic features of the data in this study. Burns (1993) noted that correlational studies examine relationships between variables; thus, statistical correlational methods were used to examine the relationships that existed between the independent variables (obesity and normal weight) and dependent variables (traditional and emerging cardiovascular disease risk factors) in African American women. Traditional cardiovascular disease risk factors examined included hypertension, diabetes mellitus, family history of cardiovascular disease, tobacco use, physical inactivity, and dyslipidemia. Fasting blood draws were performed to check levels of the following emerging risk factors: QUICKI, hs-CRP, PAI-1, fibrinogen, sICAM-1, and E-selectin.

\section{Sample and Setting}

A non-probability, convenience sample of 50 African American women who met the inclusion criteria were recruited for participation in the study. Since this was an

exploratory study that does not usually lend itself to a power analysis, a sample size of 50 was deemed adequate. Two of the subjects did not complete all study procedures and were excluded from the analysis. Recruitment of subjects for the study was conducted from three urban geographic locations in the southern region of the United States. The 
subjects were recruited from mid-south family practice/internal medicine clinics and Health Works, a program designed to transition women off welfare into the workforce. The data collection sites included Mississippi, middle Tennessee, and west Tennessee. The study participants were healthy African American women who self-reported that they had no known cardiovascular disease. Participants were classified as obese or normal weight based on their BMI (Figure 3.1).

\section{Inclusion Criteria}

The following inclusion and exclusion criteria were established to identify subjects for this investigation.

- Self-identify as African American woman

- 18 to 45 years of age at the time of enrollment

- Body mass index (BMI) of 18.5 to $24.9 \mathrm{~kg} / \mathrm{m}^{2}$ for the normal weight category or a $\mathrm{BMI} \geq 30 \mathrm{~kg} / \mathrm{m}^{2}$ for the obese category

- Able to read and write in English language

- Sign informed consent form prior to participation

\section{Exclusion Criteria}

- Pregnancy

- Use of hormone replacement therapy, steroids, and/or cholesterol lowering medications such as statins

- Personal history of gastric bypass surgery

- Personal history of cardiovascular disease including coronary artery disease (CAD), myocardial infarction (MI), congestive heart failure (CHF), and cerebrovascular accident (CVA)

- Inability or unwillingness to give written informed consent

Study Population

Figure 3.1 Study Sample 


\section{Instruments}

Measurements of traditional and emerging risk factors were obtained at the same study visit. Additionally, subjects completed a demographic questionnaire which was used to characterize the sample.

\section{Urine Pregnancy Test}

A urine pregnancy test was performed to determine eligibility for the study. Individuals with a positive pregnancy test were not eligible to participate in the study; hence no further study measures were performed. The urine pregnancy test determines the presence of human chorionic gonadotropin (hCG) hormone in early pregnancy urines (Wilcox, Baird, Dunson, McChesney, \& Weinberg, 2001). The Natureplex Be Certain Home Pregnancy Test (Memphis, TN), an over-the-counter home pregnancy testing device that claims $>99 \%$ diagnostic accuracy for pregnancy and utility on the first day of the missed menses or earlier, was used for the study. Participants were instructed to collect their urine samples in a clean labeled container for easy identification. Three drops of urine sample were put into the sample well and the unit was laid flat and read within 5 minutes. The presence of only one pink color control band within the test means that the individual was not pregnant, while the presence of two pink control and test bands within the test means that the individual was likely to be pregnant and should consult her primary care provider. The significance of eliminating pregnant women from the study lies in the fact that recent studies have documented associations among pregnancy, obesity, and cardiovascular disease risk markers. Pregnancy has been noted to increase the levels of the inflammatory markers such as C-reactive protein and fibrinogen (Ridker, Hennekens, Buring, \& Rifai, 2000). Similarly, serum levels of total cholesterol, HDL-cholesterol, LDL-cholesterol and triglycerides have been noted to increase substantially by the third trimester (Glew et al., 2004).

\section{Demographic Questionnaire}

The participants completed an investigator-developed questionnaire on health history and issues related to cardiovascular disease (Appendix A). This instrument was written at a 6th grade reading level and had a multiple-choice response format. Various demographic variables were assessed, including age in years, marital status, education level classified by degree earned, income level, history of chronic diseases, medication and social history, illicit drug use, and history of gastric bypass. All were classified by yes/no based on self-report. The demographic variables were analyzed using descriptive statistics. Additionally, tobacco use and family history of cardiovascular disease, two of the traditional cardiovascular risk factors, were ascertained through self-report on the demographic questionnaire. 


\section{Seven-Day Physical Activity Recall Questionnaire}

The Seven-Day Physical Activity Recall (7-Day PAR) Questionnaire was developed over two decades ago as a general-purpose measure of physical activity (Sallis et al., 1985; Appendix B). This self-report recall instrument was used in this study to assess respondent's physical activity level and can be completed in about 20 minutes. This instrument provides detail regarding the intensity, time (duration), and type of physical activity in a one-week time frame.

The 7-Day PAR instrument breaks down the day into three segments: morning, afternoon, and evening to facilitate recall. Research subjects were asked to report the number of hours they spent sleeping and the remaining time is grouped as "light" activities. They were also asked to report the amount of moderate, hard, and very hard activity that they performed in the last seven days. The different activity levels were assigned Metabolic Equivalent (MET) values. Time spent in sleep (1 MET), "light" activities (1.5 METs), moderate (3 METs), hard (6 METs), and very hard (9 METs) activities for the past 7 days were multiplied by their respective MET values. The estimate of the total kilocalories (kcal) of energy expenditure per day is calculated by multiplying the total METs by the individual weight in kilograms and divided by seven (number of days) and the result was expressed as $\mathrm{kcal} / \mathrm{kg} / \mathrm{d}$. Additionally, the number of days per week individuals self-reported engaging in 30 minutes or more of at least moderate physical activity was assessed. The validity and reliability of the 7-Day PAR instrument within multi-ethnic adult population has been long established. JohnsonKozlow, Rock, Gilpin, Hollebenk, and Pierce (2007) found that the Women's Health Initiative had comparable validity, sensitivity and measurement bias compared to the widely accepted PAR $(r=0.73)$. Similarly, the validity of the Stanford 7-Day PAR in young adults was evaluated by Washburn, Jacobsen, Sonko, Hill, and Donnelly (2003). They noted no significant differences between the 7-Day PAR and the doubly-labeled water group. Ainsworth et al. (2000) analyzed the physical activity patterns of 141 African American and Native American women, ages 40 and older, enrolled in the CrossCultural Activity Participation Study. Using the 7-Day PAR instrument, they found that physical activity participation patterns differed by ethnicity, with relatively more Native American women reporting moderate intensity walking for exercise, occupational activities and child care activities compared with African American women $(p<0.05)$.

\section{Blood Pressure and Heart Rate Screening}

The study participants' blood pressure and heart rate were measured following the guidelines provided by the Seventh Joint National Committee (JNC 7, 2003) on the Prevention, Detection, Evaluation, and Treatment of High Blood Pressure. The principal investigator used the auscultatory method of blood pressure measurement with a random zero sphygmomanometer to obtain blood pressure. Participants were required to sit comfortably for five minutes with the back and arm supported, the legs uncrossed, and the upper arm at the level of the right atrium before the first reading was taken. Two readings were taken five minutes apart with cuff on the right arm and the study participant seated. An appropriate-sized cuff with the cuff bladder able to encircle at least 
$80 \%$ of the arm was used to ensure accuracy of the results. Two blood pressure measurements were averaged and the average blood pressure was classified using the protocol. Systolic blood pressure 120 to $139 \mathrm{mmHg}$ or diastolic blood pressure of 80 to $89 \mathrm{mmHg}$ was classified as prehypertension. Participants' with a systolic blood pressure of 140 to $159 \mathrm{mmHg}$ or a diastolic blood pressure of 90 to $99 \mathrm{mmHg}$ were described as Stage 1 hypertension. Similarly, participants with systolic blood pressure of greater or equal to $160 \mathrm{mmHg}$ or diastolic blood pressure of greater or equal to $100 \mathrm{mmHg}$ were classified as Stage 2 hypertension. Systolic blood pressure that is less than $120 \mathrm{mmHg}$ or diastolic blood pressure less than $80 \mathrm{mmHg}$ was described as normal blood pressure.

\section{Anthropometric Assessment}

Height, weight, waist, and hip measurements were obtained from each participant by the same investigator. Subjects were required to remove shoes and heavy outer garments before the measurements of height and weight. Height was obtained using a scale-mounted measuring tool and measured to the nearest centimeter. A calibrated balance scale or an electronic scale was used to assess subject's weight to the nearest tenth of a kilogram. Although different weight scales were used at each of the different locations, they were standardized to no more than a 0.05 pound difference by weighing the principal investigator on each of the scales. The subjects' height and weight were measured following the World Health Organization's MONICA [Monitoring Trends and determinants in Cardiovascular disease] project guidelines (Molarius, Seidell, Sans, Tuomilehto, \& Kuulasmaa, 2000). Body mass index (BMI) was calculated using each individual weight in kilograms divided by their height in meters squared (WHO, 2000). Waist and hip circumference were measured to further characterize the sample. The same tape measure was used for all the subjects to measure the waist and hip. The waist was measured midway between the bottom of the ribs and the top of the hip bone and hips at the widest point between the hips and buttocks in the standing position and the waist-hipratio (WHR) was calculated in centimeters. WHR was computed as waist divided by hip (WHO, 2000). Similarly, WC was measured around the bare abdomen using the same measuring tape at the nearest $0.1 \mathrm{~cm}$ at the high point of the iliac crest at minimal respiration (CDC, 2005). The NCEP ATP III criteria for defining abdominal obesity as a WC $>35$ inches $(88 \mathrm{~cm})$ in women and $>40$ inches $(102 \mathrm{~cm})$ in men (Expert Panel on Detection, Evaluation, and Treatment of High Blood Cholesterol in Adults, 2001) was used as well as the WHO parameter for abdominal obesity which is WHR of $>0.80$ in women or $>0.90$ in men (Medscape, Connect, Care, Surgery, \& Today, 2005; Pouliot et al., 1994).

\section{Blood Collection for Traditional and Emerging Risk Factors}

After a 10-hour overnight fast, blood samples for glucose, insulin, hs-CRP, fibrinogen, PAI-1, sICAM-1, E-selectin, total cholesterol, LDL-cholesterol, HDLcholesterol, and triglycerides, were obtained. The procedure for blood sample collection was discussed with each participant. There was no associated cost regarding the blood 
collection to the participant. The principal investigator carried all needed supplies to each of the recruitment sites: the Center for Health Evaluation and Lifestyle Promotion at the University of Tennessee Health Science Center, Internal Medicine and Family Practice clinics as well as collected the blood samples. Possible risk of bruising at the venipuncture site was shared with participants.

About $30 \mathrm{cc}$ of blood was collected from each participant into BD vacutainer SST tube for serum determinations in chemistry and lavender tubes containing EDTA for anticoagulation. Each tube was inverted after collection to prevent clotting. Samples were stored in ice for not more than 2 hours until they were centrifuged. The plasma and serum was separated into cryo tubes and stored at $-80{ }^{\circ} \mathrm{C}$ until processing. Instructions regarding proper handling of serum/plasma samples as well as overnight mailing and shipping procedures were adhered to. Sample analyses were conducted at the University of Miami and the University of Tennessee Health Science Center using the procedures described in the following sections.

\section{Traditional Cardiovascular Risk Factors}

\section{Glucose}

Plasma glucose was measured in triplicate using the glucose oxidase method (Kadish \& Hall, 1965). The American Diabetes Association (2007) diagnostic criteria were used to identify impairment in glucose metabolism. Fasting plasma glucose levels of $100-125 \mathrm{mg} / \mathrm{dl}$ were classified as impaired fasting glucose (IFG), while levels $\geq 126$ $\mathrm{mg} / \mathrm{dl}$ were classified as diabetes mellitus.

\section{Lipid Analyses}

The chemistry assays were performed by the University of Miami Diabetes Research Institute on the automated analyzer (Roche Cobas-Mira) utilizing the manufacturer's commercially available kits. Instrument set-up, run procedures, and maintenance policies were strictly adhered to according to the manufacturer's instructions. The description of the laboratory procedures was provided by Dr. Armando Mendez, co-director of the laboratory (personal communication, August 22, 2008). Normal values for total cholesterol $(<200 \mathrm{mg} / \mathrm{dl})$, LDL-cholesterol $(<130 \mathrm{mg} / \mathrm{dl})$, HDLcholesterol $(\geq 50 \mathrm{mg} / \mathrm{dl})$, and triglyceride levels $(<150 \mathrm{mg} / \mathrm{dl})$ are based upon the National Cholesterol Education Program criteria (Cleeman \& Lenfant, 1998; Executive Summary of The Third Report of The National Cholesterol Education Program (NCEP) Expert Panel on Detection, Evaluation, and Treatment of High Blood Cholesterol in Adults [Adult Treatment Panel III], 2001). 


\section{Total Cholesterol}

Plasma cholesterol was measured after release from its esters by an ester hydrolase. The free cholesterol was then oxidized by cholesterol oxidase, producing hydrogen peroxide. The hydrogen peroxide, when combined with 4-aminoantipyrine and phenol, forms a chromophore in an amount which is directly proportional to the cholesterol concentration in the sample and is quantitated photometrically at $540 \mathrm{~nm}$ (Allain, Poon, Chan, Richmond, \& Fu, 1974). Intra-assay and inter-assay CVs are 2.5 and $3.5 \%$, respectively (A. Mendez, personal communication, August 22, 2008).

\section{HDL-Cholesterol}

Dextran sulfate $(50,000 \mathrm{MW})$ and magnesium precipitate low density lipoproteins (LDL) and very low density lipoproteins (VLDL) in plasma or serum. After centrifugation, the HDL remains in the supernatant. The inter-assay CV is $2.5 \%$ (A. Mendez, personal communication, August 22, 2008).

\section{LDL-Cholesterol}

LDL-cholesterol was calculated using the Friedewald formula (Friedewald, Levy, $\&$ Fredrickson, 1972), which states that LDL-cholesterol = total cholesterol - (fasting triglyceride/5) - (HDL-cholesterol). This method has been shown to correlate well with ultracentrifugally derived LDL-cholesterol values up to triglyceride values of $400 \mathrm{mg} / \mathrm{dl}$.

\section{Triglyceride}

Plasma triglyceride level was measured after hydrolysis by lipoprotein lipase to glycerol and fatty acids. Glycerol was enzymatically phosphorylated and then oxidized to release hydrogen peroxide that peroxidized to form a quinoneimine chromophore which can be read at $490 \mathrm{~nm}$ in the spectrophotometer. The inter-assay $\mathrm{CV}$ is $5.3 \%$. (A. Mendez, personal communication, August 22, 2008).

\section{Emerging Cardiovascular Risk Factors Screening}

These tests were performed on the Dade-Behring nephelometer using the manufacturer's reagents, quality controls, and methods. The nephelometer measures the light scatter due to the formation of immune complexes between standards or unknowns and specific antibodies. The extent of light scatter was directly proportional to the antigen concentration. All tests utilize a serum standard that was diluted to generate a standard curve and to minimize matrix effects in this matrix-sensitive methodology. Instrument set-up, run procedures, and maintenance policies were strictly adhered to according to the manufacturer's instructions. Reagents used in the nephelometer were obtained from Dade-Behring. 


\section{Fibrinogen}

Diluted EDTA plasma was incubated with rabbit anti-human fibrinogen antibody to form immune complexes. The serum standard has a reference value in the range 2.0 $2.5 \mathrm{~g} / \mathrm{l}$. The normal range is from $1.8-3.5 \mathrm{~g} / 1$ and the intra-assay and inter-assay CVs are $2.7 \%$ and $2.6 \%$, respectively (Ernst, 1993). Normal laboratory values are estimated at $200-350 \mathrm{mg} / \mathrm{dl}$ (A. Mendez, personal communication, August 22, 2008).

\section{High Sensitivity C-reactive Protein (hs-CRP)}

Diluted serum or EDTA plasma is incubated with polystyrene particles coated with monoclonal antibodies to CRP to produce agglutination and light scattering in proportion to the concentration of antigen. The serum standard was diluted to produce a standard curve from 0.08 to $6 \mathrm{mg} / \mathrm{l}$, and healthy individuals are expected to have values of $<3 \mathrm{mg} / \mathrm{l}$. Mean values for men with and without myocardial infarction were 1.51 and $1.13 \mathrm{mg} / \mathrm{l}$, respectively (Rifai, Tracy, \& Ridker, 1999). Intra-assay and inter-assay CVs are $<4.4 \%$ and $<5.7 \%$, respectively. Hs-CRP values range between 0.3 and $8.6 \mathrm{mg} / \mathrm{L}$ in healthy men and between 0.2 and $9.1 \mathrm{mg} / \mathrm{L}$ in healthy women (Ridker, 2008). The following interpretation of hs-CRP results has been recommended by the Center for Disease Control and Prevention (CDC) and the American Heart Association (AHA) for cardiovascular disease risk classification: $<1 \mathrm{mg} / \mathrm{L}=$ low risk, $1-3 \mathrm{mg} / \mathrm{L}=$ average risk and $>3 \mathrm{mg} / \mathrm{L}=$ high risk (A. Mendez, personal communication, August 22, 2008).

\section{Plasminogen Activator Inhibitor-1 (PAI-1), E-selectin, and Soluble ICAM-1}

The simultaneous quantification of the following analytes-Plasminogen Activator Inhibitor-1 (PAI-1, total), E-selectin, and sICAM-1-were performed using the multiplex assay kit manufactured by Millipore Corporation (St. Charles, MO). Prior to beginning the assay, a diagram placement of background, standards, controls, and serum samples on Well Map Worksheet was set-up in a vertical configuration and the assay was run in duplicate. Consistent with manufacturer's guidelines, appropriate matrix solution was added to the Background, Standards, and Control wells. Assay buffer was used as matrix solution before adding the samples into the appropriate wells. Mixed beads were subsequently added, sealed, and covered with aluminum foil and placed overnight on a plate shaker. Vacuum filtration was used to remove the wash buffer before adding Streptavidin-Phycoerythrin, which was placed in a plate shaker for 30 minutes. Vacuum filtration was also done afterwards to wash buffer twice before the plate was finally run on a Luminex instrument. The following values are used in clinical research for healthy controls: Soluble ICAM-1 range is $98.8-320 \mathrm{ng} / \mathrm{ml}$, E-selectin range is $17.9-79.2 \mathrm{ng} / \mathrm{ml}$, while PAI-1 is $21.0+/-7.2 \mathrm{ng} / \mathrm{ml}$ (LINCO Research, 2005). 


\section{Insulin}

Serum fasting insulin was measured in serum samples using a solid phase 125I radioimmunoassay (Coat-A-Count, Diagnostic Products Corp.) at the University of Miami Diabetes Research Institute. The assay utilizes a tube-immobilized anti-human insulin antibody that provides a convenient overnight (or, alternatively, a 3-hour) incubation after addition of cold antigen and 1251-insulin label in buffer, followed by simple decanting of the supernatant, and gamma counting. The assay is sensitive down to $1.2 \mu \mathrm{U} / \mathrm{ml}$. At mid-curve there is $40 \%$ cross-reactivity for proinsulin. Intra-assay and interassay CVs are $3.1-9.3 \%$ and $4.9-10.0 \%$, depending on antigen concentration (A. Mendez, personal communication, August 22, 2008). Quantitative insulin sensitivity check index [QUICKI] (Katz et al. 2000) was derived using the formula $1 / \log$ insulin + $\log$ glycemia in $\mathrm{mg} / \mathrm{dl}$. QUICKI values $<0.3$ indicate insulin resistance (VelasquezMieyer, Cowan, Neira, \& Tylavsky, 2008).

\section{Data Collection Procedure}

Permission to conduct this study was obtained from the University of Tennessee Institutional Review Board (IRB); see Appendix C. Recruitment was conducted through IRB-approved flyers (Appendix D) and contact with area nurse practitioners as well as Family Practice and Internal Medicine clinic owners across two southern states. Verbal approval of the Director of Health Works was obtained to recruit participants from that facility for the study.

\section{Site Preparation}

IRB-approved recruitment posters and fliers were placed in visible areas within the college community and area family/internal medicine clinics about three weeks prior to the actual date for data collection. The contact telephone number of the principal investigator was included on the fliers to answer questions and schedule participants for data collection. Volunteer participants were screened for eligibility to participate in the study, and instructions on the study protocol were provided. The information packet was given to participants in addition to IRB-approved Consent Form (Appendix E), demographic questionnaire, 7-Day PAR Questionnaire and anthropometric assessments. Participants were required to have an overnight fast of 10-12 hours prior to blood collection for analysis. A heart healthy diet was provided for participants following completion of the study protocol.

\section{Selection of Participants}

Individuals who were interested in the study indicated their willingness and the principal investigator provided them with an overview of the study aims, procedures, risks, and benefits. A date for testing was set for eligible individuals and instructions 
were given to fast for 10 hours prior to the testing session. On the day of testing, the study protocol was explained, questions were answered, and a written informed consent was obtained from qualified participants.

\section{Data Collection}

Study criteria checklist was presented to all participants to establish eligibility for the study. Verbal permission to perform urine pregnancy test was received. Participants were instructed to provide voided specimen for testing. Height and weight were measured to calculate BMI. Eligible participants continued with the remaining requirements for the study. The remaining testing procedures were initiated in the following sequence: resting blood pressure; completion of demographic and 7-Day PAR Questionnaires; waist, hip measures, and finally blood collection. About $30 \mathrm{cc}$ of venous blood was collected by the investigators. The questionnaires contained no personal identifying information; only codes were used for purposes of data analysis.

The completed questionnaires were reviewed by the principal investigator at the time of collection. Study questionnaires with missing data were returned to the participant to review and complete. The principal investigator provided assistance to participants with missing data, if needed.

Participants who completed all study protocol received a $\$ 20.00$ cash gift as an appreciation for their participation. Study participants signed a form acknowledging receipt of the cash gift. The form was co-signed by the investigators and stored in a secure location.

\section{Data Transfer}

The data collected from the questionnaires, anthropometric measures, and laboratory results were recorded in a password-protected Microsoft Excel computer spreadsheet. All data were coded to protect participants' confidentiality and promote anonymity. Laboratory results were shared with participants as they became available, along with a recommendation to review study results with their primary care providers.

\section{Data Analysis}

Data analysis was conducted using SAS version 9.1.3 (Cary, NC). Statistical analysis for this study included descriptive statistics. The study sample was characterized using measures of central tendency (means and median), variability (range and standard deviation), and frequency distributions (symmetrical and asymmetrical). Descriptive data consisting of age, income level, tobacco use, illicit drug use, BMI, WHR, WC, blood pressure, physical activity levels, blood glucose, insulin, QUICKI, lipid profile, hs-CRP, fibrinogen, PAI-1, sICAM-1, and E-selectin levels were used to summarize the 
demographic data and cardiovascular risk factors for the entire sample based on the total sample and by their weight categories. The relationships and degree of association among the variables were examined using Spearman's Rho correlation coefficients. Differences in risk factors between the obese and normal weight groups were explored using the Mann Whitney Wilcoxon test for continuous variables and chi-square for nominal variables. For inferential statistics, an alpha level of 0.05 was set a priori for statistical significance.

\section{Specific Aim One}

Describe the prevalence of traditional and emerging cardiovascular risk factors in African American women, ages 18 to 45, without a known history of cardiovascular disease.

The prevalence of traditional and emerging cardiovascular risk factors was determined by the proportion of the sample that had the risk factor divided by the total number of individuals in the sample. Descriptive statistics were used to derive information regarding the data distribution, mean, median, range, standard deviation, and frequency distributions of the study sample.

\section{Specific Aim Two}

Examine the relationship between BMI, physical activity levels, and other traditional (diabetes, dyslipidemia, tobacco use, family history of CVD, hypertension) and emerging (QUICKI, hs-CRP, PAI-1, fibrinogen, E-selectin, sICAM-1) cardiovascular disease risk factors in African American women.

Correlation analyses were computed to determine the interrelationship among the study variables (age, BMI, physical activity levels [kcal/week, hours of moderate weekly activity, days/week of moderate activity], weight, height, waist circumference, waist-hipratio, and traditional and emerging cardiovascular risk factors). Spearman Rho correlation coefficient ( $r$ ) was used to approximate the magnitude and direction of relationships among the variables. Graphic plots were performed to facilitate the visualization of the relationship between the variables of interest and to determine their linearity.

\section{Specific Aim Three}

Compare traditional and emerging risk factors among normal weight and obese African American women.

Since non-parametric statistics are most appropriately used when the sample sizes are small, the Mann Whitney $U$ test was used to examine the differences in cardiovascular risk factors between the normal weight and obese groups. For traditional 
risk factors and some emerging risk factors, normative values were used to determine whether an individual was classified as having the risk factor. The chi-square test provided an assessment of differences in the frequency of the risk factors between the normal weight and obese groups.

\section{Consideration of Human Subjects}

The study received approval from the University of Tennessee Health Science Center Institutional Review Board. Appropriate guidelines were maintained to ensure anonymity and confidentiality of subject participants. A unique identification code was assigned to each subject to facilitate data analysis and data collection forms were stored in a secure separate location. Blood samples were collected and stored in tubes labeled with the unique identification code in a $-80{ }^{\circ} \mathrm{C}$ freezer for batch analysis. Samples were sent to the laboratory without personal identification and data were reported on an Excel spreadsheet using the identification codes. 


\section{CHAPTER 4. RESULTS}

\section{Introduction}

The purpose of this study was to examine the impact of body weight on the traditional and emerging risk factors for cardiovascular disease in young African American women who have no history of cardiovascular disease. A general description of the sample population and their cardiovascular risks is provided in the following paragraphs. Additionally, the relationship between cardiovascular risk factors was explored. The results and statistical analyses of each research question are presented in this chapter.

\section{Description of the Sample}

The study sample consisted of 48 African American women from the mid-south. The mean age of the women was 31.58 (range 18-45 years). The sample description is provided in Table 4.1 for the whole group. The majority of the women $(62.5 \%)$ were unmarried, with about $35.4 \%$ having completed or almost completed high school/GED, while $64.6 \%$ had some form of college education. Similarly, $62.5 \%$ of the women were employed, while $37.5 \%$ were unemployed. Three-fourths $(75 \%)$ of the women reported an annual household income $<\$ 50,000$, while $14.58 \%$ of the participants had an annual household income $>\$ 100,000$. Five $(10.42 \%)$ of the women in our study admitted to the use of marijuana, while the remainder of the participants denied any use of illicit drugs. The vast majority of our participants denied any preexisting medical condition; only 2 $(4.17 \%)$ of the women reported a medical history of hypertension and diabetes.

\section{Research Findings}

Study findings are reported for each specific aim and question in sequence, with each aim restated, followed by the results of the statistical analyses.

\section{Primary Aim One}

Describe the prevalence of traditional and emerging cardiovascular risk factors in African American women, ages 18 to 45, without a known history of cardiovascular disease.

The prevalence of traditional and emerging cardiovascular disease risk factors was determined by the proportion of the sample that had the risk factor divided by the total number of individuals in the sample. Traditional risk factors of physical inactivity, tobacco use, and family history of CVD were assessed by self-report using the 7-Day PAR Questionnaire. The most common traditional risk factor identified was physical inactivity. A vast majority of our study population $(n=35,72.92 \%)$ did not meet 
Table 4.1 Subjects' Demographic Characteristics

Variable

Marital Status

Married

Divorced

Never married

Separated

Other

Education Level

Some high school

High school/GED

2-year college

4-year college

Graduate school

\section{Employment Status}

Unemployed

Student

Part-time

Full time

Home maker

\section{Household Income}

$<\$ 25,000$

$<\$ 50,000$

$<\$ 75,000$

$>\$ 100,000$

Illicit Drug Use

Marijuana

Past Medical History

Hypertension

Diabetes
Total Sample

$$
\text { n }(\%)
$$

$13(27.08 \%)$

$1(2.08 \%)$

$30(62.5 \%)$

$3(6.25 \%)$

$1(2.08 \%)$

$3(6.25 \%)$

$14(29.17 \%)$

$15(31.25 \%)$

$8(16.67 \%)$

$8(16.67 \%)$

$14(29.17 \%)$

$2(4.17 \%)$

$3(6.25 \%)$

$27(56.25 \%)$

$2(4.17 \%)$

$20(41.67 \%)$

$16(33.33 \%)$

$5(10.42 \%)$

$7(14.58 \%)$

$5(10.42 \%)$

$2(4.17 \%)$

$2(4.17 \%)$ 
recommended guidelines of at least 30 minutes of moderate intensity physical activity on 5 or more days of the week (CDC, 1999). Twenty-eight $(58.3 \%)$ women reported a positive family history of CVD. Positive family history of CVD was defined as a history of myocardial infarction, coronary artery disease, congestive heart failure or cerebrovascular accident in a first-degree relative or grandparent (Bertuzzi, Negri, Tavani, \& La Vecchia, 2003). Another traditional CVD risk factor that was prevalent in our study population was obesity $(n=27,56.25 \%)$. Twelve $(25 \%)$ of the women reported current tobacco use; however, the amount of tobacco use was not elicited. For the other traditional risk factors, normative values were used to determine whether an individual was classified as having the risk factor. The prevalence of traditional risk factors is summarized in Table 4.2.

Study participants were screened for emerging CVD risk factors (Table 4.3). Our results showed that all the women $(\mathrm{N}=48)$ had a QUICKI score of $<0.3$, which is suggestive of insulin resistance (Velasquez-Mieyer, Cowan, Neira, \& Tylavsky, 2008). Twenty-two (45.8\%) of the participants had both elevated high sensitivity C-reactive protein (hs-CRP $>2 \mathrm{mg} / \mathrm{dl}$ ) and Plasminogen Activator Inhibitor-1 (PAI-1 > $28.2 \mathrm{ng} / \mathrm{ml}$ ) levels. Fifty-six percent $(n=27)$ of the participants had an abnormal fibrinogen $(>350$ $\mathrm{mg} / \mathrm{dl})$ level. Three participants $(6.15 \%)$ had an elevated E-selectin level $(>79.2 \mathrm{ng} / \mathrm{ml})$, while $4.17 \%(\mathrm{n}=2)$ had elevated sICAM level $(>320 \mathrm{ng} / \mathrm{ml})$. Normative values have not yet been determined for PAI-1, E-selectin, sICAM, and QUICKI. Cutpoints used to classify individuals as having the risk factor were based on research studies.

Descriptive statistics were used to derive information regarding the data distribution of the study sample. This statistical procedure provided information regarding the characteristics of the study population such as measures of central tendency (mean, median) and variability (standard deviation, range). Table 4.4 shows a summary of the descriptive statistics for the traditional and emerging cardiovascular risk factors for the entire sample.

\section{Primary Aim Two}

Examine the relationship between BMI, physical activity levels, and other traditional and emerging cardiovascular disease risk factors in African American women.

Correlation analyses were computed to determine the interrelationship among the study variables (BMI, physical activity levels [kilo calorie per week, hours of moderate weekly physical activity, days per week of moderate physical activity], and traditional [systolic and diastolic blood pressure, fasting blood glucose, total cholesterol, high density lipoprotein cholesterol (HDL-c), low density lipoprotein cholesterol (LDL-c), triglycerides] and emerging cardiovascular risk factors [high sensitivity C-reactive protein (hs-CRP), fibrinogen, E-selectin, soluble Intercellular Adhesion Molecule-1 (sICAM-1), QUICKI, and Plasminogen Activator Inhibitor-1, (PAI-1)]. Spearman Rho correlation coefficient (r) was used to approximate the magnitude and direction of relationships among the continuous variables while Kendall Tau's were used to examine the relationships among dichotomous variables and continuous variables. Graphic plots 
Table 4.2 Prevalence of Traditional Cardiovascular Disease Risk Factors $(\mathrm{N}=48)$

Cardiovascular Disease Risk Factors

Prevalence Rates

n $(\%)$

Diabetes

$2(4.17 \%)$

Family history of CVD

$28(58.3 \%)$

Tobacco use

$12(25 \%)$

Hypertension

$2(4.17 \%)$

Elevated total cholesterol

$9(18.75 \%)$

High density lipoprotein cholesterol (HDL-c)

$18(37.5 \%)$

Low density lipoprotein cholesterol (LDL-c)

$8(16.7 \%)$

Elevated triglycerides

$3(6.25 \%)$

Physical inactivity ( $<$ 5days of physical activity/week) 35 (72.92\%)

Obesity

$27(56.25 \%)$ 
Table 4.3 Prevalence Rates of Emerging Cardiovascular Disease Risk Factors $(\mathrm{N}=48)$

$\begin{array}{ll}\text { Cardiovascular Disease Risk Factors } & \text { Rates } \\ \mathrm{n}(\%)\end{array}$

High sensitivity C-reactive protein (hs-CRP)

$22(45.8 \%)$

Fibrinogen*

$27(56.25 \%)$

Quantitative Insulin Sensitivity Check Index

$48(100 \%)$

(QUICKI)*

Plasminogen Activator Inhibitor-1 (PAI-1)**

$22(45.8 \%)$

E-selectin**

$3(6.15 \%)$

Soluble Intercellular Adhesion Molecule-1

(sICAM-1)*

$2(7.41 \%)$

* $\mathrm{N}=47$ due to technical difficulties. $* *$ Indicates that the norms for these emerging risk factors are not yet established. Values used are from reported research data. 
Table 4.4 Descriptive Statistics of Traditional and Emerging Cardiovascular Risk Factors

\begin{tabular}{|c|c|c|c|c|}
\hline $\begin{array}{l}\text { CVD Risk } \\
\text { Factors }\end{array}$ & Median & Mean & $\begin{array}{l}\text { Std Dev } \\
( \pm \text { SD })\end{array}$ & $\begin{array}{l}\text { Range } \\
\text { Min, Max }\end{array}$ \\
\hline $\begin{array}{l}\text { Average } \\
\text { systolic blood } \\
\text { pressure } \\
(\mathrm{mmHg})\end{array}$ & 114.50 & 114.96 & 13.69 & $90.00,158.00$ \\
\hline $\begin{array}{l}\text { Average } \\
\text { diastolic blood } \\
\text { pressure } \\
(\mathrm{mmHg})\end{array}$ & 72.00 & 72.82 & 9.25 & $56.50,97.00$ \\
\hline KCAL/week & 258.35 & 277.32 & 58.45 & $215.60,504.35$ \\
\hline $\begin{array}{l}\text { Hours of mod- } \\
\text { erate physical } \\
\text { activity/week }\end{array}$ & 13.15 & 21.11 & 20.71 & $0.00,73.00$ \\
\hline $\begin{array}{l}\text { Days per week } \\
\text { of moderate } \\
\text { physical activity }\end{array}$ & 3.00 & 3.21 & 2.18 & $0.00,7.00$ \\
\hline $\begin{array}{l}\text { Glucose } \\
(\mathrm{mg} / \mathrm{dl})\end{array}$ & 87.00 & 95.52 & 54.51 & $54.00,439.00$ \\
\hline $\begin{array}{l}\text { Total choles- } \\
\text { terol }(\mathrm{mg} / \mathrm{dl})\end{array}$ & 176.50 & 177.15 & 28.68 & $108.00,243.00$ \\
\hline HDL-c(mg/dl) & 52.00 & 52.85 & 13.92 & $27.00,100.00$ \\
\hline LDL-c(mg/dl) & 111.50 & 108.02 & 24.29 & $51.00,160.00$ \\
\hline $\begin{array}{l}\text { Triglycerides } \\
(\mathrm{mg} / \mathrm{dl})\end{array}$ & 62.00 & 84.02 & 74.53 & $38.00,532.00$ \\
\hline QUICKI & 0.26 & 0.26 & 0.01 & $0.19,0.29$ \\
\hline $\begin{array}{l}\text { Hs-CRP } \\
(\mathrm{mg} / \mathrm{L})\end{array}$ & 1.85 & 6.66 & 14.34 & $0.10,80.80$ \\
\hline
\end{tabular}


Table 4.4 (continued)

\begin{tabular}{lcccl}
$\begin{array}{l}\text { CVD Risk } \\
\text { Factors }\end{array}$ & Median & Mean & $\begin{array}{l}\text { Std Dev } \\
( \pm \mathrm{SD})\end{array}$ & $\begin{array}{l}\text { Range } \\
\text { Min, Max }\end{array}$ \\
\hline $\begin{array}{l}\text { Fibrinogen } \\
(\mathrm{mg} / \mathrm{dl})\end{array}$ & 387.69 & 381.74 & 135.94 & $14.28,700.49$ \\
$\begin{array}{l}\text { E-selectin } \\
(\mathrm{ng} / \mathrm{ml})\end{array}$ & 23.03 & 27.46 & 23.36 & $4.00,115.24$ \\
$\begin{array}{l}\text { sICAM-1 } \\
(\mathrm{ng} / \mathrm{ml})\end{array}$ & 80.31 & 105.02 & 96.06 & $2.91,515.95$ \\
$\begin{array}{l}\text { PAI-1 } \\
(\mathrm{ng} / \mathrm{ml})\end{array}$ & 23.79 & 32.69 & 27.57 & $0.37,104.17$ \\
\hline
\end{tabular}

Kcal/week = kilocalorie per week, HDL-c = high density lipoprotein cholesterol, LDL-c $=$ low density lipoprotein cholesterol, QUICKI = quantitative insulinsensitivity check index, hs-CRP $=$ high sensitivity C-reactive protein.

were performed to facilitate the visualization of the relationship between the variables of interest and to determine their linearity. Table 4.5 provides a summary of the correlational analyses performed among these variables.

Our results showed a significant positive relationship between BMI and systolic blood pressure $(r=0.67 ; p<0.0001)$. This finding suggest that the higher the BMI, the higher the systolic blood pressure. The correlation between kilocalories of energy expended per week and systolic blood pressure $(r=-0.03 ; p=0.85)$ and between hours spent in moderate physical activity per week and systolic blood pressure was $(\mathrm{r}=-0.11 ; \mathrm{p}$ $=0.45$ ). The association between the number of days spent in moderate physical activity and systolic blood pressure was $(r=0.3 ; p=0.84)$. These were not statistically significant between the groups.

A significant relationship was noted between BMI and diastolic blood pressure ( $\mathrm{r}$ $=0.61 ; \mathrm{p}<0.0001)$. Just like for systolic blood pressure, the higher the BMI, the higher the diastolic blood pressure. There was a non significant association between kilocalories expended per week and diastolic blood pressure was $(r=-0.18 ; p=0.21)$ and between hours spent in moderate physical activity per week and diastolic blood pressure $(\mathrm{r}=$ $-0.09 ; p=0.54)$. The association between the number of days participants spent in moderate physical activity and diastolic blood pressure $(r=-0.24 ; p=0.09)$ was also not significant. 
Table 4.5 Relationship among BMI, Physical Activity, and CVD Risk Factors ( $=48)$

CVD

Risk

Factors
Hours of

blood

pressure

Diastolic

blood

pressure

Fasting

blood

glucose

QUICKI*

Total

cholesterol

High density

Lipoprotein

cholesterol

Low density

lipoprotein

cholesterol

Triglycerides

$$
\begin{aligned}
& r=0.30 \\
& p=0.04
\end{aligned}
$$

$\mathrm{r}=0.51$

$\mathrm{p}=0.0002$

sensitivity

CRP

Fibrinogen kcal/wk

BMI

$r=0.67$

$\mathrm{p}<.0001$

$$
\begin{aligned}
& \mathrm{r}=-0.12 \\
& \mathrm{p}=0.41
\end{aligned}
$$$$
\mathrm{r}=-0.35
$$$$
\mathrm{p}=0.01
$$

$\mathrm{r}=-0.04$

$\mathrm{p}=0.76$

$\mathrm{r}=0.28$

$\mathrm{p}=0.05$

$\mathrm{r}=0.21$

$\mathrm{p}=0.14$

$\mathrm{r}=0.24$

$\mathrm{p}=0.11$

$p=0.13$

$\mathrm{p}=0.13$

$\mathrm{r}=0.17$

$\mathrm{p}=0.25$

$r=-0.09$

$\mathrm{p}=0.54$

$r=-0.24$

$\mathrm{p}=0.09$

$r=0.03$

$\mathrm{p}=0.84$

Moderate

Activity Wkly 
Table 4.5 (continued)

CVD

Risk

Factors
BMI $\quad \mathrm{kcal} / \mathrm{wk}$
Hours of

Moderate Activity Wkly
Days of at Least

Moderate

Activity Wkly

$\begin{array}{lllll}\text { E-selectin } & \mathrm{r}=0.21 & \mathrm{r}=-0.24 & \mathrm{r}=-0.23 & \mathrm{r}=0.22 \\ & \mathrm{p}=0.15 & \mathrm{p}=0.10 & \mathrm{p}=0.12 & \mathrm{p}=0.13 \\ \text { sICAM-1 } & \mathrm{r}=0.33 & \mathrm{r}=-0.27 & \mathrm{r}=-0.25 & \mathrm{r}=0.31 \\ & \mathrm{p}=0.02 & \mathrm{p}=0.06 & \mathrm{p}=0.09 & \mathrm{p}=0.03 \\ \text { PAI-1 } & \mathrm{r}=0.21 & \mathrm{r}=-0.18 & \mathrm{r}=-0.19 & \mathrm{r}=0.13 \\ & \mathrm{P}=0.15 & \mathrm{p}=0.23 & \mathrm{p}=0.21 & \mathrm{p}=0.36 \\ \text { Tobacco use } & \mathrm{r}=0.05 & \mathrm{r}=-0.15 & \mathrm{r}=-0.01 & \mathrm{r}=-0.25 \\ & \mathrm{p}=0.65 & \mathrm{p}=0.22 & \mathrm{p}=0.96 & \mathrm{p}=0.05 \\ \text { Family hx } & \mathrm{r}=-0.01 & \mathrm{r}=-0.08 & \mathrm{r}=0.02 & \mathrm{r}=-0.02 \\ \text { of CVD } & \mathrm{p}=0.93 & \mathrm{p}=0.52 & \mathrm{p}=0.84 & \mathrm{p}=0.87 \\ & & & & \\ \text { HTN } & \mathrm{r}=0.21 & \mathrm{r}=0.09 & \mathrm{r}=-0.05 & \mathrm{r}=0.18 \\ & \mathrm{p}=0.08 & \mathrm{p}=0.41 & \mathrm{p}=0.67 & \mathrm{p}=0.16\end{array}$

*N $=47$ due to technical difficulties. QUICKI = quantitative insulin-sensitivity check index, sICAM-1 = soluble intercellular adhesion molecule-1, PAI-1 = plasminogen activator inhibitor-1, Family hx of CVD = family history of cardiovascular disease, $\mathrm{HTN}=$ hypertension. 
The results of our analyses showed a negative significant association between BMI and HDL-cholesterol $(r=-0.35 ; \mathrm{p}=0.01)$. This means that the higher the BMI, the lower the HDL-cholesterol levels. A non significant association was noted between kilocalories expended per week and HDL-cholesterol $(r=0.24 ; \mathrm{p}=0.11)$ and also between the hours spent in moderate physical activity and HDL-cholesterol $(r=0.22 ; \mathrm{p}=$ $0.13)$. A non- significant association was noted between the number of days spent in moderate physical activity and HDL-cholesterol $(\mathrm{r}=-0.16 ; \mathrm{p}=0.30)$.

Our findings showed a non significant association between BMI and LDLcholesterol $(r=-0.04 ; p=0.76)$. There was a significant association between the kilocalories of energy expended per week and LDL-cholesterol $(r=0.28 ; p=0.05)$. This finding is surprising as it tends to suggest that the participants who exerted more kilocalories of energy per week had higher LDL-cholesterol. A non significant association was observed between hours of moderate physical activity per week and LDL-cholesterol $(\mathrm{r}=0.27 ; \mathrm{p}=0.07)$ as well as between days spent in moderate physical activity and LDL-cholesterol $(\mathrm{r}=0.12 ; \mathrm{p}=0.43)$.

We also observed a significant correlation between BMI and hs-CRP $(r=0.51$; $p=0.0002)$. This finding suggests that a higher BMI was positively associated with a higher hs-CRP level. There was a non significant association between kilocalories of energy expended per week and hs-CRP $(\mathrm{r}=-0.12 ; \mathrm{p}=0.41)$ as well as between hours spent in moderate physical activity per week and hs-CRP $(r=-0.22 ; p=0.14)$. The association between days spent in moderate physical activity per week and hs-CRP was non significant $(\mathrm{r}=0.12 ; \mathrm{p}=0.41)$.

The results of our analyses showed a significant association between BMI and fibrinogen $(r=0.50 ; p=0.0004)$. Again, this indicates that a higher BMI was associated with a higher fibrinogen level. A non significant association was noted between kilocalories of energy expended per week and fibrinogen $(r=0.01 ; p=0.92)$ and between hours spent in moderate physical activity per week and fibrinogen $(r=-0.15 ; \mathrm{p}=0.32)$ as well as a non significant association between days spent in moderate physical activity per week and fibrinogen $(r=0.06 ; p=0.67)$.

Our findings also showed a significant association between BMI and sICAM-1 $(r=0.33 ; p=0.02)$. Again, this implies that the higher the BMI, the higher the sICAM-1 level. We determined there was a non significant association between the kilocalorie expended based on body weight and SICAM-1 $(r=-0.27 ; \mathrm{p}=0.06)$ as well as between the number of hours of moderate physical activity performed weekly by individual participants and sICAM-1 level $(\mathrm{r}=-0.25 ; \mathrm{p}=0.09)$. There was a significant association between the number of days participants spent in moderate physical activity per week and sICAM-1 level $(r=0.31 ; p=0.03)$. 


\section{Primary Aim Three}

Compare traditional and emerging cardiovascular risk factors among normal weight and obese African American women.

The demographic characteristics of the normal weight and obese groups are shown in Table 4.6. Our data revealed that more women $66.67 \%(n=18)$ in the obesity group were single compared to $57.14 \%(\mathrm{n}=12)$ of the normal weight group. Forty-one percent $(n=11)$ of the obese group had a high school degree/GED or less compared to $28.6 \%(n=6)$ of the normal weight group. The study participants had comparable levels of college education $(59.26 \%, \mathrm{n}=16$ for normal weight group vs. $71.4 \%, \mathrm{n}=15$ for obese group, $\mathrm{p}=\mathrm{NS})$. About $30 \%(\mathrm{n}=8)$ of the obese group described themselves as unemployed compared to $28.6 \%(n=6)$ of the normal weight group. Seventeen $(63 \%)$ of the obese group were employed compared to $61.9 \%(n=13)$ of the normal weight group. With regards to annual household income, $85.2 \%(\mathrm{n}=23)$ of the obese group in our study reported an annual income that was $<\$ 50,000$, while $61.9 \%(\mathrm{n}=13)$ of the normal weight group reported similar income levels. Almost twice as many participants in our normal weight group $(38.1 \%, \mathrm{n}=8)$ reported an annual household income of $\$ 50,000$ to $\$ 100,000$, compared to $14.8 \%(n=4)$ of the obese group. Three $(11.11 \%)$ participants in our obese group admitted to the use of marijuana compared to $9.52 \%(n=2)$. Two of our obese participants reported a past medical history of hypertension and diabetes $(\mathrm{n}=2$, $7.41 \%$ ). Details of descriptive statistics for the normal weight and obese groups are further summarized in Table 4.7.

Since non-parametric statistics are most appropriately used when the sample sizes are small, the Mann Whitney $U$ test was used to examine the differences in cardiovascular risk factors between the normal weight and obese groups. Continuous variables measured in this analysis were systolic and diastolic blood pressure, lipid profile (total cholesterol, HDL-c, LDL-c, triglycerides), fasting plasma glucose, hours spent weekly on moderate physical activity, number of days of the week spent in moderate physical activity, QUICKI, hs-CRP, fibrinogen, E-selectin, sICAM-1, and PAI1. Values are summarized in Table 4.8.

A summary of the results of the independent sample t-test statistics procedures shown in Table 4.8 indicated that the obese weight group had higher systolic $(\mathrm{p}=$ $0.0002)$ and diastolic blood pressure $(p=0.0007)$, and lower HDL-cholesterol $(p=0.01)$ and higher triglyceride levels $(p=0.02)$ than the normal weight group. There were no statistically significant difference between groups in the levels of total cholesterol $(\mathrm{p}=$ $0.6065)$, LDL-cholesterol $(\mathrm{p}=0.8040)$, hours of moderate physical activity performed weekly $(\mathrm{p}=0.3597)$, and the number of days per week of moderate physical activity $(\mathrm{p}=$ 0.6670). A similar trend was found with the emerging cardiovascular risk factors between the two groups. The obese group had significantly higher levels of hs-CRP $(p=0.0020)$, and fibrinogen $(\mathrm{p}=0.0109)$ compared to the normal weight group. However, the difference in the levels of QUICKI $(p=0.3382)$, E-selectin $(p=0.2884), \operatorname{sICAM}(p=$ $0.0950)$, and PAI-1 ( $\mathrm{p}=0.3985)$ between the two groups was not statistically significant. 
Table 4.6 Demographic Characteristics by Weight Groups

\begin{tabular}{|c|c|c|}
\hline Variable & $\begin{array}{l}\text { Normal Weight Group } \\
(\mathrm{n}=21) \\
\mathrm{n}(\%)\end{array}$ & $\begin{array}{l}\text { Obese Group } \\
\quad(\mathrm{n}=27) \\
\mathrm{n}(\%)\end{array}$ \\
\hline \multicolumn{3}{|l|}{ Marital Status } \\
\hline Married & $7(33.33 \%)$ & $6(22.22 \%)$ \\
\hline Divorced & 0 & $1(3.70 \%)$ \\
\hline Never Married & $12(57.14 \%)$ & $18(66.67 \%)$ \\
\hline Separated & $2(9.52 \%)$ & $1(3.70 \%)$ \\
\hline Other & 0 & $1(3.70 \%)$ \\
\hline \multicolumn{3}{|l|}{ Education Level } \\
\hline Some high school & $1(4.76 \%)$ & $2(7.41 \%)$ \\
\hline High school/GED & $5(23.81 \%)$ & $9(33.33 \%)$ \\
\hline 2-year college & $5(23.81 \%)$ & $10(37.04 \%)$ \\
\hline 4-year college & $5(23.81 \%)$ & $3(11.11 \%)$ \\
\hline Graduate School & $5(23.81 \%)$ & $3(11.11 \%)$ \\
\hline \multicolumn{3}{|l|}{ Employment Status } \\
\hline Unemployed & $6(28.57 \%)$ & $8(29.63)$ \\
\hline Student & $1(4.76 \%)$ & $1(3.70 \%)$ \\
\hline Part-time & $1(4.76 \%)$ & $2(7.41 \%)$ \\
\hline Full time & $12(57.14 \%)$ & $15(55.56 \%)$ \\
\hline Home maker & $1(4.76 \%)$ & $1(3.70 \%)$ \\
\hline \multicolumn{3}{|l|}{ Household Income } \\
\hline$<\$ 25,000$ & $8(38.10 \%)$ & $12(44.44 \%)$ \\
\hline$>\$ 25,000-\$ 50,000$ & $5(23.81 \%)$ & $11(40.74 \%)$ \\
\hline$>\$ 50,000-\$ 75,000$ & $3(14.29 \%)$ & $2(7.41 \%)$ \\
\hline$>\$ 75,000-\$ 100,000$ & $5(23.81 \%)$ & $2(7.41 \%)$ \\
\hline \multicolumn{3}{|l|}{ Illicit Drug Use } \\
\hline Marijuana & $2(9.52 \%)$ & $3(11.11 \%)$ \\
\hline \multicolumn{3}{|l|}{ Past Medical History } \\
\hline Hypertension & 0 & $2(7.41 \%)$ \\
\hline Diabetes & 0 & $2(7.41 \%)$ \\
\hline
\end{tabular}


Table 4.7 Comparison of Means of the Traditional and Emerging Cardiovascular Risk Factors among Normal Weight and Obese African American Women

\begin{tabular}{|c|c|c|}
\hline Risk Factors & $\begin{array}{l}\text { Normal Weight } \\
(\mathrm{n}=21) \\
\mathrm{M}( \pm \mathrm{SD})\end{array}$ & $\begin{array}{l}\text { Obese Group } \\
(\mathrm{n}=27) \\
\mathrm{M}( \pm \mathrm{SD})\end{array}$ \\
\hline ASBP mmHg & $106.12(9.98)$ & $121.83(12.25)$ \\
\hline ADBP mmHg & $67.36(6.61)$ & $77.07(8.84)$ \\
\hline $\mathrm{TC} \mathrm{mg} / \mathrm{dl}$ & $178.19(25.04)$ & $176.33(31.67)$ \\
\hline HDL-c mg/dl & $57.86(11.34)$ & $48.96(14.67)$ \\
\hline LDL-c mg/dl & $108.19(23.84)$ & $107.89(25.08)$ \\
\hline Trig mg/dl & $66.76(33.98)$ & $97.44(93.41)$ \\
\hline Glucose mg/dl & $85.33(10.07)$ & $103.44(71.72)$ \\
\hline Hrs of moderate wkly activity & $24.02(22.07)$ & $18.86(19.71)$ \\
\hline Days/wk of moderate activity & $3.43(2.60)$ & $3.04(1.83)$ \\
\hline QUICKI & $0.26(0.01)$ & $0.26(0.02)$ \\
\hline Hs-CRP mg/l & $1.88(2.84)$ & $10.38(18.25)$ \\
\hline Fibrinogen mg/dl & $327.68(126.12)$ & $425.41(129.77)$ \\
\hline E-selectin ng/ml & $25.01(25.20)$ & $29.37(22.13)$ \\
\hline sICAM -1 ng/ml & $77.86(42.98)$ & $126.15(119.17)$ \\
\hline PAI-1 ng/ml & $27.96(22.99)$ & $36.37(30.59)$ \\
\hline \multicolumn{3}{|c|}{$\begin{array}{l}\mathrm{M}( \pm \mathrm{SD})=\text { Mean }(\text { Standard deviation }), \mathrm{ASBP}=\text { average systolic blood pressure, } \mathrm{ADBP}= \\
\text { average diastolic blood pressure, TC }=\text { total cholesterol, HDL-c }=\text { high density } \\
\text { lipoprotein cholesterol, LDL-c }=\text { low density lipoprotein cholesterol, Trig = triglycerides, } \\
\text { QUICKI = quantitative insulin-sensitivity check index, hs-CRP = high sensitivity C- } \\
\text { reactive protein, sICAM-1 = soluble intercellular adhesion molecule-1, PAI-1 = } \\
\text { plasminogen activator inhibitor- } 1 .\end{array}$} \\
\hline
\end{tabular}


Table 4.8 Comparison of Traditional and Emerging Cardiovascular Risk Factors among Normal Weight and Obese African American Women

\begin{tabular}{|c|c|c|c|c|}
\hline Risk Factors & $\begin{array}{l}\text { Normal Weight } \\
\quad(\mathrm{n}=21) \\
\mathrm{M}(25 \mathrm{th}, 75 \mathrm{th}) *\end{array}$ & $\begin{array}{l}\text { Obese Group } \\
\qquad(\mathrm{n}=27) \\
M(25 \mathrm{th}, 75 \mathrm{th})\end{array}$ & t-Test & P-Value \\
\hline ASBP & $103.5(99.5,110)$ & $120.5(115,128.5)$ & 16.807 & 0.0002 \\
\hline ADBP & $67.5(63,72)$ & $74.5(70,85)$ & 13.103 & 0.0007 \\
\hline $\mathrm{TC}$ & $182(163,197)$ & $175(163,193)$ & 0.2811 & 0.6065 \\
\hline HDL-c & $58(52,65)$ & $49(38,57)$ & 7.2584 & 0.0100 \\
\hline LDL-c & $114(89,126)$ & $111(90,112)$ & 0.0676 & 0.8040 \\
\hline Trig & $56(52,66)$ & $71(59,97)$ & 5.6166 & 0.0225 \\
\hline Glucose & $83(81,90)$ & $89(80,102)$ & 0.9563 & 0.3382 \\
\hline $\begin{array}{l}\text { Hrs of moderate } \\
\text { wkly activity }\end{array}$ & $18.0(3.0,73.0)$ & $13.0(0.0,69.0)$ & 0.8750 & 0.3597 \\
\hline $\begin{array}{l}\text { Days/wk of moderate } \\
\text { activity }\end{array}$ & $3.0(0.0,7.0)$ & $3.0(0.0,7.0)$ & 0.1967 & 0.6670 \\
\hline QUICKI & $.26(.256, .262)$ & $.256(.245, .263)$ & 0.9563 & 0.3382 \\
\hline Hs-CRP & $0.7(0.3,2.0)$ & $4.1(1.3,10.4)$ & 10.800 & 0.0020 \\
\hline Fibrinogen & $332(296,388)$ & $441(354.5,481)$ & 7.0988 & 0.0109 \\
\hline E-selectin & $17.5(13.0,28.2)$ & $24(15.5,39.4)$ & 1.1757 & 0.2884 \\
\hline sICAM-1 & $68.6(54.8,89.4)$ & $95.3(63.8,150.1)$ & 2.9397 & 0.0950 \\
\hline PAI-1 & $22.4(13.8,34.2)$ & $25.1(11.4,51.6)$ & 0.7439 & 0.3985 \\
\hline \multicolumn{5}{|c|}{$\begin{array}{l}\text { *M }=\text { Median (Interquatile range), } \mathrm{ASBP}=\text { average systolic blood pressure, } \mathrm{ADBP}= \\
\text { average diastolic blood pressure, TC }=\text { total cholesterol, HDL-c }=\text { high density } \\
\text { lipoprotein cholesterol, LDL-c }=\text { low density lipoprotein cholesterol, Trig = triglycerides, } \\
\text { QUICKI = quantitative insulin-sensitivity check index, hs-CRP = high sensitivity C- } \\
\text { reactive protein, sICAM-1 = soluble intercellular adhesion molecule-1, PAI-1 = } \\
\text { plasminogen activator inhibitor-1. }\end{array}$} \\
\hline
\end{tabular}


Chi-square analysis provided an assessment of observed differences between the proportions of cardiovascular risk factors in the two groups (normal weight and obese) to ascertain their statistical significance. When there were less than 5 counts in a cell, Fisher exact test was used as a more conservative measure. Table 4.9 depicts the frequency of traditional and emerging cardiovascular risk factors in the normal weight and obese groups. The normal weight and obese groups had a similar frequency of traditional risk factors of diabetes, family history of cardiovascular disease, tobacco use, hypertension, physical inactivity, and elevated total, LDL, and triglyceride levels. Low HDL-c was more common in the obese group compared to the normal weight group.

With regards to the emerging cardiovascular risk factors, our results indicated that the obese group exhibited a higher prevalence of elevated hs-CRP and fibrinogen markers compared to the normal weight group (Table 4.9). The frequency of elevated PAI-1 was similar between the normal weight and obese groups (42.86\% vs, 48.15\%). Chi-square analysis for E-selectin, sICAM-1, and QUICKI was not performed because the norms for these novel risk factors have not yet been established. For the purposes of classification, the cutpoint for these values was E-selectin $>79.2 \mathrm{ng} / \mathrm{ml}$, sICAM-1 $>320 \mathrm{ng} / \mathrm{ml}$, and QUICKI $<0.30 \mathrm{mg} / \mathrm{dl}$. Abnormal E-selectin and sICAM-1 were infrequent; however, all participants in both groups exhibited insulin resistance, as defined by a QUICKI level < 0.30 .

\section{Average Number of Risk Factors by Weight Group}

The average number of risk factors an individual participant had was computed by adding the number of risk factors divided by the sample population. The average number of risk factors was 5.7 for the whole group. Compared to the normal weight group, the obese group had significantly more risk factors (6.9. vs. 4.1, $\mathrm{p}<0.05)$.

\section{Average Number of Risk Factors by Physical Activity}

We further determined the average number of risk factors based on physical activity status by weight group and compared the groups. This was determined by the total number of risk factors within each weight category divided by the total number in their respective physical activity status categories. The obese participants who were physically active had an average of 6.6 risk factors compared to 6.9 in those who were inactive. Similarly, the normal weight participants who were active had an average of 3.9 risk factors compared to 4.3 in those who were physically inactive. While there was no statistically significant difference within weight groups, we noted a statistically significant difference between weight groups $(\mathrm{p}<0.05)$. 
Table 4.9 Differences in Frequency of Cardiovascular Risk Factors between Weight Groups

\begin{tabular}{|c|c|c|c|c|}
\hline Risk Factors & $\begin{array}{c}\text { Normal Weight } \\
\mathrm{n}(\%)\end{array}$ & $\begin{array}{c}\text { Obese Group } \\
n(\%)\end{array}$ & $\begin{array}{c}\text { Chi-Square } \\
\chi 2\end{array}$ & P-Value \\
\hline Diabetes & 0 & $2(7.41 \%)$ & 1.8847 & 0.3897 \\
\hline Fm Hx CVD & $13(61.90 \%)$ & $15(55.56 \%)$ & 0.1959 & 0.6580 \\
\hline Tobacco use & $5(23.81 \%)$ & $7(25.93 \%)$ & 0.0282 & 0.8666 \\
\hline HTN & 0 & $2(7.41 \%)$ & 11.7188 & 0.0029 \\
\hline $\mathrm{TC}$ & $4(19.05 \%)$ & $5(18.52 \%)$ & 0.0022 & 0.9628 \\
\hline HDL-c & $4(19.05 \%)$ & $14(51.85 \%)$ & 5.4236 & 0.0199 \\
\hline LDL-c & $4(19.05 \%)$ & $4(14.81 \%)$ & 0.1524 & 0.6963 \\
\hline Trig & $9(42.86 \%)$ & $2(7.41 \%)$ & 0.1411 & 0.7072 \\
\hline $\begin{array}{l}\text { Physical inactivity } \\
(<5 \text { days of } \\
\text { physical activity/wk) }\end{array}$ & $13(61.90 \%)$ & $22(81.48 \%)$ & 2.2924 & 0.1300 \\
\hline Hs-CRP & $5(23.81 \%)$ & $17(62.96 \%)$ & 7.2940 & 0.0069 \\
\hline Fibrinogen & $7(33.33 \%)$ & $20(76.92 \%)$ & 9.0295 & 0.0027 \\
\hline PAI-1 & $9(42.86 \%)$ & $13(48.15 \%)$ & 0.1332 & 0.7151 \\
\hline E-selectin & $1(4.76 \%)$ & $2(7.41 \%)$ & & \\
\hline sICAM-1 & 0 & $2(7.41 \%)$ & & \\
\hline QUICKI & $21(100 \%)$ & $27(100 \%)$ & & \\
\hline
\end{tabular}

Fm Hx CVD = family history of cardiovascular disease, $\mathrm{HTN}=$ hypertension, $\mathrm{TC}=$ total cholesterol, HDL-c = high density lipoprotein cholesterol, LDL-c = low density lipoprotein cholesterol, Trig = triglyceride, hs-CRP $=$ high sensitivity $\mathrm{C}=$ reactive protein, PAI-1 = plasminogen activator inhibitor-1, sICAM-1 = soluble intercellular adhesion molecule-1, QUICKI = quantitative insulin-sensitivity check index. $\mathrm{n}(\%)$ : values represent abnormal levels of the risk factors. 


\section{CHAPTER 5. DISCUSSION AND IMPLICATIONS}

\section{Discussion}

Cardiovascular disease and obesity are major public health concerns of our times. Women bear the highest prevalence of morbidity and mortality from cardiovascular disease and African American women are the most affected. While there have been multiple focused studies on either cardiovascular disease or obesity, only limited studies have explored the interaction between obesity and cardiovascular disease risk factors in women and much fewer studies have specifically looked at this unique population of African American women. This study sought to explore the relationship between obesity and the traditional and emerging cardiovascular risk factors in young African American women with no previous history of cardiovascular disease. This chapter includes a discussion of significant findings and compares study results to existing research. Study limitations and recommendations for research, theory, and practice are also discussed.

The results show that both traditional and emerging cardiovascular disease risk factors were more prevalent among obese than normal weight subjects. This difference was statistically significant with regards to HDL-cholesterol $(\mathrm{p}=0.02), \mathrm{hs}-\mathrm{CRP}(\mathrm{p}=$ $0.01)$, and Fibrinogen $(p=0.002)$. These findings are similar to the results of the MultiEthnic Study of Atherosclerosis, which examined the relation of obesity in older individuals 45 to 84 years old (Bild et al., 2005). However, that study looked at only traditional risk factors. There are other studies that have similarly shown a higher prevalence of emerging cardiovascular risk factors among obese young adults (Appel, Harrell, \& Davenport, 2005; Santos, Lopes, Guimaraes, \& Barros, 2005). These findings carry implications for clinical practice: Obese adults without clinical cardiovascular disease may already have a clustering of both traditional and emerging cardiovascular risk factors. Institution of early and aggressive preventive measures in this population could delay or arrest progression to clinical cardiovascular disease.

The knowledge gained by conducting this descriptive correlation analysis could provide health care providers with the tools they need to educate and inform women about their risks for cardiovascular disease and help them develop strategies for disease prevention. To reduce the high morbidity and mortality of cardiovascular disease among African American women, regular cardiovascular disease screening and intervention should be started in early adulthood. Contrary to prevailing belief that heart disease is rare among women and highly unlikely in premenopausal women, health care providers should be reeducated on the need for treating cardiovascular risk in young adults.

\section{Prevalence of Traditional and Emerging Cardiovascular Risk Factors}

The first research aim of this study focused on describing the prevalence of traditional and emerging cardiovascular risk factors in African American women ages 18 to 45 who have no known history of cardiovascular disease. The results of our study showed that this sample of African American women had a high prevalence of both 
traditional and emerging cardiovascular risk factors. They presented with varying degrees of the traditional cardiovascular risk factors with physical inactivity (72.92\%), family history of cardiovascular disease (58.3\%), low HDL-cholesterol levels $(37.5 \%)$, and smoking $(25 \%)$ being highly prevalent. This finding is consistent with the rate reported by De, Searles, and Haddad (2002) in their study on the prevalence of cardiac risk factors in 187 women 45 years of age and younger undergoing angiography for evaluation of undiagnosed chest pain, a high-risk group. The most prevalent risk factor they found for all study participants was a family history of premature coronary artery disease (67\%), followed by smoking (55\%), and dyslipidemia (55\%). They further reported that younger women with coronary artery disease had a higher prevalence of dyslipidemia, diabetes, and smoking than those without coronary artery disease. Gurevitz, Jonas, Boyko, Rabinowitz, and Reicher-Reiss (2000), in a similar study of women aged 50 and under, observed that the most prominent risk factor was hyperlipidemia $(60 \%)$, followed by a family history of coronary artery disease $(44 \%)$, systemic hypertension $(40 \%)$, cigarette smoking $(31 \%)$, postmenopausal state $(23 \%)$, and diabetes mellitus $(21 \%)$, with hyperlipidemia and postmenopausal state more prevalent in those with significant coronary artery disease.

Compared to the American Heart Association (2008) Statistical Fact Sheets of women of all ages, our study showed a higher prevalence of low HDL-cholesterol in our selected population $(37.5 \%, 6.9 \%)$. Hypertension prevalence was much lower among study participants than what was reported by the American Heart Association (4.17\% vs. $46.6 \%)$, while a difference was also noted in the rate of tobacco use (25\% vs. $17.3 \%)$. The Centers for Disease Control and Prevention analyzed data from the Behavioral Risk Factor Surveillance System (BRFSS) for the period 1994-2004 and reported a prevalence rate of leisure time physical inactivity among non-Hispanic black women at a rate of $33.9 \%$ while $72.92 \%$ of participants in this study reported they did not engage in moderate physical activity five or more days per week.

With regards to the prevalence rates of the emerging cardiovascular risk factors, a literature search revealed that prevalence rates were not routinely reported. This is not surprising given the newness of most of these biomarkers and the lack of large studies to determine normative values., However, the literature does indicate that biomarkers such as hs-CRP, Fibrinogen, insulin resistance, PAI-1, E-selectin and sICAM-1 vary in their levels among people of diverse ethnic identities particularly African Americans (Albert \& Ridker, 2006; Sumner et al., 2008).

Coronary artery inflammation is involved in all stages of atherosclerotic plaque formation. Markers of systemic inflammation can predict future cardiovascular events in healthy persons and patients with coronary artery disease (Wilson et al., 1998). Available literature indicates that measurement of certain inflammatory markers can help identify high-risk patients, monitor disease activity, and provide a therapeutic guide for reducing the inflammatory component of the disease (Rosenson \& Koenig, 2003). 


\section{Relationships between Cardiovascular Risk Factors}

The second aim of the study was to examine the relationship between BMI, physical activity levels, and other traditional and emerging cardiovascular disease risk factors in African American women. In the adult population, obesity is defined as a body mass index (BMI) of $30 \mathrm{~kg} / \mathrm{m}^{2}$ or higher (Flegal et al., 2002). BMI was calculated by using the Center for Disease Control formula expressed as weight in kilograms divided by the square of height in meters. Obesity and physical inactivity have long been associated with increased risk of cardiovascular disease and are associated with traditional risk factors such as diabetes, hypertension, and hyperlipidemia (Fung et al., 2001) and these risk factors contribute to thousands of preventable deaths in the United States (CDC, 1999). Women who are sedentary are twice as likely to develop cardiovascular disease as women who engage in physical activity (CDC, 2007a).

\section{Relationships between BMI, Physical Activity, and Blood Pressure}

The findings from our study showed a positive association between BMI and blood pressure as well as physical activity and blood pressure. The results of our study showed a significant positive relationship between BMI and systolic blood pressure $(\mathrm{r}=$ $0.67, \mathrm{p}<0.0001)$. Our study also showed a significant positive relation between BMI and diastolic blood pressure $(r=0.61, p=<0.0001)$. A negative, non-significant association was also noted between days spent per week in moderate levels of physical activity and diastolic blood pressure $(\mathrm{r}=-0.24, \mathrm{p}=0.09)$. These findings suggest that high BMI and low physical activity levels (means $<5$ days of moderate physical activity weekly) tended to result in elevated systolic and diastolic blood pressure levels. This finding is consistent with reports from the American Heart Association, which suggests that excess body weight increases your risk of high blood pressure. Scherrer et al. (1991) noted that a 20pound increase in body weight is associated with a $3.0-\mathrm{mmHg}$ higher systolic and 2.3$\mathrm{mmHg}$ higher diastolic blood pressure. This translates into an estimated $12 \%$ increased risk for coronary heart disease in obese individuals (Chobanian et al., 2003).

In another study, Staffileno, Minnick, Coke, \& Hollenberg (2007) examined the effect of lifestyle physical activity on blood pressure indices in sedentary AfricanAmerican women aged 18 to 45 years with prehypertension or untreated stage 1 hypertension. They reported a significant reduction in systolic and diastolic blood pressures in women who engaged in physical activity $(r=-0.620, p=0.024)$. According to the American Heart Association, the incidence of cardiovascular risk factors such as high blood pressure, physical inactivity, and obesity are higher in African American women than in Caucasian women (AHA, 2008; Hajjar \& Kotchen, 2003).

\section{Correlations between BMI, Physical Activity, and Diabetes}

High blood glucose or diabetes is another risk factor for cardiovascular disease. The obesity epidemic has led to an emerging epidemic of type 2 diabetes, and African 
American women have been severely affected by both conditions. The results from our study showed a non-significant relationship between BMI and fasting blood glucose $(r=$ $0.12, p=0.41$ ), and between the number of days per week that participants engaged in moderate weekly physical activity and fasting blood glucose $(r=0.03, p=0.87)$. Other research studies have shown that obesity is a strong risk factor for diabetes in African American women. Krishnan, Rosenberg, Djousse, Cupples, and Palmer (2007) examined the association between BMI, abdominal obesity, and weight gain in African American women with risk of type 2 diabetes. They reported that during an 8 year follow-up period of 49,766 women from the Black Women's Health Study, 2,472 incident cases of diabetes occurred. Krishnan, Rosenberg, and Palmer (2008) further used data from 45,668 black women aged 21 to 69 years to estimate incidence rate ratios for type 2 diabetes by comparing various levels of physical activity and television watching. They found that vigorous activity was inversely associated with type 2 diabetes risk ( $\mathrm{p}<$ 0.0001 ). The associations between physical activity and diabetes risk have been assessed by a number of prospective studies and clinical trials. The results from these studies consistently indicate that regular physical activity during leisure time or daily life reduces the risk of type 2 diabetes by 15 to $60 \%$ (Qi, Hu, \& Hu, 2008).

\section{Relationships between BMI, Physical Activity, and Insulin Resistance}

There is growing knowledge regarding the effect of insulin resistance and cardiovascular health. The findings of our study show a weak negative non-significant relationship between BMI and QUICKI [used to calculate insulin sensitivity] $(\mathrm{r}=-0.12, \mathrm{p}$ $=0.41)$. Given that there was little variability in QUICKI values among participants, it is not surprising that the relationship was weak. A similar association was found between days of moderate physical activity that participants engaged in per week and insulin resistance $(r=-0.17, p=0.23)$. Published research largely agrees with our findings. Even though their study examined a cohort of urban, predominantly African American adolescents, Snitker, Le, Hager, Caballero, and Black (2007) reported an association between physical activity and body composition with insulin sensitivity in a community sample of adolescents and noted that both body composition and physical activity were independently associated with insulin sensitivity.

\section{Correlations between BMI, Physical Activity, and Lipid Levels}

Serum lipids and lipoproteins (such as HDL-cholesterol, LDL-cholesterol, total cholesterol, and triglycerides) are metabolic risk factors most closely related to atherosclerosis. The findings from this current study showed a weak relationship between total cholesterol, HDL-cholesterol, LDL-cholesterol, triglycerides and BMI and days spent in moderate physical activity per week. Statistical significance was noted between BMI and HDL-cholesterol as well as between BMI and triglycerides $(r=-0.35$, $p=0.01 ; r=0.30, p=0.04)$. This finding is consistent with what has been reported in the literature. Rodriguez-Arcos, Smith, and Waldron (2002) studied cross-sectionally the distribution and predictors of lipids and lipoproteins in elderly subjects and reported that 
African Americans had a lower total/HDL-cholesterol ratio and triglyceride levels along with higher HDL-cholesterol levels $(\mathrm{p}<0.0001)$. Body mass index was a significant independent predictor of total cholesterol (beta $=0.74, \mathrm{p}<0.001$ ). Walker, Piers, Putt, Jones, and O'Dea (1999) examined the impact of a 16-week walking program on the metabolic risk profile of postmenopausal women. Even though the age category varies from our study population, the findings are noteworthy. In the sample of 153 women, they reported that increased physical activity promoted weight loss, reduced blood pressure, decreased plasma triglyceride, total cholesterol, and LDL-cholesterol, but increased HDL cholesterol concentrations $(p<0.0001)$.

\section{Relationships between BMI, Physical Activity, and Tobacco Use}

Tobacco use is another risk factor examined in our study. Some research indicates that smoking behavior is related to other health behaviors, such as sedentary lifestyle, body weight, and consumption of fast food. Larsson, Story, Perry, NeumarkSztainer, and Hannan (2007) examined how smoking may be related to dietary intake of key nutrients, consumption of fast food, sedentary lifestyle, or weight status. They reported that smoking frequency was inversely related to participating in team sports, eating regular meals, and consuming healthful foods and nutrients. The result of our study showed a non-significant relationship between BMI and tobacco use $(r=0.05, p=$ 0.65). Our findings also showed a negative, significant relationship between number of days participants engaged in at least moderate physical activity per week and tobacco use $(\mathrm{r}=-0.25, \mathrm{p}=0.05)$. This finding is congruent with the study conducted by Harrell, Speilman, and Smith (1988) on cardiovascular risk factors and socioeconomic status in 1,945 African American and Caucasian women aged 23 to 53 years. They reported that $38.8 \%$ of the African American women were obese, 51.7\% were inactive, and $31.2 \%$ smoked. After controlling for income and education, African American women were more than twice as likely as Caucasian women to be obese and to be inactive but were only half as likely to smoke.

\section{Correlations between BMI, Physical Activity, and Family History of CVD}

Family history of CVD, though a non-modifiable risk factor for cardiovascular disease, often interacts with obesity and physical inactivity to worsen the chances for the development of cardiovascular disease. Risk factors tend to occur in families and are said to be especially evident in children when an adult relative is obese. The literature noted that weight gain occurs differently in men and women. Weight gain in men occurs mostly later in life while the greatest weight gain in women occurs in the younger age groups (Krauss, Winston, Fletcher, \& Grundy, 1998). Though genetic factors play a role in the etiology of obesity and even CVD, this was not the subject of our study and was not explored. However, our findings showed no association between family history of CVD and BMI $(\mathrm{r}=-0.01 ; \mathrm{p}=0.93)$ and a non significant relationship between family history of CVD and number days of moderate physical inactivity engaged in by our study participants $(r=0.18 ; \mathrm{p}=0.16)$. 


\section{Relationships between BMI, Physical Activity, and Inflammation}

C-reactive protein (CRP) is one of the acute-phase proteins in inflammation. It has been suggested that hs-CRP is an independent marker of the risk of cardiovascular disease, though its predictive capacity remains controversial. The results from our study showed a positive significant association between BMI and hs-CRP ( $\mathrm{r}=0.51, \mathrm{p}=$ 0.0002). There was a weak positive though non-significant relationship between the number of days participants engaged in moderate physical activity and hs-CRP $(\mathrm{r}=0.12$, $p=0.41)$. These findings suggest that participants with higher BMI and those who did not meet the recommended amounts of daily physical activity had abnormal levels of hsCRP. This finding is supported by previous research studies. Rawson et al. (2003) conducted a longitudinal study examining the effects of BMI on both current and previous-year physical activity on hs-CRP in 109 healthy men and women. They reported that average BMI was correlated with current physical activity $(\mathrm{r}=0.50, \mathrm{p}<0.001)$ but was not related to previous-year physical activity levels which was assessed using the Baecke questionnaire at baseline $(r=0.02, p=0.89)$. Their data indicated that BMI but not previous-year or current physical activity predicts hs-CRP. Previous studies have also suggested an inverse relationship between physical activity and markers of inflammation such as hs-CRP. Majka et al. (2009) examined a cross-sectional association between physical activity and hs-CRP in White, Chinese, Black, and Hispanic participants enrolled in the Multi-Ethnic Study of Atherosclerosis in 2000-2002. They reported that hs-CRP was higher in women than men with black women having the highest hs-CRP level while Chinese women had the lowest even after adjusting for BMI, smoking, LDL$c$, HDL-c, blood pressure, diabetes and the use of statin, antihypertensive and diabetes medications $(\mathrm{p}=0.044)$.

\section{Correlations between Obesity, Physical Activity, and Thrombotic and Endothelial Dysfunction Markers}

According to prospective epidemiological studies, plasma fibrinogen is a newly emerging risk factor for cardiovascular disease. The deleterious effects of this protein seem to be mediated through its role in hemostasis, and the atherogenic process itself, particularly at high levels. Findings from our current study showed a positive significant relationship between BMI and plasma fibrinogen levels $(r=0.49, p=0.0004)$ and a nonsignificant association between the number of days participants engaged in daily moderate physical activity per week and plasma fibrinogen levels $(r=0.06, p=0.67)$. Mora, Lee, Buring, and Ridker (2006) studied the association of physical activity and BMI alone with hs-CRP, fibrinogen, soluble intercellular adhesion molecule-1 (sICAM1), homocystein, low-and high-density lipoprotein (LDL and HDL) cholesterol, total cholesterol, apolipoprotein A-1 and B-100, lipoprotein(a), and creatinine. The results of their study indicated that lower levels of physical activity and higher levels of BMI were independently associated ( $\mathrm{p}$ for trend $<.001$ ), with adverse levels of nearly all lipid and inflammatory biomarkers.

Soluble intercellular adhesion molecule-1 (sICAM-1) is a transmembrane protein involved in the migration and adhesion of leukocytes to the vascular endothelium. 
Available knowledge suggests that elevated baseline sICAM-1 levels predict cardiovascular disease. The findings from our study showed a positive significant association between BMI and sICAM-1 $(r=0.33, p=0.02)$. Similarly, we noted a positive significant relationship between the number of days participants engaged in moderate physical activity per week and sICAM-1 $(\mathrm{r}=0.31, \mathrm{p}=0.03)$. In a prospective evaluation of 23,984 apparently healthy women, Albert et al. (2008) found sICAM-1 levels were predictive of cardiovascular events that reflect coronary atherosclerotic disease progression and vessel narrowing $(\mathrm{p}$ trend $=0.008)$.

Plasminogen activator inhibitor 1 is the primary physiological inhibitor of plasminogen activation in vivo, and elevations in plasma PAI-1 appear to compromise normal fibrin clearance mechanisms and promote thrombosis. Findings from our study showed a non-significant positive association between BMI and PAI-1 ( $\mathrm{r}=0.21, \mathrm{p}=$ 0.15 ) as well as a non-significant relationship between the number of days participants engaged in moderate physical activity per week and PAI-1. These results are consistent with findings reported by Solano, Perry, Wang, Ross, and Goldberg (2003), suggesting that relationships reported in white populations differs in African-Americans. Solano et al., (2003), in a cross-sectional study of overweight and obese premenopausal African American and Caucasian women aged 19 to 53 years, examined the association of PAI-1 with visceral adiposity and other components of the metabolic syndrome. They found no significant association between PAI-1 and any of these variables (BMI, visceral adipose tissue, glucose, systolic blood pressure, diastolic blood pressure, triglyceride, HDL cholesterol) in African American women, despite their findings of a strong association between these metabolic variables and PAI-1 in Caucasian women. They concluded that the lack of a metabolic relationship between visceral fat and PAI-1 extends in parallel fashion to other components of the metabolic syndrome in pre-menopausal African American women. With regards to insulin resistance, they also noted there were no significant differences in PAI-1, insulin, glucose and HOMA IR values between the two groups (Solano et al., 2003). Elevated concentrations of hemostatic variables such as fibrinogen and PAI-1 have been found in both African American and Caucasian women overweight/obese women (Perry, Wang, Goldberg, Ross, \& Jackson, 2008).

Plasma E-selectin is expressed on activated endothelium. The pathogenesis of atherosclerosis involve change to the vascular endothelium which produces numerous substances involved in the regulation and maintenance of vascular integrity and the homeostasis of the coagulation/fibrinolysis system. In this study of young AfricanAmerican women, a weak positive non-significant relationship between BMI and Eselectin $(\mathrm{r}=0.21, \mathrm{p}=0.15)$ as well as a weak positive non-significant association between the number of days participant spent in moderate physical activity per week and E-selectin $(r=0.22, p=0.13)$ was noted. Limited research exists on most of these emerging cardiac risk factors. However, a review of available studies indicates sparse information regarding the association between E-selectin, BMI, and physical activity. Demerath, Towne, Blangero, and Siervogel (2001) examined cross-sectional relationships between the concentrations of soluble adhesion molecules (sICAM-1, sVCAM-1, pSelectin, and E-selectin) and smoking behavior, body composition, blood pressure, serum lipids and physical activity in 592 healthy white men and women aged 18 to 82 years. They found that men had higher E-selectin than women $(p<0.0001)$. They 
concluded that the concentration of soluble CAMs, particularly sICAM-1 and E-selectin, reflect the level of established CVD risk factors in apparently healthy men and women.

In summary, our findings are consistent with previous studies by Mora, Lee, Buring, and Ridker (2006) and Solano et al. (2003), which found an association between BMI, physical activity levels, and insulin resistance and fibrinogen levels. The differences between our findings and studies that examined relationships between the concentrations of endothelial dysfunction markers may be attributed to differences in methodology and their use of a more diverse population with a broader spectrum of weight categories.

\section{Differences in Cardiovascular Risk Factors by Weight Groups}

The third, and last, study aim compared traditional and emerging cardiovascular risk factors among normal weight and obese African American women. Obesity contributes to numerous and varied comorbid conditions. The increased incidence of obesity has also contributed to significant increases in the prevalence of most, if not all, cardiovascular disease risk factors including hypertension, dyslipidemia, insulin resistance, and type 2 diabetes. Several studies (Diez-Roux, 1999; Flegal et al., 2002) have reported that African Americans who are obese have a greater chance of having high blood pressure, diabetes, and high blood levels of triglycerides and cholesterol than blacks who are not obese and these conditions increase the risk of developing cardiovascular disease. This section will examine the differences in cardiovascular risk factors between obese and normal weight African American women.

Regardless of weight, young African American women who participated in the study had a high prevalence of traditional and emerging cardiovascular disease risk factors. Obese participants exhibited a clustering of both the traditional and emerging cardiovascular risk factors with individual obese participants having an average of 6.9 risk factors compared to 4.1 in the normal weight group. The obese group also tended to have mean values toward the cutpoints supporting the negative effect of weight on these other cardiovascular risk factors (Table 4.7). Statistically significant differences in the frequency of cardiovascular risk factors between the two weight groups were noted in only three of the risk factors: HDL-cholesterol $(p=0.01)$, hs-CRP $(p=0.00)$, and fibrinogen $(p=0.00)$, although the prevalence rates for the other cardiovascular disease risk factors was higher or similar between weight groups. Body weight or BMI did not seem to make a difference in terms of the lipid profile.

Several previous studies have examined the association of cardiovascular risk factors to body weight. The Coronary Artery Risk Development in Young Adults (CARDIA) and the Atherosclerosis Risk in Communities (ARIC) studies investigated the relationship between obesity and cardiovascular disease in African Americans (Folsom et al., 1991). The CARDIA study examined the association between behavioral and demographic factors and body size in over 2800 African American and white adults who were between the ages of 18 and 30 (Burke et al., 1992), while the ARIC study included 15,803 African Americans and white adults aged 45 to 64. In these studies, body weight, 
height, waist size, blood pressure, and blood sugar levels, and cholesterol were measured. The authors found that weight gain was more common among black women than white women and among black men than white men. Obesity in blacks was associated with higher than normal blood pressure and higher blood glucose and cholesterol levels. Diabetes was more common among blacks who were obese than among those who were thin. The lower prevalence of hypertension and diabetes seen in the women who participated in the current study may be partially attributed to their younger age. While not statistically significant, only the obese women in the current study had hypertension and diabetes. The results show that both traditional and emerging cardiovascular disease risk factors were more prevalent among the obese than normal weight subjects. This difference was statistically significant with regards to HDL-cholesterol $(p=0.02)$, hsCRP $(p=0.01)$, and fibrinogen $(p=0.002)$. These findings are similar to the results of the Multi-Ethnic Study of Atherosclerosis, which examined the relation of obesity in older men and women from diverse ethnic identities - White, African American, Hispanic, and Asian aged 45 to 84 years old (Bild et al., 2005). However, their study looked at only traditional risk factors. There are other studies that have similarly shown a higher prevalence of emerging cardiovascular risk factors among obese young adults (Appel et al., 2005; Santos et al., 2005).

In studies examining children and young adults ages 5 to 24 years, Gustat, Srinivasan, Elkasabany, and Berenson (2002), of the Bogalusa Heart Study, examined the relation of obesity to cardiovascular risk factors. They found a high prevalence of elevated systolic blood pressure, fasting insulin, and LDL-cholesterol, and low HDLcholesterol among the obese children and young adults. Although this age category differs somewhat from our study population, the findings underscore the point that obesity adversely affects cardiovascular disease risk factors throughout the lifespan.

Similarly, Nelson et al. (2002) examined a sample of low-income African American and white women $\geq 50$ years old to assess whether obesity occurs concomitantly with several coronary heart disease risk factors (diabetes, hypertension, hypercholesterolemia, low high-density lipoprotein cholesterol (HDL-C), and estimated the 10-year risk for coronary heart disease in the North Carolina WISEWOMAN (Well Integrated Screening and Evaluation for Women Across the Nation) Study sample. The results of their study showed that African American women had significantly higher BMIs, systolic blood pressure, diastolic blood pressure, and HDL-C levels than white women. The prevalence of self-reported diabetes was also significantly greater for African American women. They further reported that obese African American women were more likely to have diabetes and high blood pressure than the non-obese African American women. In contrast, our sample of obese African American women had a low prevalence of diabetes and hypertension which was similar to that found in the non-obese group. Cowie et al. (2006) reported the prevalence rates of self-reported diabetes in a nationally representative sample of African American women ages 20 to 39 and 40 to 59 were $4.7 \%$ and $12.7 \%$, respectively. Given that the mean age of participants in the current study was 31 , the overall prevalence rate of $4.7 \%$ is consistent with findings from the National Health and Nutrition Survey. 
However, impaired fasting glucose was presented in $10.4 \%$ of obese participants and $6.3 \%$ of normal weight individuals. It is recognized that type 2 diabetes is characterized by insulin resistance. Initially, hyperinsulinemia occurs in an attempt to maintain serum glucose levels. Over time, these effects are insufficient to maintain normoglycemia and prediabetes develops, presenting as either elevated fasting glucose and/or elevated post-parandial glucose levels. Further deterioration in insulin sensitivity or beta-cell function results with progression to diabetes mellitus. The literature suggests that impaired fasting glucose (prediabetes) also increases risk for cardiovascular disease. Cowie et al. (2006) reported prevalence rates of prediabetes based on fasting blood glucose levels in adults participating in the NHANES study. African American women ages $20-39$ and $40-59$ of $8.4 \%$ and $15.9 \%$ respectively which is consistent with the $16.7 \%$ overall prevalence rate of our sample. The mean age of the current study sample was 31 .

All the participants in our study had insulin resistance irrespective of their weight category. This finding is inconsistent with what has been reported in the literature. The difference may be as a result of a different protocol for measuring insulin resistance or the use of cutpoints that have been used to predict prediabetes in obese adolescents. The association between adiposity and insulin resistance has been reported in children and adults. Weight loss was associated with a decrease in insulin concentration and an increase in insulin sensitivity (Arslanian, Suprasongsin, \& Janosky, 1997). African Americans have been reported to have the greater prevalence of insulin resistance, hypertension, diabetes and obesity and these may contribute to vascular disease (Falkner, Hulman, Tannenbaum, \& Kushner, 1990).

Gustat et al. (2002) examined the relation of self-rated measures of physical activity to multiple risk factors of insulin resistance syndrome (IRS) in African American and white young adults aged 20 to 38 years enrolled in the Bogalusa Heart Study. They found an inverse relationship between leisure-time activity and mean number of risk factors whereas a positive relationship was seen with hours of inactivity $(p<0.001)$. Moderate to very active individuals versus inactive individuals had a reduced relative risk of having $>$ or $=3$ risk factors determined by waist circumference, blood pressure, total to high-density lipoprotein cholesterol ratio, and insulin resistance index showed a positive trend with hours of inactivity $(\mathrm{p}<0.01)$. Thus, the low levels of physical activity reported by individuals, with the majority of their activity being moderate or less intense, may contribute to the insulin resistance found in this sample of young African Americans.

Data from the 1999-2004 National Health and Nutrition Examination Survey examined whether obesity's association with novel cardiovascular risk factors such as Creactive protein varies by race. Wee et al. (2008) noted an association between obesity and CRP in African Americans. Research investigating the role of obesity in inducing upregulation of adhesion molecules such as SICAM-1, VCAM-1, and E-selectin levels in 40 obese and 30 non-obese patients with type 2 diabetes (Matsumoto et al., 2002) suggested that sICAM-1 and VCAM-1 levels did not differ significantly between obese and non-obese patients. However, serum concentrations of E-selectin levels were significantly higher in obese than in non-obese patients. This report was further supported by Nijhuis et al. (2007) in their investigation of the role of obesity in endothelial cell activation following long term weight loss after bariatric surgery. They noted that despite 
substantial weight loss during the first six months post-bariatric surgery, E-selectin levels decreased while sICAM-1 and sVCAM-1 plasma levels did not decrease significantly. These reports differ from our study finding partly because there is no defined normal reference for these novel risk markers yet.

Increased levels of risk factors such as PAI-1 and fibrinogen have also been associated with increased risk for cardiovascular disease. The literature is equally clear on the relationship between plasma PAI-1 and obesity as well as between fibrinolysis and obesity. Studies performed in obese and normal weight subjects suggested that obese subjects had higher plasma concentrations of PAI-1 as compared to non-obese controls (Hong, Pedersen, Egberg, \& de Faire, 1997). Similar studies show that obesity may account for higher concentrations of fibrinogen with plasma levels of fibrinogen correlating with central obesity independently of metabolic factors (Mertens \& Van Gaal, 2002).

\section{Strengths and Limitations}

The strength of this study is rooted in its uniqueness in that it sought to provide much needed information on the association between obesity and cardiovascular disease risk factors in African American women. Data on this population have been sparse despite African Americans having the highest rate of cardiovascular morbidity and mortality. The study not only expands knowledge about this understudied population but also provides direction for policy, clinical practice, and future research. The focus of the study on African American women aged 18 to 45 helps to illustrate the potential benefits of early screening in a population that would otherwise be considered low-risk. It has been recognized that though symptomatic cardiovascular disease is less common in premenopausal women, the process of atherosclerosis starts long before menopause and earlier intervention might be useful in reducing the long-term morbidity and mortality associated with cardiovascular disease.

This study is not without limitations. The cross-sectional descriptive correlational design allows researchers to gather data from one point in time. This does not reflect the totality of the participants' experiences hence the findings, though valid, may not necessarily represent the influence of body weight on cardiovascular disease risk factors over time. A longitudinal research study might be a better tool in assessing the long-term relationship between body weight and cardiovascular disease risk factors. Another inherent limitation is the small sample size and lack of regional and cultural diversity. All the participants were recruited from two adjoining states from the mid-south, and the findings may not be characteristic of other African American women from other parts of the country. The use of self-reporting instruments to elicit information regarding illicit drug use, smoking history, and even physical activity levels is another limitation of the study due to potential recall bias and under reporting. The emerging cardiovascular disease risk factors are by definition novel without established cut-points which raises questions on the validity of cut-points used in this study. 


\section{Relation of Study Results to Conceptual Framework}

This study examined the relationship between obesity and the traditional and emerging cardiovascular risk factors. Cardiac risk factors are good indicators of an individual's overall risk of developing heart disease. The conceptual model (see Figure 1.1) depicts major risk factors for cardiovascular disease and their interrelationship. Obesity is not just one of the traditional modifiable risk factors for cardiovascular disease but is also associated with increased prevalence of other risk factors such as diabetes, hypertension, and dyslipidemia. The findings of our study show a higher prevalence of physical inactivity, tobacco use, dyslipidemia, and diabetes among obese participants with statistical significance noted in only HDL-cholesterol levels.

While our study had controlled for age, gender, and ethnicity or race (all nonmodifiable traditional cardiovascular risk factors), it is, however, a known fact that higher rates of coronary heart disease have been reported among men compared with women with increased risk of stroke among African Americans. It is equally well established that advancing age is an independent risk factor for cardiovascular disease as the risk of stroke is said to double every decade after age 55 . The result of our study shows no statistical difference between the obese and normal weight participants who reported a family history of cardiovascular disease. These non-modifiable risk factors have been known to affect or influence development of the modifiable risk factors.

Emerging risk factors such as fibrinogen, triglycerides, insulin resistance, hsCRP, PAI-1, sICAM-1, and E-selectin are novel risk factors that occur in obese people either correlate with traditional cardiovascular risk factors or predict subsequent cardiovascular events. Our findings showed that these risk factors were elevated in obese participants with statistical significance noted in hs-CRP and fibrinogen levels.

Cardiovascular risk and outcome are affected by socio-cultural factors. More of the participants in the obesity group reported having high school education or lower and earned less annual household income compared to the normal weight group. Even though we did not evaluate the health belief systems or their health seeking behaviors, we ascertained that all the study participants had health insurance coverage. Griffith, Moy, Reischl, and Dayton (2006) described five models that explain disparities in cardiovascular disease outcomes in African American women. Racial-genetic, healthbehavior, socioeconomic, psychosocial stress, and structural-constructivist models were identified as possible explanations for the observed disparities in cardiovascular disease burden and outcome among different racial and ethnic groups. It is a known fact that inherent biological differences among ethnic groups are the determinants of disease burden and outcomes. This suggests that there are differences between racial and ethnic groups in the distribution of individual health behaviors such as physical activity and tobacco use. Cultural differences are thought to explain disparities in health outcome with socioeconomic status believed to be a primary determinant of health and disease while psychosocial stress model attributes health disparities to the discrimination and racism experienced by ethnic minorities who often suffer as a result of the power structure and centuries of exploitation, discrimination and racism. This explains the association 
between socio-cultural factors and the emerging risk factors as well as the traditional non-modifiable cardiac risk factors.

\section{Practice and Policy Implications}

These findings carry implications for clinical practice: obese adults without clinical cardiovascular disease may already have a clustering of both traditional and emerging cardiovascular risk factors. Institution of early and aggressive preventive measures in this population could delay or arrest progression to clinical cardiovascular disease.

The knowledge gained by conducting this descriptive correlation analysis could provide health care providers with the tools they need to educate and inform women about their risks for cardiovascular disease and help them develop strategies for disease prevention. To reduce the high morbidity and mortality of cardiovascular disease among African American women, regular cardiovascular disease screening and intervention should be started in early adulthood. Contrary to prevailing beliefs that heart disease is rare among women and highly unlikely in premenopausal women, health care providers should be reeducated on the need of cardiovascular risk assessment in young adults.

A collaborative effort between health care professionals and members of minority communities is necessary to provide effective outcomes that increase physical activity in this population. African American women like other people should be encouraged to seek regular medical evaluation with detailed screening for risk factors even when asymptomatic as this may represent the best opportunity to limit the devastating effects of cardiovascular disease. They should also be encouraged to be proactive in advocating for their health. City and state governments should embark on a variety of educational and public awareness activities to educate the citizenry particularly ethnic minorities about their cardiovascular risk factors by helping them to learn more about their risk for heart disease so they can take action to lower the risk.

The indisputable benefit of early screening and primary prevention should compel insurance payers to compensate clinicians who provide such care. In line with several study findings (Chopra, Choksi, \& Cavusoglu, 2007; Ridker et al., 2008), it could be beneficial to treat asymptomatic individuals some of whom may have prehypertension or impaired glucose tolerance with several anti-inflammatory medications (such as statins) that are currently reserved for those already determined to have disease. The benefits of this should be carefully weighed against the risks in these women of child bearing age.

\section{Recommendations for Future Research}

The findings of this descriptive correlational study provide various opportunities for future research into the cardiovascular health of African American women. A longitudinal study that examines the association between obesity and the traditional and

emerging cardiovascular disease risk factors is highly recommended. Such a study would 
provide more insight into the complex mechanisms at work in cardiovascular disease, particularly with risk stratification in this vulnerable population. Also a longitudinal study of these women to determine their outcomes overtime and if and how these risk factors change to examine the natural history of these risk markers would be of additional benefit. Besides conducting a longitudinal study, it would be equally beneficial to sample a larger population of African American women across different regions of the United States for a more representative sample. Such a diverse population would make generalization of the study sample feasible. Another suggestion for future research is to examine obese persons who engage in recommended amounts of physical activity and those who do not to determine if there is a difference in the levels of the risk factors between the groups. This is because there is a growing thought that obese persons who are physically fit are less likely to manifest the risk factors for cardiovascular disease. Although an examination of possible barriers to cardiovascular health was beyond the scope of this study, it is recommended for further research of this population. Finally, it would make much sense to conduct similar research on women of other ethnicities to determine if differences exist in the manifestation of these risk factors for heart disease.

\section{Conclusions}

Obesity is highly prevalent among African American women. We found that the coronary heart disease risk factors that often occur with obesity clustered in the African American women, whether they were obese or not, whereas more risk factors occurred in the presence of obesity. Among African American women there was no statistically significant difference for the clustering of triglycerides, LDL-cholesterol, total cholesterol, physical inactivity, smoking, hypertension, PAI-1, E-selectin, and sICAM-1 between obese and non-obese women. This knowledge is relevant given the fact that literature is scarce regarding the prevalence of the emerging cardiovascular risk factors in African American women. Moreover, previous studies have reported a relationship between obesity and cardiovascular disease risk factors among ethnic minorities.

The result of our study is alarming, given the increased national awareness of cardiovascular risk among the United States general population. The continued lifestyle that excludes regular physical activity and the prevalence of both the traditional and emerging risk factors for heart disease among our study population is a public health concern. Targeted media campaigns and intervention programs should be directed to this at-risk population in order to promote healthy lifestyle change within their financial and time constraints. Health care providers should engage in widespread cardiovascular disease prevention education programs that are culturally relevant to this underserved population. Governmental intervention is needed to increase access to care, support health promotion information, and provide resources to aid low-income African American women who may lack awareness of the importance of cardiovascular disease risk prevention and management. Given the advancement in technology, medical breakthroughs, and increased scientific knowledge, one should hope that cardiovascular disease will no longer be the leading cause of death among women in the United States. 


\section{LIST OF REFERENCES}

Association of American Medical Colleges. (2008). Heart disease in women-Getting the attention it deserves. Retrieved September 1, 2008, from

http://www.aamc.org/research/ftp/ftpheartdisease.pdf

Abdu, T., Elhadd, T., Pfeifer, M., \& Clayton, R. (2001). Endothelial dysfunction in endocrine disease. Trends in Endocrinology \& Metabolism, 12(6), 257-265.

Adeniyi, A., Folsom, A. R., Brancati, F. L., Desvorieux, M., Pankow, J. S., \& Taylor, H. (2002). Incidence and risk factors for cardiovascular disease in African Americans with diabetes: The Atherosclerosis Risk in Communities (ARIC) study. Journal of National Medical Association, 94(12), 1025-1035.

Ainsworth, B. E., Haskell, W. L., Whitt, M. C., Irwin, M. L., Swartz, A. M., Strath, S. J., et al. (2000). Compendium of physical activities: An update of activity codes and MET intensities. Medicine \& Science in Sports \& Exercise, 32 (9 Suppl), S498-504.

Albert, M., Glynn, R., Buring, J., \& Ridker, P. (2006). Impact of traditional and novel risk factors on the relationship between socioeconomic status and incident cardiovascular events. Circulation, 114(24), 2619.

Albert, M., \& Ridker, P. (2006). C-reactive protein as a risk predictor: Do race/ethnicity and gender make a difference? Circulation, 114(5), e67.

Alberti, K. G., \& Zimmet, P. Z. (1998). Definition, diagnosis, and classification of diabetes and its complications. Part 1: Diagnosis and classification of diabetes mellitus. Provisional report of a WHO consultation. Diabetic Medicine, 15(7): 539-553.

Allain, C. C., Poon, L. S., Chan, C. S., Richmond, W., \& Fu, P. C. (1974). Enzymatic determination of total serum cholesterol. Clinical Chemistry, 20(4), 470-475.

American Council on Exercise. (2001). Fit facts: Monitoring exercise intensity using heart rate. Retrieved August 22, 2008, from

http://www.acefitness.org/fitfacts/pdfs/fitfacts/itemid_38.pdf

American Diabetes Association. (2007). Clinical practice recommendations. Retrieved February 15, 2009, from http://www.diabetes.org/for-health-professionals-and -scientists/cpr.jsp

American Diabetes Association. (2008). Diagnosis and classification of diabetes mellitus. Diabetes Care, 31(1 Suppl), S55-60.

American Heart Association. (2009d). Risk factors and coronary heart disease. Retrieved January 5, 2009, from http://www.americanheart.org/presenter.jhtml?identifier=4726 
American Heart Association. (2006). Heart disease and stroke statistics-2006 update. Dallas, Texas: American Heart Association; 2005.

American Heart Association. (2008). Heart disease and stroke statistics. Retrieved November 30, 2008, from

http://www.americanheart.org/download/heart/1200082005246HS Stats\%202008.final. $\underline{\mathrm{pdf}}$

Amercan Heart Association. (2009a). AHA statistical update: heart disease and stroke statistics---2009 update: A report from the American Heart Assocation Statistics

Committee and Stroke Statistics Subcommittee. Circulation. Retrieved January 31, 2009, from http://circ.ahajournals.org/cgi/reprint/CIRCULATIONAHA.108.191261

American Heart Association. (2009b). Inflammation, heart disease and stroke: The role of C-reactive protein. Retrieved March 9, 2009, from

http://www.americanheart.org/presenter.jhtml?identifier $=4648$

American Heart Association. (2009c). Obesity and overweight. Retrieved January 15, 2009, from http://www.americanheart.org/presenter.jhtml?identifier=4639

American Obesity Association (2002). AOA Fact Sheets: Women and obesity. Retrieved July 4, 2008, from

http://obesity1.tempdomainname.com/subs/fastfacts/obesity_women.shtml

Appel, S. J., Harrell, J. S., \& Davenport, M. L. (2005). Central obesity, the metabolic syndrome, and plasminogen activator inhibitor-1 in young adults. Journal of American Academy of Nurse Practitioners, 17(12), 535-541.

Arslanian, S. A., Suprasongsin, C., \& Janosky, J. E. (1997). Insulin secretion and sensitivity in black versus white prepubertal healthy children. Journal of Clinical Endocrinology \& Metabolism, 82(6), 1923-1927.

Ayanian, J., Landon, B., Landrum, M., Grana, J., \& McNeil, B. (2002). Use of cholesterol-lowering therapy and related beliefs among middle-aged adults after myocardial infarction. Journal of General Internal Medicine, 17(2), 95-102.

Bakris, G., Hill, M., Mancia, G., Steyn, K., Black, H., Pickering, T., et al. (2007). Achieving blood pressure goals globally: Five core actions for health-care professionals. A worldwide call to action. Journal of Human Hypertension, 22(1), 63.

Bassett, D. R., Jr., Fitzhugh, E. C., Crespo, C. J., King, G. A., \& McLaughlin, J. E. (2002). Physical activity and ethnic differences in hypertension prevalence in the United States. Preventive Medicine, 34(2), 179-186. 
Bertuzzi, M., Negri, E., Tavani, A., \& La Vecchia, C. (2003). Family history of ischemic heart disease and risk of acute myocardial infarction. Preventive Medicine, 37(3), 183187.

Bild, D. E., Detrano, R., Peterson, D., Guerci, A., Liu, K., Shahar, E. et al. (2005). Ethnic differences in coronary calcification: The Multi-Ethnic Study of Atherosclerosis (MESA). Circulation, 111, 1313-1320.

Blair, S. N. (1996). Physical inactivity and cardiovascular disease risk in women. Medicine \& Science in Sports \& Exercise, 28(1), 9-10.

Blair, S. N., Applegate, W. B., Dunn, A. L., Ettinger, W. H., Haskell, W. L., King, A. C., et al. (1998). Activity Counseling Trial (ACT): Rationale, design, and methods. Activity Counseling Trial Research Group. Medicine \& Science in Sports \& Exercise, 30(7), 1097-1106.

Blair, S. N., \& Brodney, S. (1999). Effects of physical inactivity and obesity on morbidity and mortality: current evidence and research issues. Medicine \& Science in Sports \& Exercise, 31(11 Suppl), S646-662.

Blustein, J. \& Weitzman, B. C. (1995). Access to hospitals with high-technology cardiac services: How is race important? American Journal of Public Health, 85(3), pp. 345-351.

Bonora, E. (2006). The metabolic syndrome and cardiovascular disease. Annals of Medicine, 38(1), 64-80.

Brownson, R., Smith, C., Jorge, N., Dean, C., \& DePrima, L. (1992). Controlling cardiovascular disease: The role of the local health department. American Journal of Public Health, 82(10), 1414-1416.

Burke, G. L., Savage, P. J., Manolio, T. A., Sprafka, J. M., Wagenknecht, L. E., Sidney, S. et al. (1992). Correlates of obesity in young black and white women: The CARDIA study. American Journal of Public Health, 82(12), 1621-1625.

Burkhauser, R., \& Cawley, J. (2008). Beyond BMI: The value of more accurate measures of fatness and obesity in social science research. Journal of Health Economics, 27(2), 519-529.

Burns, N. \& Grove, S. K. (Ed.). (1993). The practice of nursing research: Conduct, critique, and utilization (2nd ed.). Philadelphia: W. B. Saunders.

Carty, C., Cushman, M., Jones, D., Lange, L., Hindorff, L., Rice, K., et al. (2008). Associations between common fibrinogen gene polymorphisms and cardiovascular disease in older adults. The Cardiovascular Health Study. Thrombosis \& Haemostasis, 99(2), 388-395. 
Caspersen, C. J., Powell, K. E., \& Christenson, G. M. (1985). Physical activity, exercise, and physical fitness: Definitions and distinctions for health-related research. Public Health Report, 100(2), 126-131.

Cassano, P., Rosner, B., Vokonas, P., \& Weiss, S. (1992). Obesity and body fat distribution in relation to the incidence of non-insulin-dependent diabetes mellitus: A prospective cohort study of men in the normative aging study. American Journal of Epidemiology, 136(12), 1474-1486.

Castelli, W. (1984). Epidemiology of coronary heart disease: The Framingham Study. American Journal of Medicine, 76(2A), 4-12.

Centers for Disease Control \& Prevention. (1999). Physical activity and health: A report of the Surgeon General executive summary. Retrieved January 4, 2009, from http://www.cdc.gov/nccdphp/sgr/pdf/execsumm.pdf

Centers for Disease Control \& Prevention. (2007a). Physical activity and health. U.S. Department of Health and Human Services. Retrieved August 8, 2008, from http://www.cdc.gov/nccdphp/sgr/women.htm

Centers for Disease Control \& Prevention. (2007b). A report of the Surgeon General: Physical activity and health-Adults. Retrieved August 19, 2008, from http://www.fitness.gov/adults.pdf

Centers for Disease Control \& Prevention. (2008). U.S. Physical activity statistics. Retrieved August 19, 2008, from http://apps.nccd.cdc.gov/PASurveillance/StateSumV.asp

Centers for Disease Control \& Prevention and National Center for Health Statistics (2006). Obesity still a major problem. Retrieved February 16, 2009, from http://www.cdc.gov/nchs/pressroom/06facts/obesity03 04.htm

Cefalu, W. (2001). Insulin resistance: Cellular and clinical concepts. Experimental Biology \& Medicine, 226, 13-26.

Chandrashekhar, Y., \& Anand, I. S. (1991). Exercise as a coronary protective factor. American Heart Journal, 122(6), 1723-1739.

Chikani, V., Reding, D., Gunderson, P., \& McCarty, C. (2004). Wisconsin Rural Women's Health Study on psychological factors and blood cholesterol level: Difference between normal and overweight rural women. Clinical Medicine \& Research, 2(1), 4753. 
Chobanian, A. V., Bakris, G L., Black, H. R., Cushman, W. C., Green, L. A., Izzo, J. L. et al. (2003). The seventh report of the Joint National Committee on Prevention, Detection, Evaluation, and Treatment of High Blood Pressure: The JNC 7 report. JAMA, 289(19), 2560-2571.

Chopra, V., Choksi, P. U., \& Cavusoglu, E. (2007). Beyond lipid lowering: The antihypertensive role of statins. Cardiovascular Drugs and Therapy, 21(3), 161-169.

Cleeman, J., \& Lenfant, C. (1998). The National Cholesterol Education Program progress and prospects. $J A M A, 280(24), 2099-2104$.

Cleland, S., Petrie, J., Ueda, S., Elliott, H., \& Connell, J. (1998). Insulin as a vascular hormone: Implications for the pathophysiology of cardiovascular disease. Clinical and Experimental Pharmacology and Physiology, 25(3-4), 175-184.

Cossrow, N., \& Faulkner, B. (2004). Race/ethnic issues in obesity and obesity-related comorbidities. Journal of Clinical Endocrinology \& Metabolism, 89(6), 2590-2594.

Cowie, C. C., Rust, K. F., Byrd-Holt, D. D., Eberhardt, M. S., Flegal, K. M., Engelgau, M. M. et al. (2006). Prevalence of diabetes and impaired fasting glucose in adults in the U.S. population: National Health and Nutrition Examination Survey 1999-2002. Diabetes Care, 29, 1263-1268.

D'Agostino, R. B., Sr., Grundy, S., Sullivan, L. M., \& Wilson, P. (2001). Validation of the Framingham coronary heart disease prediction scores: Results of a multiple ethnic groups investigation. JAMA, 286(2), 180-187.

Dalton, M., Cameron, A. J., Zimmet, P. Z., Shaw, J. E., Jolley, D., Dunstan, D. W., et al. (2003). Waist circumference, waist-hip ratio and body mass index and their correlation with cardiovascular disease risk factors in Australian adults. Journal of Internal Medicine, 254(6), 555-563.

Danesh, J., Whincup, P., Walker, M., Lennon, L., Thomson, A., Appleby, P., et al. (2000). Low grade inflammation and coronary heart disease: Prospective study and updated meta-analyses. British Medical Journal, 321(7255), 199-204.

Dawber, T., Meadors, G., \& Moore Jr., F. (1951). Epidemiological approaches to heart disease: The Framingham Study. American Journal of Public Health, 41(3), 279-281.

De, S., Searles, G., \& Haddad, H. (2002). The prevalence of cardiac risk factors in women 45 years of age or younger undergoing angiography for evaluation of undiagnosed chest pain. Canadian Journal of Cardiology, 18(9), 945-948.

Demerath, E., Towne, B., Blangero, J., \& Siervogel, R. M. (2001). The relationship of soluble ICAM-1, VCAM-1, P-selectin and E-selectin to cardiovascular disease risk factors in healthy men and women. Annals of Humun Biology, 28(6), 664-678. 
Despres, J. P., Moorjani, S., Lupien, P. J., Tremblay, A., Nadeau, A., \& Bouchard, C. (1990). Regional distribution of body fat, plasma lipoproteins, and cardiovascular disease. Arteriosclerosis, 10(4), 497-511.

Di Napoli, M., Papa, F., \& Bocola, V. (2001). Prognostic influence of increased Creactive protein and fibrinogen levels in ischemic stroke. Stroke, 32, 133-138.

Diez-Roux, A. (1999). Prevalence and social correlates of cardiovascular disease risk factors in Harlem. American Journal of Public Health, 89(3), 302-307.

Dowling, H. J., Fried, S. K., \& Pi-Sunyer, F. X. (1995). Insulin resistance in adipocytes of obese women: Effects of body fat distribution and race. Metabolism, 44(8), 987-995.

Eriksson, K. F., \& Lindgarde, F. (1996). Poor physical fitness, and impaired early insulin response but late hyperinsulinaemia, as predictors of NIDDM in middle-aged Swedish men. Diabetologia, 39(5), 573-579.

Erkelens, D. W. (2001). Insulin resistance syndrome and type 2 diabetes mellitus. American Journal of Cardiology, 88(7B), 38J-42J.

Ernst, E. (1993). Fibrinogen as a cardiovascular risk factor-Interrelationship with infections and inflammation. European Heart Journal, 14(K Suppl), 82-87.

Falkner, B., Hulman, S., Tannenbaum, J., \& Kushner, H. (1990). Insulin resistance and blood pressure in young black men. Hypertension, 16(6), 706-711.

Felton, G. M., Boyd, M. D., Bartoces, M. G., \& Tavakoli, A. S. (2002). Physical activity in young African American women. Health Care for Women International, 23(8), 905918.

Ferdinand, K. C. (2006). Ethnic, gender, and age-related differences in the treatment of dyslipidemia. American Journal of Managed Care, 12(15 Suppl), S400-S404.

Ferdinand, K. C. (2007). African American heart failure trial: Role of endothelial dysfunction and heart failure in African Americans. American Journal of Cardiology, 99(6B), 3D-6D.

Ferns, G. A. (2008). New and emerging risk factors for CVD. Proceedings of the Nutrition Society, 67(2), 223-231.

Festa, A., D'Agostino, R., Jr., Williams, K., Karter, A. J., Mayer-Davis, E. J., Tracy, R. P., et al. (2001). The relation of body fat mass and distribution to markers of chronic inflammation. International Journal of Obesity Related Metabolic Disorders, 25(10), 1407-1415. 
Finkelstein, E., Fiebelkorn, I., \& Wang, G. (2003). National medical spending attributable to overweight and obesity: How much, and who's paying? Health Affairs, W3, 219-226.

Finkelstein, E., Fiebelkorn, I., \& Wang, G. (2004). State-level estimates of annual medical expenditures attributable to obesity. Obesity Research, 12(1), 18-24.

Flegal, K. M., Carroll, M. D., Ogden, C. L., \& Johnson, C. L. (2002). Prevalence and trends in obesity among US adults, 1999-2000. JAMA, 288(14), 1723-1727.

Folsom, A. R., Burke, G. L., Byers, C. L., Hutchinson, R. G., Heiss, G., Flack, J. M., et al. (1991). Implications of obesity for cardiovascular disease in blacks: The CARDIA and ARIC studies. American Journal of Clinical Nutrition, 53(6 Suppl), 1604S-1611S.

Ford, E., Giles, W., \& Dietz, W. (2002). Prevalence of the metabolic syndrome among US adults: Findings from the Third National Health and Nutrition Examination Survey. JAMA, 287(3), 356-359.

Ford, E. S. (1999). Body mass index, diabetes, and C-reactive protein among U.S. adults. Diabetes Care, 22(12), 1971-1977.

Freedman, D. S., Khan, L. K., Serdula, M. K., Galuska, D. A., \& Dietz, W. H. (2002). Trends and correlates of class 3 obesity in the United States from 1990 through 2000. $J A M A, 288(14), 1758-1761$.

Freestone, B., Lip, G., Chong, A., Nadar, S., Lee, K., Blann, A., et al. (2005). Circulating endothelial cells in atrial fibrillation with and without acute cardiovascular disease. Thrombosis \& Haemostasis, 94(4), 702-706.

Friedewald, W. T., Levy, R. I., \& Fredrickson, D. S. (1972). Estimation of the concentration of low-density lipoprotein cholesterol in plasma, without use of the preparative ultracentrifuge. Clinical Chemistry, 18(6), 499-502.

Fung, T. T., Rimm, E. B., Spiegelman, D., Rifai, N., Tofler, G. H., Willett, W. C., et al. (2001). Association between dietary patterns and plasma biomarkers of obesity and cardiovascular disease risk. American Journal of Clinical Nutrition, 73(1), 61-67.

Gardner, C., Winkleby, M., \& Fortmann, S. (2000). Population frequency distribution of non-high-density lipoprotein cholesterol (Third National Health and Nutrition Examination Survey, 1988-94). American Journal of Cardiology., 86, 299-304.

Gelber, R., Gaziano, J., Orav, E., Manson, J., Buring, J., \& Kurth, T. (2008). Measures of obesity and cardiovascular risk among men and women. Journal of American College of Cardiology, 52(8), 605. 
Giles-Corti, B., Timperio, A., Cutt, H., Pikora, T., Bull, F., Knuiman, M., et al. (2006). Development of a reliable measure of walking within and outside the local neighborhood: RESIDE's Neighborhood Physical Activity Questionnaire. Preventive Medicine, 42(6), 455-459.

Gils, A. (2006). The pathophysiological relevance of PAI-1 in cardiovascular diseases and the development of monoclonal antibodies as PAI-1 inhibitors. Verh K Acad Geneeskd Belg, 68(3), 179-198.

Glew, R. H., Conn, C. A., Vanderjagt, T. A., Calvin, C. D., Obadofin, M. O., Crossey, M., et al. (2004). Risk factors for cardiovascular disease and diet of urban and rural dwellers in northern Nigeria. Journal of Health, Population \& Nutrition, 22(4), 357-369.

Goff, J., DC, Labarthe, D., Howard, G., \& Russell, G. (2002). Primary prevention of high blood cholesterol concentrations in the United States. Archives of Internal Medicine, 162(8), 913-919.

Gower, B. A. (1999). Syndrome X in children: Influence of ethnicity and visceral fat. American Journal of Human Biology, 11(2), 249-257.

Griffith, D. M., Moy, E., Reischl, T. M., \& Dayton, E. (2006). National data for monitoring and evaluating racial and ethnic health inequities: Where do we go from here? Health Education \& Behavior, 33(4), 470-487.

Grundy, S. (1997). Small LDL, atherogenic dyslipidemia, and the metabolic syndrome. Circulation, 95(1), 1-4.

Grundy, S. M., Hansen, B. Smith, S. C., Jr., Cleeman, J. I., \& Kahn, R. A. (2004). Clinical management of metabolic syndrome: Report of the American Heart Association/National Heart, Lung, and Blood Institute/American Diabetes Association Conference on Scientific Issues Related to Management. Circulation, 109: 551-556.

Gurevitz, O., Jonas, M., Boyko, V., Rabinowitz, B., \& Reicher-Reiss, H. (2000). Clinical profile and long-term prognosis of women $<$ or $=50$ years of age referred for coronary angiography for evaluation of chest pain. American Journal of Cardiology, 85(7), 806809.

Gustat, J., Srinivasan, S. R., Elkasabany, A., \& Berenson, G. S. (2002). Relation of selfrated measures of physical activity to multiple risk factors of insulin resistance syndrome in young adults: the Bogalusa Heart Study. Journal of Clinical Epidemiology, 55(10), 997-1006.

Haffner, S., \& Taegtmeyer, H. (2003). Epidemic obesity and the metabolic syndrome. Circulation, 108, 1541-1545. 
Haffner, S. M., D'Agostino, R., Saad, M. F., Rewers, M., Mykkanen, L., Selby, J., et al. (1996). Increased insulin resistance and insulin secretion in nondiabetic AfricanAmericans and Hispanics compared with non-Hispanic whites. The Insulin Resistance Atherosclerosis Study. Diabetes, 45(6), 742-748.

Hajjar, I., \& Kotchen, T. A. (2003). Regional variations of blood pressure in the United States are associated with regional variations in dietary intakes: The NHANES III data. American Society for Nutritional Sciences, 133, 211-214.

Hall, W., Watkins, L., Wright Jr, J., Wenger, N., Kumanyika, S., Gavin III, J., et al. (2006). The Metabolic Syndrome: Recognition and Management. Disease Management, 9(1), 16-33.

Harrell, J. F., Speilman, D., \& Smith, A. C. (1988). Repair of a distal leg defect using the latissimus dorsi free flap. Journal of the American Osteopathic Association, 88(12), 1516-1518.

Helmrich, S. P., Ragland, D. R., Leung, R. W., \& Paffenbarger, R. S., Jr. (1991). Physical activity and reduced occurrence of non-insulin-dependent diabetes mellitus. New England Journal of Medicine, 325(3), 147-152.

Hill, J. O., Sidney, S., Lewis, C. E., Tolan, K., Scherzinger, A. L., \& Stamm, E. R. (1999). Racial differences in amounts of visceral adipose tissue in young adults: The CARDIA (Coronary Artery Risk Development in Young Adults) Study. American Journal of Clinical Nutrition, 69(3), 381-387.

Ho, S. C., Chen, Y. M., Woo, J. L., Leung, S. S., Lam, T. H., \& Janus, E. D. (2001). Association between simple anthropometric indices and cardiovascular risk factors. International Journal of Obesity Related Metabolic Disorders, 25(11), 1689-1697.

Hong, Y., Pedersen, N. L., Egberg, N., \& de Faire, U. (1997). Moderate genetic influences on plasma levels of plasminogen activator inhibitor-1 and evidence of genetic and environmental influences shared by plasminogen activator inhibitor-1, triglycerides, and body mass index. Arteriosclerosis, Thrombosis, and Vascular Biology, 17, 27762782.

Howard, B. V., Mayer-Davis, E. J., Goff, D., Zaccaro, D. J., Laws, A., Robbins, D. C., et al. (1998). Relationships between insulin resistance and lipoproteins in nondiabetic African Americans, Hispanics, and non-Hispanic whites: The Insulin Resistance Atherosclerosis Study. Metabolism, 47(10), 1174-1179.

Howes, J. M., Keen, J. N., Findlay, J. B., \& Carter, A. M. (2008). The application of proteomics technology to thrombosis research: The identification of potential therapeutic targets in cardiovascular disease. Diabetes \& Vascular Disease Research, 5(3), 205-212. 
Hu, F., Manson, J., Stampfer, M., Colditz, G., Liu, S., Solomon, C., et al. (2001). Physical activity and risk for cardiovascular events in diabetic women. Annals of Internal Medicine, 134(2), 96-105.

Jacobs, E., Rolle, I., Ferrans, C., Whitaker, E., \& Warnecke, R. (2006). Understanding African Americans' views of the trustworthiness of physicians. Journal of General Internal Medicine, 21(6), 642-647.

Janand-Delenne, B., Chagnaud, C., Raccah, D., Alessi, M., Juhan-Vague, I., \& Vague, P. (1998). Visceral fat as a main determinant of plasminogen activator inhibitor 1 level in women. International Journal of Obesity, 22, 312-317.

Jenny, N., Arnold, A., Kuller, L., Tracy, R., \& Psaty, B. (2007). Serum Amyloid P and cardiovascular disease in older men and women: Results from the Cardiovascular Health Study. Arteriosclerosis, Thrombosis, and Vascular Biology, 27(2), 352.

Jiang, X., Srinivasan, S. R., Radhakrishnamurthy, B., Dalferes, E. R., \& Berenson, G. S. (1996). Racial (black-white) differences in insulin secretion and clearance in adolescents: the Bogalusa Heart Study. Pediatrics, 97(3), 357-360.

JNC 7 (2003). The Seventh Report of the Joint National Committee on Prevention, Detection, Evaluation and Treatment of High Blood Pressure: Accurate blood pressure measurement in the office. Retrieved March 1, 2008, from http:/www.nhlbi.nih.gov/guidelines/hypertension/expresss.pdf

Johnson-Kozlow, M., Rock, C., Gilpin, E., Hollenbach, K., \& Pierce, J. (2007). Validation of the WHI Brief Physical Activity Questionnaire among women diagnosed with breast cancer. American Journal of Health Behavior, 31(2), 193-202.

Kadish, A. H., \& Hall, D. A. (1965). A new method for the continuous monitoring of blood glucose by measurement of dissolved oxygen. Clinical Chemistry, 11(9), 869-875.

Kanjilal, S., Rao, V. S., Mukherjee, M., Natesha, B. K., Renuka, K. S., Sibi, K., et al. (2008). Application of cardiovascular disease risk prediction models and the relevance of novel biomarkers to risk stratification in Asian Indians. Vascular Health \& Risk Management, 4(1), 199-211.

Katz, A., Nambi, S., Mather, K., Baron, A., Follmann, D., Sullivan, G., et al. (2000). Quantitative Insulin Sensitivity Check Index: A simple, accurate method for assessing insulin sensitivity in humans. Endocrine Society, 85, 2402-2410.

Knowler, W. C., Barrett-Connor, E., Fowler, S. E., Hamman, R. F., Lachin, J. M., Walker, E. A., et al. (2002). Reduction in the incidence of type 2 diabetes with lifestyle intervention or metformin. New England Journal of Medicine, 346(6), 393-403. 
Krauss, R. M., Winston, M., Fletcher, B. J., \& Grundy, S. M. (1998). Obesity: Impact on cardiovascular disease. Circulation, 98(14), 1472-1476.

Krishnan, S., Rosenberg, L., Djousse, L., Cupples, L. A., \& Palmer, J. R. (2007). Overall and central obesity and risk of type 2 diabetes in U.S. black women. Obesity (Silver Spring), 15(7), 1860-1866.

Krishnan, S., Rosenberg, L., \& Palmer, J. R. (2008). Physical activity and television watching in relation to risk of type 2 diabetes: The Black Women's Health Study. American Journal of Epidemiology, 163(Suppl), S313.

Kullo, I., Jan, M., Bailey, K., Mosley, T., \& Turner, S. (2007). Ethnic differences in lowdensity lipoprotein particle size in hypertensive adults. Journal of Clinical Lipidology, $1(3), 218-224$.

Lapidus, L., Bengtsson, C., Larsson, B., Pennert, K., Rybo, E., \& Sjostrom, L. (1984). Distribution of adipose tissue and risk of cardiovascular disease and death: A 12 year follow up of participants in the population study of women in Gothenburg, Sweden. British Medical Journal (Clinical Residence Edition), 289(6454), 1257-1261.

Larsson, B., Svardsudd, K., Welin, L., Wilhelmsen, L., Bjorntorp, P., \& Tibblin, G. (1984). Abdominal adipose tissue distribution, obesity, and risk of cardiovascular disease and death: 13 year follow up of participants in the study of men born in 1913. British Medical Journal (Clinical Residence Edition), 288(6428), 1401-1404.

LaVeist, T., Nickerson, K., \& Bowie, J. (2000). Attitudes about racism, medical mistrust, and satisfaction with care among African American and White cardiac patients. Medical Care Research and Review, 57, 146-161.

Lean, M., Han, T., \& Morrison, C. (1995). Waist circumference as a measure for indicating need for weight management. British Medical Journal, 311(6998), 158-161.

Lee, C. D., Blair, S. N., \& Jackson, A. S. (1999). Cardiorespiratory fitness, body composition, and all-cause and cardiovascular disease mortality in men. American Journal of Clinical Nutrition, 69(3), 373-380.

Libby, P., \& Ridker, P. (2004). Inflammation and atherosclerosis: Role of C-reactive protein in risk assessment. American Journal of Medicine, 116(6S1), 9-16.

Libby, P., Ridker, P., \& Maseri, A. (2002). Inflammation and atherosclerosis. Circulation, 105, 1135-1143.

LINCO Research. (2005). Human cardiovascular disease panel 1 LINCOplex kit (Catalog HCVD1-67AK). St. Charles, MO: Millipore. 
LoBiondo-Wood, G., \& Haber, J. (1990). Nursing research methods, critical appraisal, and utilization $\left(2^{\text {nd }}\right.$ ed.). St. Louis: Mosby.

Lutsey, P., Cushman, M., Steffen, L., Green D., Barr, R., Herrington, D., et al. (2006). Plasma hemostatic factors and endothelial markers in four racial/ethnic groups: The MESA Study. Journal of Thrombosis and Haemostasis, 4(12), 2629-2635.

Lynch, E., Liu, K., Kiefe, C., \& Greenland, P. (2006). Cardiovascular disease risk factor knowledge in young adults and 10-year change in risk factors: The Coronary Artery Risk Development in Young Adults (CARDIA) Study. American Journal of Epidemiology, 164(12), 1171.

Majka, D. S., Chang, R. W., Vu, T. T., Palmas, W., Geffken, D. F., Ouyang, P., Ni, H., \& Liu, K. (2009). Physical activity and high-sensitivity C-reactive protein: The MultiEthnic Study of Atherosclerosis. American Journal of Preventive Medicine, 36(1), 56-62.

Marrugat, J., Subirana, I., Comin, E., Cabezas, C., Vila, J., Elosua, R., et al. (2007). Validity of an adaptation of the Framingham cardiovascular risk function: The VERIFICA Study. Journal of Epidemiology \& Community Health, 61(1), 40-47.

Matsumoto, K., Sera, Y., Abe, Y., Tominaga, T., Horikami, K., Hirao, K. et al. (2002). High serum concentrations of soluble E-selectin correlate with obesity but not fat distribution in patients with type 2 diabetes mellitus. Metabolism, 51(7), 932-934.

McSweeney, J., Lefler, L., Fischer, E., Naylor Jr, A., \& Evans, L. (2007). CE Women's prehospital delay associated with myocardial infarction: does race really matter? Journal of Cardiovascular Nursing, 22(4), 279.

McTigue, K., Garrett, J., \& Popkin, B. (2002). The natural history of the development of obesity in a cohort of young US adults between 1981 and 1998. Annals of Internal Medicine, 136(12), 857-864.

Medscape, C., Connect, M., Care, C., Surgery, G., \& Today, M. (2005). The prevalence of the metabolic syndrome using the National Cholesterol Educational Program and International Diabetes Federation Definitions. Current Medical Research \& Opinion, 21(8), 1157-1159.

Meijer, G. A., Westerterp, K. R., Verhoeven, F. M., Koper, H. B., \& ten Hoor, F. (1991). Methods to assess physical activity with special reference to motion sensors and accelerometers. IEEE Transactions on Biomedical Engineering, 38(3), 221-229.

Mello, M., Studdert, D., \& Brennan, T. (2006). Obesity-The new frontier of public health law. New England Journal of Medicine, 354(24), 2601.

Mertens, I., \& Van Gaal, L. F. (2002). Obesity, haemostasis and the fibrinolytic system. Obesity Reviews, 3(2), 85-101. 
Molarius, A., \& Seidell, J. C. (1998). Selection of anthropometric indicators for classification of abdominal fatness - A critical review. International Journal of Obesity Related Metabolic Disorders, 22(8), 719-727.

Molarius, A., Seidell, J. C., Sans, S., Tuomilehto, J., \& Kuulasmaa, K. (2000). Educational level, relative weight, and changes in their association over 10 years: An international perspective from the WHO MONICA Project. American Journal of Public Health, 90(8), 1260-1268.

Mora, S., Lee, I., Buring, J., \& Ridker, P. (2006). Association of physical activity and body mass index with novel and traditional cardiovascular biomarkers in women. JAMA, 295(12), 1412-1419.

Mora, S., Rifai, N., Buring, J., \& Ridker, P. (2006). Additive value of immunoassaymeasured fibrinogen and high-sensitivity $\mathrm{C}$-reactive protein levels for predicting incident cardiovascular events. Circulation, 114(5), 381.

Mosca, L., Ferris, A., Fabunmi, R., \& Robertson, R. (2004). Tracking women's awareness of heart disease: An American Heart Association national Study. Circulation, 109, 573-579.

Nasir, K., Budoff, M., Wong, N., Scheuner, M., Herrington, D., Arnett, D., et al. (2007). Family history of premature coronary heart disease and coronary artery calcification: multi-ethnic study of atherosclerosis (MESA). Circulation, 116(6), 619.

National Cholesterol Education Program, Adult Treatment Panel III. (2002). Third report of the expert panel on detection, evaluation, and treatment on high blood cholesterol in adults (Adult Treatment Panel III). ATP III At-A-Glance: Quick Desk. Reference. Retrieved January 8, 2009 from http://www.nhlbi.nih.gov/guidelines/cholesterol/atglance.htm

National Heart Lung and Blood Institute. (2006). Classification of overweight and obesity by BMI, waist circumference, and associated disease risks. Retrieved February 16, 2009, from http://www.nhlbi.nih.gov/health/public/heart/obesity/lose_wt/bmi_dis.htm

National Institutes of Health, National Heart, Lung, and Blood Institute. (1998). Clinical guidelines on the identification, evaluation, and treatment of overweight and obesity in adults: The evidence report. Retrieved March 6, 2008, from http://www.nhlbi.nih.gov/guidelines/obesity/ob gdlns.htm

Nelson, T. L., Hunt, K. J., Rosamond, W. D., Ammerman, A. S., Keyserling, T. C., Mokdad, A. H. \& Will, J. C. (2002). Obesity and associated coronary heart disease risk factors in a population of low-income African American and White women: The North Carolina WISEWOMAN Project. Preventive Medicine, 35(1), 1-6. 
Nestle, M., \& Jacobson, M. (2000). Halting the obesity epidemic: A public health policy approach. Public Health Reports, 115(1), 12.

Nguyen, V. H., \& McLaughlin, M. A. (2002). Coronary artery disease in women: A review of emerging cardiovascular risk factors. Mt Sinai Journal of Medicine, 69(5), 338349.

Nijhuis, J., van Dielen, F. M. H., \& Fouraschen, S. M. G. (2007). Endothelial activation markers and their key regulators after restrictive bariatric surgery. Obesity Research, $15(6), 4$.

Ogden, C., Carroll, M., Curtin, L., McDowell, M., Tabak, C., \& Flegal, K. (2006). Prevalence of overweight and obesity in the United States, 1999-2004. JAMA, 295(13), 1549-1555.

Onusko, E. (2003). Diagnosing secondary hypertension. American Family Physician, $67(1), 67-74$.

Perry, A., Wang, X., Goldberg, R., Ross, R., \& Jackson, L. (2007). The relationship between cardiometabolic and hemostatic variables: Influence of race. Metabolism, 57(2), 200-206.

Philbin, E., McCullough, P., DiSalvo, T., Dec, G., Jenkins, P., \& Weaver, W. (2000). Socioeconomic status is an important determinant of the use of invasive procedures after acute myocardial infarction in New York State. Circulation, 102, 111-115.

Poirier, P., \& Eckel, R. (2002). Obesity and cardiovascular disease. Current Atherosclerosis Reports, 4(6), 448-453.

Pouliot, M. C., Despres, J. P., Lemieux, S., Moorjani, S., Bouchard, C., Tremblay, A., et al. (1994). Waist circumference and abdominal sagittal diameter: Best simple anthropometric indexes of abdominal visceral adipose tissue accumulation and related cardiovascular risk in men and women. American Journal of Cardiology, 73(7), 460-468.

Prineas, R., Folsom, A., \& Kaye, S. (1993). Central adiposity and increased risk of coronary artery disease mortality in older women. Annals of Epidemiology, 3(1), 35-41.

Qi, L., Hu, F. B., \& Hu, G. (2008). Genes, environment, and interactions in prevention of type 2 diabetes: A focus on physical activity and lifestyle changes. Current Molecular Medicine, 8(6), 519-532.

Rao, S., Donahue, M., Pi-Sunyer, F., \& Fuster, V. (2001). Obesity as a risk factor in coronary artery disease. American Heart Journal, 142(6), 1102. 
Rawson, E. S., Freedson, P. S., Osganian, S. K., Matthews, C. E., Reed, G., \& Ockene, I. S. (2003). Body mass index, but not physical activity, is associated with C-reactive protein. Medicine \& Science in Sports \& Exercise, 35(7), 1160-1166.

Richardson, M., Ainsworth, B., Jacobs, D., \& Leon, A. (2001). Validation of the Stanford 7-Day Recall to assess habitual physical activity. Annals of Epidemiology, 11(2), 145153.

Ridker, P., Hennekens, C., Buring, J., \& Rifai, N. (2000). C-reactive protein and other markers of inflammation in the prediction of cardiovascular disease in women. New England Journal of Medicine, 342(12), 836-843.

Rifai, N., Tracy, R. P., \& Ridker, P. M. (1999). Clinical efficacy of an automated highsensitivity C-reactive protein assay. Clinical Chemistry, 45(12), 2136-2141.

Rifai, N., \& Ridker, P. (2001). High-sensitivity C-reactive protein: A novel and promising marker of coronary heart disease. Clinical Chemistry, 47(3), 403-411.

Ridker, P., Danielson, E., Fonseca, F., Genest, J., Gotto Jr, A., Kastelein, J., et al. (2008). Rosuvastatin to prevent vascular events in men and women with elevated C-reactive protein. New England Journal of Medicine, 359(21), 2195.

Ridker, P., Stampfer, M., \& Rifai, N. (2001). Novel risk factors for systemic atherosclerosis: A comparison of C-reactive protein, fibrinogen, homocysteine, lipoprotein (a), and standard cholesterol screening as predictors of peripheral arterial disease. JAMA, 285(19), 2481-2485.

Ridker, P. M. (2008). High-sensitivity C-reactive protein as a predictor of all-cause mortality: implications for research and patient care. Clinical Chemistry, 54(2), 234-237.

Ridker, P. M., Hennekens, C. H., Buring, J. E., \& Rifai, N. (2000). C-reactive protein and other markers of inflammation in the prediction of cardiovascular disease in women. New England Journal of Medicine, 342(12), 836-843.

Rimm, E. B., Stampfer, M. J., Giovannucci, E., Ascherio, A., Spiegelman, D., Colditz, G. A., et al. (1995). Body size and fat distribution as predictors of coronary heart disease among middle-aged and older US men. American Journal of Epidemiology, 141(12), 1117-1127.

Ritz, E. (2003). Cardiovascular risk factors and urinary albumin: Vive la petite difference, American Society of Nephrology, 14, 1415-1416.

Rodriguez-Arcos, R. C., Smith, A. C., \& Waldron, K. W. (2002). Effect of storage on wall-bound phenolics in green asparagus. Journal of Agricultural \& Food Chemiatry, 50(11), 3197-3203. 
Rosenson, R. S., \& Koenig, W. (2003). Utility of inflammatory markers in the management of coronary artery disease. American Journal of Cardiology, 92(1A), 10i$18 \mathrm{i}$.

Ross, R. (1999). Atherosclerosis-An inflammatory disease. New England Journal of Medicine, 340(2), 115-126.

Rutter, M., Meigs, J., Sullivan, L., D'Agostino, R., \& Wilson, P. (2004). C-reactive protein, the metabolic syndrome, and prediction of cardiovascular events in the Framingham Offspring Study. Circulation, 110, 380-385.

Sallis, J. F., Haskell, W. L., Wood, P. D., Fortmann, S. P., Rogers, T., Blair, S. N., et al. (1985). Physical activity assessment methodology in the Five-City Project. American Journal of Epidemiology, 121(1), 91-106.

Santos, A. C., Lopes, C., Guimaraes, J. T., \& Barros, H. (2005). Central obesity as a major determinant of increased high-sensitivity $\mathrm{C}$-reactive protein in metabolic syndrome. International Journal of Obesity, 29(12), 1452-1456.

Scherrer, U., Nussberger, J., Torriani, S., Waeber, B., Darioli, R., Hofstetter, J. R., et al. (1991). Effect of weight reduction in moderately overweight patients on recorded ambulatory blood pressure and free cytosolic platelet calcium. Circulation, 83(2), 552558.

Schoeller, D. (1988). Measurement of energy expenditure in free-living humans by using doubly labeled water. Journal of Nutrition, 118(11), 1278.

Schoeller, D., Taylor, P., \& Shay, K. (1995). Analytic requirements for the doubly labeled water method. Obesity Research, 3(1), 15-20.

Schutte, R., Huisman, H., Schutte, A., \& Malan, N. (2005). Leptin is independently associated with systolic blood pressure, pulse pressure and arterial compliance in hypertensive African women with increased adiposity: The POWIRS Study. Journal of Human Hypertension, 19, 535-541.

Smith, A., Patterson, C., Yarnell, J., Rumley, A., Ben-Shlomo, Y., \& Lowe, G. (2005). Which hemostatic markers add to the predictive value of conventional risk factors for coronary heart disease and ischemic stroke? The Caerphilly Study. Circulation, 112, 3080-3087.

Snitker, S., Le, K. Y., Hager, E., Caballero, B., \& Black, M. M. (2007). Association of physical activity and body composition with insulin sensitivity in a community sample of adolescents. Archives of Pediatrics \& Adolescent Medicine, 161(7), 677-683. 
Solano, M. P., Perry, A. C., Wang, X., Ross, R., \& Goldberg, R. B. (2003). Insulin resistance but not visceral adipose tissue is associated with plasminogen activator inhibitor type 1 levels in overweight and obese premenopausal African American women. International Journal of Obesity, 27, 82-87.

Srinivasan, S., Bao, W., Wattigney, W., \& Berenson, G. (1996). Adolescent overweight is associated with adult overweight and related multiple cardiovascular risk factors: The Bogalusa Heart Study. Metabolism, 45(2), 235-240.

Staffileno, B. A., Minnick, A., Coke, L. A., \& Hollenberg, S. M. (2007). Blood pressure responses to lifestyle physical activity among young, hypertension-prone AfricanAmerican women. Journal of Cardiovascular Nursing, 22(2), 107-117.

Stevens, J., Cai, J., Evenson, K., \& Thomas, R. (2002). Fitness and fatness as predictors of mortality from all causes and from cardiovascular disease in men and women in the Lipid Research Clinics Study. American Journal of Epidemiology, 156(9), 832.

Suka, M., Sugimori, H., \& Yoshida, K. (2002). Validity of the Framingham risk model applied to Japanese men. Methods of Information in Medicine, 41(3), 213-215.

Sumner, A. E., Sen, S., Ricks, M., Frempong, B. A., Sebring, N. G., \& Kushner, H. (2008). Determining the waist circumference in African Americans which best predicts insulin resistance. Obesity (Silver Spring), 16(4), 841-846.

Taeye, B., Smith, L., \& Vaughan, D. (2005). Plasminogen activator inhibitor-1: A common denominator in obesity, diabetes and cardiovascular disease. Current Opinion in Pharmacology, 5(2), 149-154.

Taylor, H., Liu, J., Wilson, G., Golden, S. H., Crook, E., Brunson, C. D., Steffes, M., Johnson, W. D., \& Sung, J. H. (2008). Distinct component profiles and high risk among African Americans with metabolic syndrome: The Jackson Heart Study. Diabetes Care, 31(6), 1248-1253.

Tran, Z. V., \& Weltman, A. (1985). Differential effects of exercise on serum lipid and lipoprotein levels seen with changes in body weight. A meta-analysis. JAMA, 254(7), 919-924.

Tudor-Locke, C. E., \& Myers, A. M. (2001). Challenges and opportunities for measuring physical activity in sedentary adults. Sports Medicine, 31(2), 91-100.

Tuomilehto, J., Lindstrom, J., Eriksson, J. G., Valle, T. T., Hamalainen, H., IlanneParikka, P., et al. (2001). Prevention of type 2 diabetes mellitus by changes in lifestyle among subjects with impaired glucose tolerance. New England Journal of Medicine, 344(18), 1343-1350. 
United States Department of Health and Human Services (2000). Healthy people 2010. $2^{\text {nd }}$ ed. with understanding and improving health and objectives for improving health. Washington, DC: U.S. government printing office.

United States Department of Health and Human Services. (2002). Physical activity fundamental to preventing disease. Retrieved February 16, 2009, from http://aspe.hhs.gov/health/reports/physicalactivity/

Vazquez, G., Duval, S., Jacobs Jr, D., \& Silventoinen, K. (2007). Comparison of body mass index, waist circumference, and waist/hip ratio in predicting incident diabetes: A meta-analysis. Epidemiologic Reviews, 29(1), 115.

Velasquez-Mieyer, P. A., Christensen, M. L., Lustig, R. H., Buffington, C. K., Sinclair, J., Rose, S. R., et al. (2000). Role of race and obesity in the pathogenesis of type 2 diabetes mellitus in children. Paper presented at the International Congress of Endocrinology, Australia.

Velasquez-Mieyer, P. A., Cowan, P. A., Neira, C. P., \& Tylavsky, F. (2008). Assessing the risk of impaired glucose metabolism in overweight adolescents in a clinical setting. Journal of Nutrition, Health \& Aging, 12(10), 750s-757s.

Visser, G., Boon, P., \& Meijer, H. (2000). Validation of the doubly labeled water method in Japanese Quail Coturnix c. japonica chicks: Is there an effect of growth rate? Journal of Comparative Physiology B: Biochemical, Systemic, and Environmental Physiology, 170(5), 365-372.

Walker, K. Z., Piers, L. S., Putt, R. S., Jones, J. A., \& O'Dea, K. (1999). Effects of regular walking on cardiovascular risk factors and body composition in normoglycemic women and women with type 2 diabetes. Diabetes Care, 22(4), 555-561.

Washburn, R. A., Jacobsen, D. J., Sonko, B. J., Hill, J. O., \& Donnelly, J. E. (2003). The validity of the Stanford Seven-Day Physical Activity Recall in young adults. Medicine \& Science in Sports \& Exercise, 35(8), 1374-1380.

Wee, C. C., Girotra, S., Weinstein, A. R., Mittleman, M. A., Mukamal, K. J. (2008). The relationship between obesity and atherosclerotic progression and prognosis among patients with coronary artery bypass grafts: The effect of aggressive statin therapy. Journal of American College of Cardiology, 52, 620-625.

Westerterp, K. R. (1999). Physical activity assessment with accelerometers. International Journal of Obesity Related Metabolic Disorders, 23 Suppl 3, S45-49.

Wood, P. D., Stefanick, M. L., Williams, P. T., \& Haskell, W. L. (1991). The effects on plasma lipoproteins of a prudent weight-reducing diet, with or without exercise, in overweight men and women. New England Journal of Medicine, 325(7), 461-466. 
World Health Organization (2000). Obesity: Preventing and managing the global epidemic. Retrieved November 17, 2008, from

http://www.phpc.cam.ac.uk/mst/phtop/t3/whoobes.pdf

World Health Organization. (2004). Diet and physical activity. Retrieved August 19, 2008, from http://www.who.int/dietphysicalactivity/en/

Wilcox, A. J., Baird, D. D., Dunson, D., McChesney, R., \& Weinberg, C. R. (2001). Natural limits of pregnancy testing in relation to the expected menstrual period. JAMA, 286(14), 1759-1761.

Wilson, P., D'Agostino, R., Levy, D., Belanger, A., Silbershatz, H., \& Kannel, W. (1998). Prediction of coronary heart disease using risk factor categories. Circulation, 97, 18371847.

Wilson, P., D'Agostino, R., Sullivan, L., Parise, H., \& Kannel, W. (2002). Overweight and obesity as determinants of cardiovascular risk: The Framingham experience. Archives of Internal Medicine, 162(16), 1867-1872.

Wilson, P. W., D'Agostino, R. B., Levy, D., Belanger, A. M., Silbershatz, H., \& Kannel, W. B. (1998). Prediction of coronary heart disease using risk factor categories.

Circulation, 97(18), 1837-1847.

Winkleby, M., Kraemer, H., Ahn, D., \& Varady, A. (1998). Ethnic and socioeconomic differences in cardiovascular disease risk factors: Findings for women From the Third National Health and Nutrition Examination Survey, 1988-1994. JAMA, 280(4), 356-362.

Wong, T., Webster, J., Montoye, H., \& Washburn, R. (1981). Portable accelerometer device for measuring human energy expenditure. IEEE Transactions on Biomedical Engineering, 28(6), 467-471.

Wu, T., McGrath, K., \& Death, A. (2005). Cardiovascular disease in diabetic nephropathy patients: Cell adhesion molecules as potential markers? Vascular Health and Risk Management, 1(4), 309.

Yanovski, J., Diament, A., Sovik, K., Nguyen, T., Li, H., Sebring, N., et al. (2000). Associations between uncoupling protein 2, body composition, and resting energy expenditure in lean and obese African American, white, and Asian children. American Journal of Clinical Nutrition, 71(6), 1405.

Yudkin, J., Stehouwer, C., Emeis, J., \& Coppack, S. (1999). C-reactive protein in healthy subjects: Associations with obesity, insulin resistance, and endothelial dysfunction a potential role for cytokines originating from adipose tissue? Arteriosclerosis, Thrombosis, and Vascular Biology, 19, 972-978. 
Zhang, X., Shu, X., Signorello, L., Hargreaves, M., Cai, Q., Linton, M., et al. (2008). Correlates of high serum C-reactive protein levels in a socioeconomically disadvantaged population. Disease Markers, 24(6), 351-359. 


\section{APPENDIX A. DEMOGRAPHIC QUESTIONNAIRE}

\section{Demograne}

Participant ID:

Please circle the answer that applies to you where indicated or fill in the correct answers

What is your gender?

* Female

s Male

* Other

What is your age?

What is your marital status?

- Single, never married,

Married

s Separated,

ta Divorced,

- Widowed,

* Other

How many people, including yourself, are in your household?

What is the highest level of education you have completed?

* Grade School,

s Some High School,

:High School or GED,

as 2-year College,

- 4-year College

Graduate School

What is your employment status?

s Part-time,

as Full-time,

- Full-time homemaker

- College/University Student,

- Self-employed,

"Retired,

N Not Employed

What was your household's total annual income for the most recent calendar year?

* Less than $\$ 25,000$

- $\$ 25,000-\$ 49,999$

a $\$ 50,000-\$ 74,999$

- $\$ 75,000-\$ 99,999$

. $\$ 100,000$ or more

RE APPQOULDATE44.05 EPIMATONDATE $W H$

RE th 390 APFROVD BV: 14 
When was your last menstrual period?

Have you had a hysterectomy?

* Yes

No

Are you taking hormone replacement therapy?

- Yes

No

Have you ever used tobacco/cigarette/snuff/pipe (Circle all that apply)

- Yes

- No

If you have used tobacco/cigarette/snuff/pipe, how much per day

Do you still use tobacco/cigarette/snuff/pipe?

ॠ Yes

No

Do you drink alcohol?

* Yes

- No

If you drink aicohol, how much do you drink

Have you ever used marijuana/speed/cocaine (Circle all that apply)

- Yes

No

If you have used marijuana/speed/cocaine, how much did you use per week

Do you still use marijuana/speed/cocaine?

a Yes

No

Do you have or have you ever had high blood pressure and/or diabetes?

- Yes

* No

Are you currently on medications for high blood pressure or diabetes?

- Yes

- No

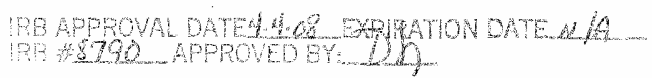




\section{APPENDIX B. 7-DAY PHYSICAL ACTIVITY RECALL QUESTIONNAIRE}

\section{7-Day Physical Activity Recall Questionanare}

Day of the week form completed:

1. Were you employed in the last seven days? Yes No

2. How many days of the last seven did you work? (round to nearest day)

3. How many total hours did you work in the last seven days? hours

4. What days of the week do you consider to be your weekend or non-work days? For most people this would be Saturday and Sunday but it may be different for you._Sunday_ Monday_ Tuesday _ Wednesday_Thursday_Friday _ Saturday?

5. If you did not work your usual week, why did you work less than usual?

6. For the past seven days, and thinking only about activities that are at least of moderate intensity, how many days did you do activity or exercise that added up to at least 30 minutes each day? number of days $(0$ to 7$)$

7. Was this a typical week in terms of your usual pattern of activity or exercise? Yes No; lf No were you more or less active in the past week than you usually are? More Less

Until now, we've just been talking about the last seven days. Now I would like you to think about your usual activities over the last three months.

8. During your work week, on average how many hours per day do you spend sitting Quietly (e.g. watching TV, working at a desk or computer, eating, or reading)? average hours per day.

During your weekend, on average how many hours per day do you spend sitting quietly (e.g. watching TV, working at a desk or computer, eating, or reading)? average hours per day.

9. How many flights of stairs do you climb up each day? ( 1 flight $=10$ steps $)$ number of flights. 
10. If you had to add together the total minutes you spend walking during the day, how many minutes would that be? Remember, add up your actual walking time and don't add in the time spent just standing include your to and from walking and any fitness walking. Don't try to remember every step, just give a general idea of the time spent walking. total minutes per day.

11. What is your usual pace of walking? Mark ONE only.

_Casual or strolling (less than 2 miles per hour)

_. Average or normal (2 to 3 miles per hour)

_. Fairly brisk ( 3 to 4 miles per hour)

_Brisk or striding ( 4 miles per hour or faster)

12. Do you regularly do strength and flexibility exercises like sit-ups, push-ups, yoga, or stretching?

_ Yes, if yes, How many days per week do you do these exercises? _ _ number of days

- No

13. On the days that you do strength and flexibility exercises, how many minutes do you spend doing them? total minutes. 
Yesterday

\begin{tabular}{|l|l|l|l|l|l|l|l|l|}
\hline & $\begin{array}{l}\text { Days of } \\
\text { the week }\end{array}$ & HRS MN & HRS MIN & HRS MIN & HRS MN & HRS MN & HRS MN & HRS MN \\
\hline & Sleep & & & & & & & \\
\hline Morning & Moderate & & & & & & & \\
\hline & Hard & & & & & & & \\
\hline & Very Hard & & & & & & & \\
\hline Afternoon & Moderate & & & & & & & \\
\hline & Hard & & & & & & & \\
\hline Evening & Mederate & & & & & & & \\
\hline & Hard & & & & & & & \\
\hline & Very Hard & & & & & & & \\
\hline
\end{tabular}

Calculated Energy Expenditure $\mathrm{Kcal} / \mathrm{kg} /$ day 


\title{
APPENDIX C. UNIVERSITY OF TENNESSEE HEALTH SCIENCE CENTER INSTITUTIONAL REVIEW BOARD APPROVAL
}

\author{
THEUNIVERSITYOF \\ IENNESSEE WE \\ HEALTH SCIENCE CENTER \\ April 4, 2008 \\ Institutional Reviaw Borrd \\ 910 Madison Avenue, Suite 600 \\ Memphis, TN 38163 \\ Queen Henry-Okafor \\ Phone: (901) $448-4824$ \\ College of Graduate Health Sciences \\ Department of Nursing \\ 920 Madison \\ Suite 507 \\ UTHSC \\ CAMPUS
}

RE: "The Impact of Obesity on the Traditional and Emerging Cardiovascular Disease Risk Factors in African American Women" (IRB \#8790)

Dear Ms. Henry-Okafor,

We are in receipt of your written acceptance dated April 3, 2008 in response to our communication dated April 3, 2008 concerning the above referenced Institutional Review Board Project.

The Administrative Section of the UTHSC Institutional Review Board (IRB) determined your application to be consistent with the guidelines for expedited review under categories (2), (3), (4) and (7). Therefore, this application was approved in this regard as complying with proper consideration of the rights and welfare of human subjects, the risk involved, and the potential benefits of the study. This letter constitutes full approval of your application, questionnaires, flyers and consent form, stamped approved by the UTHSC IRB on April 4, 2008 for the above referenced study. This project was approved for 12 months with an expiration date of April 4, 2009. The consent form dated April 3, 2008 is also approved from the date of this letter through April $4,2009$.

The IRB has also determined that the informed consent form, incorporating the authorization of subjects to use their protected health information in research, complies with the federal privacy regulations as specified in 45 CFR160 and 45 CFR 164.

In the event that subjects are to be recruited using solicitation materials, such as brochures, posters, website based advertisement, etc., these materials must receive prior approval of the IRB. Any revisions in the approved application must also be submitted to and approved by the IRB prior to implementation. In addition, you are responsible for reporting any unanticipated serious adverse events or other problems involving risks to subjecis or others in the manner required by the local IRB policy.

Finally, reapproval of your project is required by the IRB in accord with the conditions specified above. You may not continue beyond the time or other limits specified unless you obtain prior written approval of the IRB.
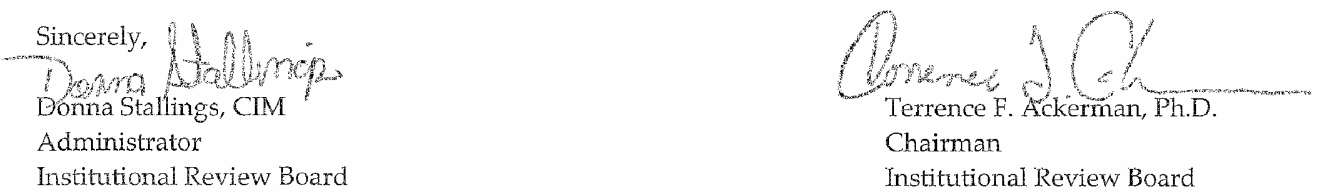
Preparation date 3/28/08

\title{
Join Us for Free Heart
}

Healthy Breakfast

\author{
$\&$
}

Screening for Heart Disease

WHY: A research study on "The Impact of body weight on the traditionallemerging risk factors for heart disease in African American Women".

WHO: African American Women ages 18. 45 are invited to take part.

WHERE: HELP Center 920 Madison Avenue, Suite 507, UTMEM. I visit only lasting for about an hour.

COSTS/BENEFITS: No cost to participate. You will receive payment in addition to a heart healthy breakfast.

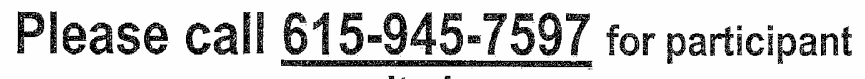
criteria. 


\section{Research Study examining}

\section{The Impact of Body Weight on the Traditional and Emerging Cardiovascular Disease Risk Factors in African American Women (18-45 year olds)}

The purpose of this research study is to explore the relationship between body weight and the traditional and newer risk factors for heart disease.

- Free screening for risk factors for heart disease.

- One time visit lasting about 1-2 hours

- Participants will receive free heart healthy breakfast

- Plus cash gift

To learn more about the study, call

Queen Henry-Okafor, PhD candidate, RN

615-945-7597 (cell phone)

Tear off and call for more info:

Queen Henry-Okafor, PhD Candidate, RN

Heart disease risk factor study

615-945-7597

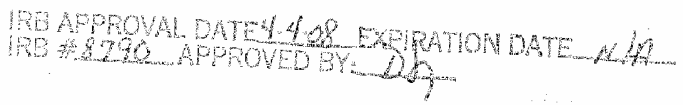




\title{
APPENDIX E. CONSENT FORM
}

Cardiac Risk Factors; PI: Queen Henry-Okafor

\author{
University of Tennessee Health Science Center
}

Consent to Participate in a Research Study

\section{CONSENT FORM}

The Impact of Obesity on the Traditional and Emerging Cardiovascular Disease Risk Factors in African American Women

\author{
Principal Investigator: Queen O. Henry-Okafor, PhD(c), APRN \\ University of Tennessee Health Science Center \\ 920 Madison Avenue, Suite 507 \\ Memphis, TN 38163 \\ $615-945-7597$ \\ Co-Investigators: Patricia A. Cowan, PhD \\ Mona N. Wicks, PhD \\ Margaret (Carrie) Harvey, PhD, APRN
}

\section{MTRODUCTION:}

You are being given the opportunity to participate in a research study on "The Impact of Obesity on the Traditional and Emerging Cardiovascular Disease Risk Factors in African American Women". This study is being conducted at the University of Tennessee Health Science Center Memphis and a Family Practice Clinic in Nashville. The Principal Investigator, Queen Henry-Okafor, is a candidate for a doctoral degree. There will be about 64 healthy African American women who are between 18 to 45 years old will participate in this study. You will take part in the study for one visit that will last for about one to two hours. The entire study will be completed in 6 months.

This study is being done to help understand the relationship between body weight and the levels of some traditional (known) and emerging (possible, newer) cardiovascular disease (heart disease) risk factors. Traditional cardiovascular disease risk factors include high blood pressure, high cholesterol levels (fat in the blood), abnormal blood sugar, physical inactivity, and obesity (fatness). We are concerned because traditional cardiovascular disease risk factors that have been used to predict the risk of cardiovascular disease do not seem to adequately explain the severity of cardiovascular disease seen in African American women. Recent studies suggest that inflammatory (substances in the blood that suggest swelling when there is no swelling) and thrombotic (substances in the blood that are involved in blood clots) markers could be useful in determining who is at risk for developing cardiovascular disease in otherwise healthy persons. Knowledge of the levels of these substances will help to identify significant risk of cardiovascular disease and further guide treatment and management procedures. If you agree to participate in this study, you will be asked to read and sign a copy of this informed consent. 
2. PROCEDURES TO BE FOLLOWED:

The research procedures are as follows:

- Urime Pregnancy Test

You will be asked to urinate into a plastic container so that a urine pregnancy test can be done. If the pregnancy test is positive, you will not be able to participate in the study or receive any money. You will be referred to your primary healthcare provider for care.

- Anthropometric Assessment

You will have your height, weight, waist, and hip measured.

- Blood Pressure Assessment

You will sit for 15 minutes in a chair and your blood pressure will be taken twice.

- Demographic Questionnaire

You will complete a questionnaire that ask questions about age, marital status, income, social, medical, and medication history.

- Physical Activity Questionnaire

You will complete a questionnaire and answer questions about your physical activities during the past seven days.

- You will be given written results of your pregnancy test, anthropometric assessments (body weight, height, waist, and hip measurements), and blood pressure measurements at the end of the testing. You will provide a mailing address with a stamped envelop that has your assigned participant code where your laboratory results will be sent to you when available. You accept responsibility to take these results to your primary care provider for further review.

- Traditional/emerging cardiovascular risk factors

About $30 \mathrm{cc}$ (or about 2 tablespoons) of blood will be drawn from a vein in your arm. This will be used to measure your blood sugar, and other traditional and newer risk factors for cardiovascular disease. Your specimens will be coded to store them anonymously. No personal or identifiable information will be disclosed.

3. BENEFITS ASSOCIATED WITH PARTICIPATION:

The benefits to you for participation in this study may include:

- Early detection of cardiovascular risk factors such as diabetes, high cholestesterol (fat in the blood), or heart problems, which may lead to early referral, or treatment of such conditions. However, you may not receive any benefit from participating in the study.

- Society may benefit from this study because study results could provide information that would help doctors and nurses learn more about traditional and newer risk factors for cardiovascular disease in African American women and how obesity affects these risk factors. This study is not designed to treat any illness or to improve your health. However, your participation will enhance health-related knowledge about the early detection of cardiovascular disease. 


\section{RISKS ASSOCIATED WITH PARTICIPATION:}

There will be minimail risks associated with participation in the study which includes:

- There is a potential psychological risk if a pregnancy test turns out to be positive. You will be informed if the pregnancy test is positive and will be advised to follow up with your Primary Care Provider for appropriate counseling.

- There is also a potential psychological risk if previously unknown health care conditions are identified as a result of participating in this study. You will be notified of those findings and advised to follow up with your Primary Care Provider.

- You may experience feelings of thirst or hunger as a result of not eating or drinking during the 10 hours prior to the festing. You will be offered a free heart healthy breakfast after the testing. There is no health risk associated with collecting information from the questionnaires.

- Risks associated with having your blood drawn include the possibility of pain, bruising, bleeding or infection at the site of your blood draw. There is also the possibijity of a psychological distress if the venipuncture is not successful on the first attempt and a second attempt is needed. A warm heating pad will be applied to make it easier to find the vein. Finger pressure and/or ice packs will be applied for at least 5 minutes after blood drawing, if needed to minimize bleeding and the risk of bruising. A band-aid will be applied to the venipuncture site to prevent the possibility of infection.

\section{ALTERNATIVES TOPARTICIPATION:}

As an alterative to participating in the study, you may chose to have these tests performed by your health care provider or chose not to participate in the study.

\section{CONFIDENTIALITY:}

You have the right to privacy, and all information obtained in this study that identifies you will remain confidential (private) according to state and federal laws. Your research records will be labeled with a code number. A master key that links your name and the code number will be kept in a separate and secure location by the principal investigator. Access to the stored research results and your identifiers will be limited only to research personnel. The research results for you and all research subjects will not be in any medical records and other third parties (such as employers or insurers) will not have access to the research results. Only the principal investigator and/or co-investigators, local, state and/or federal governmental agencies, and the University of Tennessee Health Science Institutional Review Board will have access to confidential information collected in the study. This is necessary to assure that the records are correct and that your rights and welfare are adequately protected. If you sign this consent form, you are giving permission for the release of information from your medical records to the principal investigator and/or co-investigators and any of these parties. Your name will not be revealed in any reports or publications resulting from this study.

Under federal privacy regulations, you have the right to determine who has access to your personal health information (called "protected health information" or PHI). PHI collected in this study may include your medical history, the results of physical exams, lab tests, $x$-ray exams, and other diagnostic and treatment procedures. Basic information about you, such as your age, race, gender, or other similar information may be collected and is considered PHI. By signing this consent form, you are authorizing the researchers at the University of TN Health Science Center and UT Cancer Institute to have access to your PHI collected in this 
Cardiac Risk Factors; PI: Queen Henry-Okafor

study and to receive your PHI from your physician and/or facilities where you have received health care. In addition, your PHI may be shared with other persons involved in the conduct or oversight of this research, such as researchers at the University of Tennessee Health Science Center.

The Institutional Review Board (RB) at the University of TN Health Science Center may review your PHI as part of its responsibility to protect the rights and wellare of research subjects. Your PHI will not be used or disclosed to any other person or entity, except as required by law, or for authorized oversight of this research study by other regulatory agencies, or for other research for which the use and disclosure of your PHI has been approved by the IRB. Your PHI will be used only for the research purposes described in the intraduction of this consent form. Your PHI will be used until the study is completed.

You may cancel this authorization in writing at any time by contacting the principal investigator listed on the first page of the consent form. If you cancel the authorization, continued use of your PHI is permitted if it was obtained before the cancellation and its use is necessary in completing the research. However, PHI collected after your cancellation may not be used in the study. If you refuse to provide this authorization, you will not be able to participate in the research study. If you cancel the authorization, then you will be withdrawn from the study. Finally, the federal regulations allow you to obtain access to your PHI collected or used in this study.

\section{COMPENSATION AND TREATMENT FOR INUURY:}

I understand that $I$ am not waiving any legal rights or releasing the University of Tennessee or its agents from. liability for nogligence. I understand that, in the event of physical injury resulting from research procedures, the University of Temnessee does not have funds budgeted for compensation either for lost wages or for medical treatment. Therefore, the University of Tennessee does not provide for treatment or reimbursement for such injuries.

Treatment will be made available, including first aid, emergency treatment, and follow-up care as needcd. In the event of physical injury resulting from the research procedures, I or my insurance carrier will be billed for the costs associated with the medical treatment of a research related injury.

\section{QUESTIONS:}

You may contact the investigator at (615) 945-7597 to answer questions about the research study. In the event of a research related injury, you may contact the investigator at (615) 945-7597. You may contact Terrence $F$. Ackerman, UTHSC MRB Chairman at (901) $448-4824$ if you have any questions about your rights as a participant in this study or your rights as a research subject.

\section{PAYMENT FOR PARTICIPATION:}

You will be reimbursed for your time used to participate in this study. You will receive $\$ 20$ cash and a heart healthy breakfast after you complete all of the study tests.

\section{COSTS OF PARTICIPATION:}

There is no cost to you for participating in this study.

\section{PREMATURE TERMINATION:}

Given that the study will be a one-time contact with you, there will be no likelihood of termination once you are enrolled. You will be excluded from participation if you meet the following exclusion criteria: pregnancy, 
Cardiac Risk Factors; PI: Queen Henry-Okafor

University of Tennessee Health Science Center

Consent to Participate in a Research Study

\section{CONSENT FORM}

The Impact of Obesity on the Traditional and Emerging Cardiovascular Disease Risk Factors in African American Women

$\begin{array}{ll}\text { Principal Investigator: } & \text { Queen O. Henry-Okafor, PhD(c), APRN } \\ & \text { University of Tennessee Health Science Center } \\ & 920 \text { Madison Avenue, Suite 507 } \\ & \text { Memphis, TN } 38163 \\ & 615-945-7597 \\ & \\ \text { Co-Investigators: } & \text { Patricia A. Cowan, PhD } \\ & \text { Mona N. Wicks, PhD } \\ & \text { Margaret (Carrie) Harvey, PhD, APRN }\end{array}$

\section{INTRODUCTION:}

You are being given the opportunity to participate in a research study on "The Impact of Obesity on the Traditional and Emerging Cardiovascular Disease Risk Factors in African American Women". This study is being conducted at the University of Tennessee Health Science Center Memphis and a Family Practice Clinic in Nashville. The Principal Investigator, Queen Henry-Okafor, is a candidate for a doctoral degree. There will be about 64 healthy African American women who are between 18 to 45 years old will participate in this study. You will take part in the study for one visit that will last for about one to two hours. The entire study will be completed in 6 months.

This study is being done to help understand the relationship between body weight and the levels of some traditional (known) and emerging (possible, newer) cardiovascular disease (heart disease) risk factors. Traditional cardiovascular disease risk factors include high blood pressure, high cholesterol levels (fat in the blood), abnormal blood sugar, physical inactivity, and obesity (fatness). We are concerned because traditional cardiovascular disease risk factors that have been used to predict the risk of cardiovascular disease do not seem to adequately explain the severity of cardiovascular disease seen in African American women. Recent studies suggest that inflammatory (substances in the blood that suggest swelling when there is no swelling) and thrombotic (substances in the blood that are involved in blood clots) markers could be useful in determining who is at risk for developing cardiovascular disease in otherwise healthy persons. Knowledge of the levels of these substances will help to identify significant risk of cardiovascular disease and further guide treatment and management procedures. If you agree to participate in this study, you will be asked to read and sign a copy of this informed consent. 


\section{VITA}

Queen Obiageli Henry-Okafor earned a Bachelor's Degree in Mass

Communication from the University of Nigeria Nsukka in 1984 and an Associate Degree in Nursing from San Jacinto College Pasadena Texas in 1998. She later attended Tennessee State University in Nashville Tennessee from where she earned a Bachelor's Degree in Nursing and Master's Degree in Nursing Science as a family nurse practitioner in 2003. Her thesis explored the lived experience of African American women and spirituality. Queen has worked as a registered nurse in medical-surgical and psychiatric nursing as well as in prison health. As a nurse practitioner, she began her career in a family practice clinic and later in a Community clinic affiliated with Vanderbilt University School of Nursing's education and research programs. She later resigned her appointment to focus on family and doctoral studies. Queen's interest in translational research motivated her to pursue doctoral education in Nursing from the University of Tennessee Health Science Center in Memphis since 2005. Her research interest has been in the area of preventive cardiology and minority populations. She is a recipient of the Southern Regional Education Board Dissertation Year Fellowship Award. Her research advisor/mentor was Dr Patricia Cowan, who provided her with the expertise and guidance through this successful doctoral education. Queen plans to pursue a career in nursing education and research. 